\title{
Ultra-Low-Power Analog-to-Digital Converters for Medical Applications
}

Dai Zhang

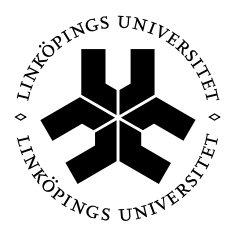

Linköping University
INSTITUTE OF TECHNOLocr

Division of Electronic Devices

Department of Electrical Engineering

Linköping University

SE-581 83 Linköping, Sweden

Linköping 2014 
Ultra-Low-Power Analog-to-Digital Converters for Medical Applications Copyright () 2014 Dai Zhang

ISBN 978-91-7519-264-2

ISSN 0345-7524

Printed by LiU-Tryck, Linköping, Sweden, 2014 


\section{Abstract}

Biomedical systems are commonly attached to or implanted into human bodies, and powered by harvested energy or small batteries. In these systems, analog-to-digital converters(ADCs) are key components as the interface between the analog world and the digital domain. Conversion of the low frequency bioelectric signals does not require high speed, but ultra-low-power operation. This combined with the required conversion accuracy makes the design of such ADCs a major challenge. Among prevalent ADC architectures, the successive-approximation-register (SAR) ADC exhibits significantly high energy efficiency due to its good trade-offs among power consumption, conversion accuracy, and design complexity. This thesis examines the physical limitations and investigates the design methodologies and circuit techniques for low-speed and ultra-low-power SAR ADCs.

The power consumption of SAR ADC is analyzed and its lower bounds are formulated in the thesis. At low resolutions, power is bounded by minimum feature sizes; while at medium to high resolutions, power is bounded by thermal noise and capacitor mismatch. In order to relax the mismatch requirement on the capacitor sizing while still ensuring enough linearity for high resolution, a bottom-up weight calibration technique is further proposed. It utilizes redundancy generated by a non-binary-weighted capacitive network, and measures the actual weights of more significant capacitors using less significant capacitors.

Three SAR ADCs have been implemented. The first ADC, fabricated in a $0.13 \mu \mathrm{m}$ CMOS process, achieves $9.1 \mathrm{ENOB}$ with $53-\mathrm{nW}$ power consumption at $1 \mathrm{kS} / \mathrm{s}$. The main key to achieve the ultra-low-power operation turns out to be the maximal simplicity in the ADC architecture and low transistor count. In addition, a dual-supply voltage scheme allows the SAR digital logic to operate at $0.4 \mathrm{~V}$, reducing the overall power consumption of the ADC by $15 \%$ without any loss in performance. Based on the understanding from the first ADC and motivated by the predicted power bounds, the second ADC, a single-supply 9.1-ENOB SAR ADC in 65nm CMOS process has been further fabricated. It achieves a substantial (94\%) improvement in power consumption with $3-\mathrm{nW}$ total power at $1 \mathrm{kS} / \mathrm{s}$ and $0.7 \mathrm{~V}$. Following the same concept of imposing maximal simplicity in the ADC architecture and taking advantage of the smaller feature size, the ultra-low-power consumption is achieved by a matched split- 
array capacitive DAC, a bottom-plate full-range input-sampling scheme, a latch-based SAR control logic, and a multi- $\mathrm{V}_{\mathrm{T}}$ design approach. The third ADC fabricated in $65 \mathrm{~nm}$ CMOS process targets at a higher resolution of $14 \mathrm{~b}$ and a wider bandwidth of $5 \mathrm{KHz}$. It achieves $12.5 \mathrm{ENOB}$ with $1.98-\mu \mathrm{W}$ power consumption at $0.8 \mathrm{~V}$ and $10 \mathrm{kS} / \mathrm{s}$. To achieve the high resolution, the ADC implements a uniform-geometry non-binaryweighted capacitive DAC and employs a secondary-bit approach to dynamically shift decision levels for error correction. Moreover, a comparator with bias control utilizes the redundancy to reduce the power consumption. 


\section{Populärvetenskaplig sammanfattning}

Biomedicinska system driver utvecklingen av hälsovården framåt. Sådana system möjliggör hantering av kroniska sjukdomar, medicinsk diagnos, och övervakning av välmåendet utanför traditionella kliniska miljöer, vilket ger högre livskvalitet till en lägre kostnad. Eftersom dessa system vanligtvis är monterade eller implanterade i människokroppen och drivs av skördad energi eller små batterier, så blir miniatyrisering och ultralåg effektförbrukning kritiska design specifikationer.

I dessa system, är analog-till-digital omvandlare (ADC:er) nyckelkomponenter som gränssnittet mellan den analoga världen och den digitala domänen. Omvandling av lågfrekventa bioelektriska signaler kräver inte hög fart, men ultralåg effektförbrukning. Detta kombinerat med den nödvändiga konverterings precisionen gör konstruktionen av sådana ADC:er till en stor utmaning. Denna avhandling utforskar de fysikaliska begränsningarna samt undersöker design metodiker och kretstekniker för låghastighets och ultralåg effekts ADC:er.

Bland olika ADC arkitekturer, så ger successiv approximations register (SAR) ADC:n på tagligt hög energieffektivitet p.g.a. dess balans mellan effektförbrukning, precision, och design komplexitet. Effektförbrukningen av SAR ADC:er är analyserad och dess lägre gräns är formulerad. Baserat på förståelsen utifrån analysen, implementeras tre SAR ADC:er. De två första använder maximal enkelhet i ADC arkitekturen för att nå ultralåg effektförbrukning vid moderata upplösningar. Den tredje använder redundans och digital kalibrering för att nå hög upplösning och använder samtidigt bias kontroll tekniker för att reducera effektförbrukningen. De tre ADC:erna fortsätter närma sig den teoretiska gränsen. 


\section{Acknowledgments}

Many people have supported and encouraged me during my years as a $\mathrm{PhD}$ student. I would like to express my deepest gratitude to them:

- my supervisor, Professor Atila Alvandpour, for offering me the opportunity to pursue my postgraduate study here. He is extremely supportive and has given me invaluable advice and help on doing research and paper writing.

- Professor emeritus Christer Svensson. Discussing research problems with him is very enjoyable. I hope that I have learned something out of his unique analytical approach. Christer is still active in research, being a great role model that encourages us to stay hungry and stay foolish.

- Christer Jansson for all the interesting discussions and invaluable comments on circuit design.

- Ameya Bhide for the great collaboration on the first chip. I am also grateful to him for being a friendly company. Life as a PhD student is full of struggling and self-questioning. Then it's good to know you are not alone :)

- Prakash Harikumar. He is a live dictionary. I benefit a lot from both technical and non-technical discussions with him. Oh, I almost forgot to say: Prakash, please don't work tooo much!

- Arta Alvandpour for designing the PCBs of the first two chips, solving toolrelated problems, and borrowing test equipments from companies.

- Timmy Sundström and Jonas Fritzin for their valuable help in research. Special thanks to Jonas for all the help and encouraging words during my job search.

- Zhenzhi Wu for helping me to estimate the hardware cost of the error correction.

- Martin Nielsen Lönn for helping me to translate the popular scientific summary.

- Ali Fazli for the great collaboration on teaching. By the way, how is the life as a postdoc in USA? 
- Daniel Svärd for helping me to solve any 'unsolvable' computer-related problems.

- Ted Johansson for sharing his experience and for the help on job search.

- J. Jacob Wikner for his tough graduate courses and interesting posts. I collected only a few credits from his courses, but I laughed a lot reading his posts.

- Anna Folkesson and Maria Hamnér, who have been invaluable in their efforts to simplify all the administrative details.

- all former and present members of the Division of Electronic Devices, especially Amin Ojani, Behzad Mesgarzadeh, Duong Quoc Tai, Fahad Qazi, Jerzy Dabrowski, Kairang Chen, Omid Najari, and Shakeel Ahmad for being nice colleagues and creating a great research environment.

- all my friends for the time we spent together.

- Yuexian. Always back me up and have done so much for me!

- last but not least: Min for sharing the pleasures and sorrows of life with me; my mother Yuehua and grand-parents Shanfu and Meigui for giving me unconditional love and support.

Finally, profound thanks to those not listed here for bringing pleasant moments in my life.

Dai Zhang

Linköping, June 2014 


\section{Contents}

1 Introduction 1

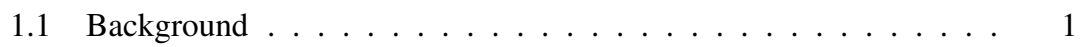

1.2 Review of Power-Efficient ADC Architectures . . . . . . . . . . 2

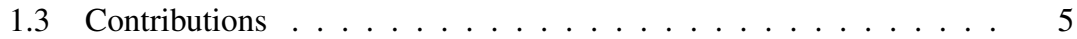

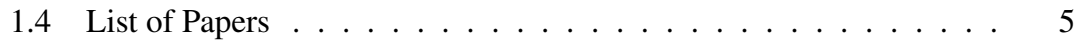

1.5 Thesis Organization . . . . . . . . . . . . . . . . 6

2 SAR ADC Design Considerations 7

2.1 Sampling Circuit $\ldots \ldots \ldots \ldots \ldots \ldots \ldots \ldots \ldots$

2.1.1 Thermal Noise . . . . . . . . . . . . . . . . . . 7

2.1 .2 Aperture Error . . . . . . . . . . . . . . . 8

2.1.3 Switch-Induced Error . . . . . . . . . . . . . . . . . . 9 9

2.1 .4 Track Bandwidth . . . . . . . . . . . . . . . . . . . 10

2.1 .5 Voltage Droop . . . . . . . . . . . . . . 11

2.2 Capacitive DAC . . . . . . . . . . . . . . . . 11

2.2.1 Single Binary-Weighted Capacitive Array . . . . . . . . . 11

2.2.2 Split Binary-Weighted Capacitive Array . . . . . . . . . . 13

2.3 Comparator . . . . . . . . . . . . . . . . . . 17

2.3.1 Preamplification Stage . . . . . . . . . . . . . . . . 17

2.3.2 Dynamic Latch . . . . . . . . . . . . . . 21

3 SAR ADC Power Analysis 27

3.1 Capacitive DAC . . . . . . . . . . . . . . . . . 28

3.2 Single-Pole Amplifier . . . . . . . . . . . . . . . . . . . . . . . . . . 29

3.3 Latch Comparator . . . . . . . . . . . . . . . . . . . 30

3.4 SAR Control Logic . . . . . . . . . . . . . . . . . . 32

3.5 Power Consumption Bounds of an Entire ADC . . . . . . . . 34

4 Design of 10-bit 1-kS/s SAR ADCs 37

4.1 A 53-nW 9.1-ENOB SAR ADC in 0.13 $\mu$ m CMOS Process . . . . . 38

4.1 .1 Introduction . . . . . . . . . . . . . . . 38 
4.1 .2 ADC Architecture . . . . . . . . . . . . . . 39

4.1 .3 Capacitive DAC . . . . . . . . . . . . . . 41

4.1 .4 Switch Design . . . . . . . . . . . . . . . . . 42

4.1 .5 Dynamic Latch Comparator . . . . . . . . . . . . . . . 44

4.1 .6 SAR Control Logic . . . . . . . . . . . . . . . . . . . . . . . . . . . . . . . . . . . 45

4.1.7 Measurement Results . . . . . . . . . . . . . . . . 46

4.2 A 3-nW 9.1-ENOB SAR ADC in 65nm CMOS Process . . . . . . . 50

4.2.1 Comparison in Leakage between $0.13 \mu \mathrm{m}$ and $65 \mathrm{~nm}$ CMOS Processes ................... 50

4.2 .2 ADC Architecture . . . . . . . . . . . . . . . 51

4.2 .3 Capacitive DAC . . . . . . . . . . . . . . . 53

4.2 .4 Switch Design . . . . . . . . . . . . . . . . . . 54

4.2.5 Dynamic Latch Comparator . . . . . . . . . . . . . . 56

4.2 .6 SAR Control Logic . . . . . . . . . . . . . . . . . . 57

4.2 .7 Chip Implementation . . . . . . . . . . . . . . . . . . . . . . . . . . . . . . . . . 58

4.2 .8 Measurement Results . . . . . . . . . . . . . . . . . 59

5 Digital Calibration of Capacitive DAC 63

5.1 Introduction . . . . . . . . . . . . . . . . 63

5.2 Capacitor Property $\ldots \ldots \ldots 65$

5.2.1 Capacitor Voltage Dependence . . . . . . . . . . . . . 65

5.2.2 Capacitor Temperature Dependence . . . . . . . . . . 65

5.3 Analysis of Non-Binary-Weighted DAC . . . . . . . . . . . . . . 66

5.3.1 Preliminaries ..................... 66

5.3.2 Accounting for Capacitor Variation . . . . . . . . . . . 67

5.3.3 Secondary Bit to Tackle One-Side Redundancy . . . . . . . 68

5.4 Bottom-Up Weight Calibration . . . . . . . . . . . . . . . . 71

5.4.1 Weight Conversion . . . . . . . . . . . . 72

5.4.2 Bottom-Up Weight Calibration . . . . . . . . . . . . 73

5.4 .3 Simulation Results . . . . . . . . . . . . . . . . . . 74

6 A 14-Bit Redundant SAR ADC in 65nm CMOS Process 79

6.1 Introduction . . . . . . . . . . . . . . . . . . . . . . . . . . . . 79

6.2 ADC Architecture . . . . . . . . . . . . . . . . . . . . . . 80

6.3 Circuit Implementation . . . . . . . . . . . . . . . . . . . . . . . . 83

6.3 .1 Capacitive DAC . . . . . . . . . . . . . 83

6.3 .2 Comparator . . . . . . . . . . . . . 85

6.3 .3 Biasing Circuit . . . . . . . . . . . . . . . . . . . 88

6.3 .4 Sampling Switch . . . . . . . . . . . . . . . . . . . . 89

6.3.5 Digital Control Logic . . . . . . . . . . . . . . . . . . . . . . . . 91

6.4 Measurement Results . . . . . . . . . . . . . . . . . 92 
7 Conclusions and Future Directions $\quad 99$

7.1 Conclusions . . . . . . . . . . . . . . . . . . . 99

7.2 Future Directions . . . . . . . . . . . . . . . . . 100

A Calculation of the Bridge Capacitor (Eq. (6.1)) 103

$\begin{array}{ll}\text { B Paper Collections } & 105\end{array}$

$\begin{array}{ll}\text { References } & 133\end{array}$ 


\section{List of Figures}

1.1 A simplified block diagram of a biomedical system. . . . . . . . . . 2

1.2 A simplified block diagram of a biopotential acquisition system including an AFE and an ADC. . . . . . . . . . . . . . . 2

1.3 Voltage and frequency ranges of four classes of bioelectric signals, where EOG, EEG, ECG, and EMG refer to the electrooculogram, the electroencephalogram, the electrocardiogram, and the electromyogram, respectively. . . . . . . . . . . . . . 2

1.4 Published ADCs in ISSCC [7]: (a) Power versus Nyquist sampling rate; (b) Power versus SNDR. . . . . . . . . . . . . . . . . . . . . . 3

1.5 A basic first-order $\Sigma \Delta$ ADC . . . . . . . . . . . . . . . 4

1.6 A basic SAR ADC . . . . . . . . . . . . . . . . . 4

2.1 Sampling circuit:(a) basic circuit (b) switch on-resistance versus input

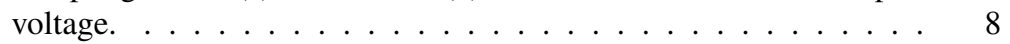

2.2 Aperture error. . . . . . . . . . . . . . . . 8

2.3 Sources of switch-induced error of sampling circuit. . . . . . . . . . 9

2.4 Sampling circuit acting as a first-order low-pass RC network and its transfer curve. . . . . . . . . . . . . . . . . . . . 10

2.5 A single binary-weighted capacitive DAC . . . . . . . . . . . . . 12

2.6 A split binary-weighted capacitive DAC . . . . . . . . . . . . . 13

2.7 A modified split binary-weighted capacitive DAC to avoid fractional value of bridge capacitor. . . . . . . . . . . . . . . . . 13

2.8 A simplified DAC circuit with the whole sub-DAC connected to ground. 14

2.9 A simplified DAC circuit with the whole main-DAC connected to ground. . . . . . . . . . . . . . . 15

2.10 Unit capacitance and total array capacitance versus main-DAC resolution. . . . . . . . . . . . . . . . . . 16

2.11 A single-pole preamplifier. . . . . . . . . . . . . . . . 17

2.12 Small signal-model of the single-pole preamplifier. . . . . . . . . . 18

2.13 Normalized response time of multi-stage amplifier as a function of $N .19$

2.14 A dynamic latch comparator [27]. . . . . . . . . . . . . . 21 
2.15 Simplified latch circuit and its small-signal model at regeneration mode. 22

2.16 Comparison of transient response among SPA, MSA, and latch. . . . 23

3.1 Charge-redistribution SAR ADC [35]. . . . . . . . . . . . . 27

3.2 Typical signal transient behavior including the differential outputs and the supply current. Note that there is no static supply current. . . 30

3.3 Comparison of energy consumption between SPA and latch. . . . . 32

3.4 A typical design of SAR control logic . . . . . . . . . . . . 33

3.5 A DFF with pass-gate style. . . . . . . . . . . . . . . . 33

3.6 Analyzed energy bounds of an entire ADC with its individual blocks: (a) DAC is noise-limited (b) DAC is mismatch-limited. . . . . . . . 35

3.7 $V_{\text {eff }}$ versus $V_{G T}$ for minimum-sized NMOS transistor in 65-nm and 130-nm technologies. . . . . . . . . . . . 36

4.1 Simulated average power consumption versus switching frequency of an inverter with a fan-out of four in $0.13-\mu \mathrm{m}$ CMOS process. . . . . 38

4.2 Architecture of the SAR ADC . . . . . . . . . . . . . . . 39

4.3 The sampling phase of capacitive DAC with MSB preset. . . . . . . 40

4.4 Waveform of the DAC switching procedure. . . . . . . . . . . . 41

4.5 Layout of the capacitor array which follows a partial common-centroid configuration. The capacitors are indicated according to Fig. 4.2. . . 41

4.6 Top-plate sampling switch. . . . . . . . . . . . . . . . . . 42

4.7 Simulated leakage current of the sampling switch: (a) test-bench (b) leakage current versus input voltage. . . . . . . . . . . . . . . 43

4.8 Dynamic latch comparator [49]. . . . . . . . . . . . . . . . . . 44

4.9 SAR control logic. . . . . . . . . . . . . . . . . . 45

4.10 Time sequence of the synchronous SAR control logic. . . . . . . . . 46

4.11 A conventional level shifter. . . . . . . . . . . . . . . . 46

4.12 Die photograph of the ADC in $0.13-\mu$ m CMOS technology. . . . . . 47

4.13 Measured DNL and INL errors. . . . . . . . . . . . . . . . . . 47

4.14 Dynamic performance: (a) Measured 8,192-point FFT spectrum at $1 \mathrm{kS} / \mathrm{s}$ (b) ENOB versus input frequency. . . . . . . . . . . . . . . 48

4.15 The ADC power breakdown in dual and single supply modes, where the percentage of digital leakage power is indicated by dark color.

4.16 Simulated transistor sub-threshold leakage current versus channel length with minimum width at $1-\mathrm{V}$ supply, typical corner, and $27^{\circ} \mathrm{C}$ : (a) in standard $0.13 \mu \mathrm{m}$ CMOS process; (b) in low-power $65 \mathrm{~nm}$ CMOS process. . . . . . . . . . . . . . 50

4.17 SAR ADC architecture . . . . . . . . . . . . . . . . . 51

4.18 Time sequence of SAR ADC . . . . . . . . . . . . . . 52

4.19 DAC arrays during (a) reset phase (b) sampling phase. . . . . . . . 52

4.20 Layout floor plan of the capacitor array. The capacitors, not indicated in the figure are dummies. . . . . . . . . . . . . . . . 54 
4.21 The parasitic capacitance of the bridge capacitor: 1) connected to the main-DAC (Case 1); 2) connected to the sub-DAC (Case 2). . . . . 54

4.23 Voltage boosting circuit with bypass function. . . . . . . . . . . 55

4.22 (a) Top-plate switches. (b) Bottom-plate switches. . . . . . . . . . . 56

4.24 Dynamic latch comparator with high- and standard- $\mathrm{V}_{\mathrm{T}}$ transistors. . 57

4.25 SAR digital control logic. . . . . . . . . . . . . . . . . . 58

4.26 Die photo and layout view of the ADC in $65 \mathrm{~nm}$ CMOS process. . . 59

4.27 Measured DNL and INL errors of 1-kS/s 0.7-V ADC. . . . . . . . . 60

4.28 Measured 8,192-point FFT spectrums of 1-kS/s 0.7-V ADC: (a) near DC (b) near Nyquist. . . . . . . . . . . . . . . . . . . . 60

4.29 Predicted mismatch-limited SAR ADC energy bounds (solid line) together with Nyquist SAR ADC survey data $(\Delta)$ and the two implemented ADCs $(0) \ldots \ldots \ldots \ldots \ldots \ldots \ldots$

5.1 Decision tree of: (a) a binary-weighted converter; (b) a non-binaryweighted converter. . . . . . . . . . . . . . . . 64

5.2 Tradeoffs among $\sigma_{u} / C_{u}, r$, and $S$ for 15-bit accuracy. . . . . . 68

5.3 Decision path (indicated by black solid line) after a wrong decision at MSB approximation for two normalized input voltages: (a) $V_{I N}=0.46$ (b) $V_{I N}=0.4 \ldots \ldots \ldots \ldots$

5.4 A SAR ADC incorporated weight conversion (parts indicated by grey color are the additional blocks). . . . . . . . . . . . . .

5.5 Test logic to generate the calibration signals $\left(T_{L} \sim T_{S}\right)$ and the corresponding time sequence. . . . . . . . . . . . 71

5.6 DAC switching status during conversion of MSB weight $\left(W_{S}\right)$ : (a) Sampling; (b) 1st-bit approximation; (c) 2nd-bit approximation. . . 72

5.7 Weight conversion with the existence of offset. . . . . . . . . . . 74

5.8 Block diagram of the digital calibration logic. . . . . . . . . . . . . 74

5.9 Simulated SNDR versus numbers of calibrated higher weights for $\sigma_{u} / C_{u}=3 \%$ : (a) mean (b) standard deviation. . . . . . . . . . . .

5.10 Comparison between before and after calibration: (a) simulated SNDR (mean-3 $\sigma$ ) (b) improved SNDR after calibration. (The total number of calibrated weights are indicated.)

$5.11 \mathrm{DNL} / \mathrm{INL}$ plots of an ADC with $\sigma_{u} / C_{u}=1.5 \%$ : (a) before calibration (b) after calibration of 9 bits. . . . . . . . . . . 76

6.1 SAR ADC architecture. . . . . . . . . . . . . . . . . 80

6.2 The first three DAC switching phases of a 3-bit SAR ADC: (a) employing top-plate sampling; (b) employing bottom-plate sampling. . $\quad 82$

6.3 The DAC with calibration circuits. . . . . . . . . . . . . . . 83

6.4 Split DAC with its non-binary-weighted numbers rounded to the nearest integer . . . . . . . . . . . . . . . 
6.5 Cross view of multi-layer sandwich capacitor and its simplified schemica view. . . . . . . . . . . . . . . . . . . . 84

6.6 Guard ring of P-substrate around the capacitive array for noise isolation. 84

6.7 Part of the DAC layout showing the connections of the bridge capacitor. 85

6.8 Block diagram of the comparator. . . . . . . . . . . . . . . . . . 85

6.9 Schematic diagram of the preamplifier. . . . . . . . . . . . 86

6.10 Block diagram of the bias control circuit. . . . . . . . . . . . . 87

6.11 Time sequence of the bias control circuit. . . . . . . . . . . . . . . . . 88

6.12 Schematic view of the biasing circuit. . . . . . . . . . . . . . 89

6.13 Simplified gate-source bootstrapping circuit. . . . . . . . . . . . . . . 89

6.14 Two phases of gate-source bootstrapping circuit. . . . . . . . . . . . 90

6.15 Schematic view of the bootstrapping circuit [73] . . . . . . . . . . 90

6.16 Block diagram of SAR control logic. . . . . . . . . . . . . . . . . 91

6.17 Gates used in the combinational switching logic. . . . . . . . . . . 92

6.18 Chip photo of the ADC in 65nm CMOS technology. . . . . . . . . . 92

6.19 Measured DNL and INL error according to 14-bit resolution at $1 \mathrm{~V}$ : (a) before calibration (b) after calibration. . . . . . . . . . . . 93

6.20 Measured 16384-point FFT spectrums of 10-kS/s 1-V ADC: (a) near DC (b) near Nyquist. . . . . . . . . . . . . . . . . . . . . 93

6.21 SFDR, SNR, and SNDR versus input frequency at 1 V. . . . . . . . 94

6.22 Measured 16384-point FFT spectrums of 10-kS/s 0.8-V ADC: (a) near DC (b) near Nyquist. . . . . . . . . . . . . . . . . . . . . . . 94

6.23 SFDR, SNR, and SNDR versus input frequency at 0.8 V. . . . . . . . 94

6.24 Measured DNL and INL error according to 14-bit resolution at $0.8 \mathrm{~V} .95$

6.25Walden FOM and thermal FOM versus SNDR@ Nyquist of Nyquist SAR ADCs presented at ISSCC from data in [7]. . . . . . . . . . . 96

6.26 Predicted mismatch-limited SAR ADC energy bounds (solid line) together with Nyquist SAR ADC survey data $(\Delta)$ and the implemented ADCs $(0) \ldots \ldots \ldots \ldots$. . . . . . . . . . . . . 97

7.1 ECG signal. . . . . . . . . . . . . . . . . . . . 101

7.2 An additional capacitor and an integrator are added to the SAR architecture to perform 1st-order noise-shaping. . . . . . . . . . . . . . 101

7.3 Linear model transfer functions of a 1st-order noise-shaping SAR ADC.101

A.1 Split capacitive DAC with one bridge capacitor. . . . . . . . . . . . 103 


\section{List of Tables}

3.1 Parameter Values Used in Demonstration . . . . . . . . . . . 35

4.1 ADC Measurement Summary . . . . . . . . . . . . . . . . . . . . 49

4.2 ADC Performance Under Different Supply Settings . . . . . . . . . 50

4.3 ADC Dynamic Performance With Respect to Bridge Capacitor Con-

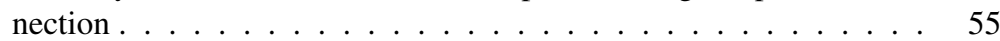

4.4 Simulated Power Consumption . . . . . . . . . . . . . . . . . . . 58

4.5 ADC Measurement Summary . . . . . . . . . . . . . . . . 61

4.6 Measured ADC Performance Under Different Supply Voltages . . . 61

4.7 ADC Comparison . . . . . . . . . . . . . . . . 61

$5.1 \quad$ Required Minimum $S$ in Terms of $r$ for $N=15 \ldots \ldots 68$

5.2 Maximum $r$ Can be Used in Terms of $j \ldots \ldots$. . . . . . . 68

5.3 Distance Between Two Bits in Terms of Radix . . . . . . . . . . . . 70

5.4 Comparison With Other Digital Calibration Techniques . . . . . . 77

6.1 Comparison Among Three Split Topologies . . . . . . . . . . . . . 81

6.2 Comparison of Simulated Amplifier Performance under Two Bias Conditions . . . . . . . . . . . . . . . . . 87

6.3 Measured ADC Power Consumption . . . . . . . . . . . . . . . . . . . . 95

6.4 ADC Measurement Summary . . . . . . . . . . . . . . . . . . . . . . 96

6.5 ADC Comparison . . . . . . . . . . . . . . . . . . . . 97 


\section{List of Abbreviations}

ADC

AFE

CMOS

CMRR

DAC

DFF

DNL

DSP

ECG

EEG

EMG

ENOB

EOG

ERBW

FFT

FOM

IA

INL

ISSCC
Analog-to-Digital Converter

Analog Front End

Complementary Metal Oxide Semiconductor

Common-Mode Ripple Rejection

Digital-to-Analog Converter

D-type Flip Flop

Differential Nonlinearity

Digital Signal Processor

Electrocardiogram (measurements of heart function)

Electroencephalogram (measurements of brain function)

Electromyogram (measurements of muscle function)

Effective Number of Bit

Electrooculogram (measurements of eye motion)

Effective Resolution Bandwidth

Fast Fourier Transform

Figure of Merit

Instrumentation Amplifier

Integral Nonlinearity

International Solid-State Circuits Conference 


\begin{tabular}{ll} 
JLCC & J-Leaded Chip Carrier \\
KCL & Kirchhoff's Current Law \\
LPF & Low-Pass Filter \\
LSB & Least Significant Bit \\
MIM & Metal Insulator Metal \\
MSB & Most Significant Bit \\
PGA & Programmable Gain Amplifier \\
SAR & Successive Approximation Register \\
SFDR & Spurious-Free Dynamic Range \\
SMR & Signal-to-Metastability-Error Ratio \\
SNDR & Signal-to-Noise-and-Distortion Ratio \\
SNR & Signal-to-Noise Ratio \\
SPA & Single-Pole Amplifier \\
THD & Total Harmonic Distortion \\
\hline
\end{tabular}




\section{Chapter 1}

\section{Introduction}

\subsection{Background}

Wearable and implantable biomedical systems [1-5] are advancing the development of healthcare. Such systems enable chronic disease management, medical diagnosis, and fitness tracking outside of a traditional clinical setting, thereby bringing a high quality-of-life to people with reduced costs. Fig. 1.1 shows a simplified block diagram of such systems. An analog front end (AFE) senses the bioelectric signals. The sensed signal is first digitized by an analog-to-digital converter (ADC) and then processed in a digital back end. If necessary, a radio is succeeded to transmit data to a central base-station node. Since these systems are commonly attached to or implanted into human bodies and powered by harvested energy or small batteries, ultra-low-power operation becomes paramount.

Figure 1.2 shows a simplified block diagram of a biopotential acquisition system which comprises an AFE and an ADC. The AFE is composed of an instrumentation amplifier (IA), a programmable gain amplifier (PGA), and an anti-aliasing low-pass filter. The bioelectric signals, shown in Fig. 1.3, have dynamic range between tens of microvolt to hundreds of millivolt, and they cover a frequency band with the highest frequency less than $10 \mathrm{kHz}$ [6]. To extract the low-level bioelectric signals, the IA filters the differential electrode offset, and defines a low noise performance and a high common-mode-ripple-rejection (CMRR) of the AFE [1]. The PGA is used to adjust the gain and bandwidth of the readout for different bioelectric signals. In certain cases, to meet with stringent power budgets, the PGA can also perform the operation of an anti-aliasing filter [1]. Finally, the bioelectric signals after sensing, filtering, and amplifying by the AFE are strong enough to be digitized in the succeeding ADC.

$\mathrm{ADC}$ is a key component as the interface between the analog world and the digital domain. Conversion of the low-frequency bioelectric signals does not require high speed, but ultra-low-power consumption. This combined with the required conversion 
accuracy makes the design of such ADCs a major challenge. This thesis examines the physical limitations and investigates the design methodologies and circuit techniques for low-speed and ultra-low-power ADCs.

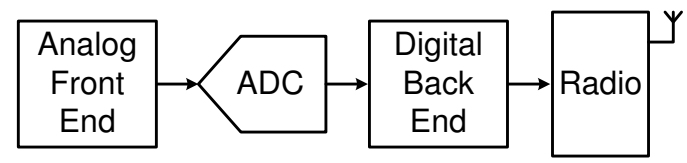

Figure 1.1: A simplified block diagram of a biomedical system.

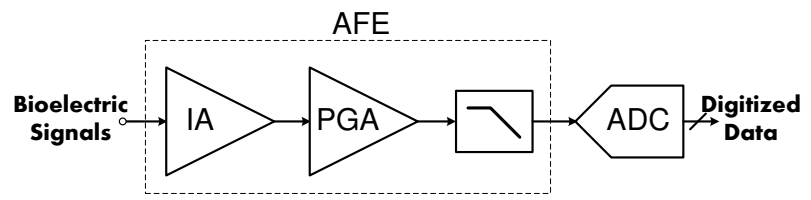

Figure 1.2: A simplified block diagram of a biopotential acquisition system including an AFE and an ADC.

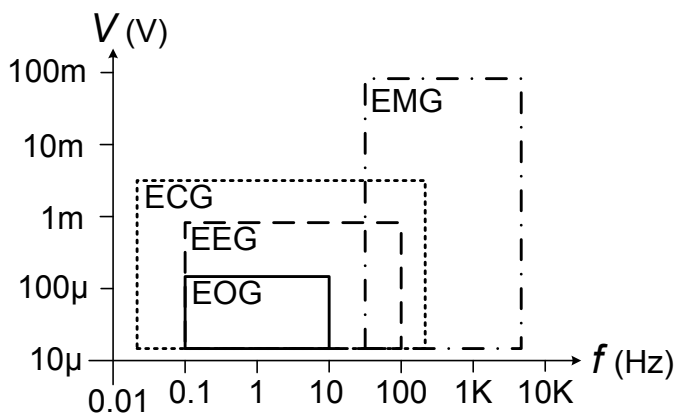

Figure 1.3: Voltage and frequency ranges of four classes of bioelectric signals, where EOG, EEG, ECG, and EMG refer to the electrooculogram, the electroencephalogram, the electrocardiogram, and the electromyogram, respectively.

\subsection{Review of Power-Efficient ADC Architectures}

Since ultra-low-power operation is critical, architecture selection is driven by an examination of the power consumption of prevalent ADCs. Fig. 1.4 plots the power consumption of published ADCs versus the sampling rate and the signal-to-noise-anddistortion ratio (SNDR), respectively. As shown in Fig. 1.4, successive approximation register (SAR) ADCs and sigma-delta $(\Sigma \Delta)$ ADCs are typically used for low-speed, 
medium-to-high resolution applications. Pipelined ADCs dominate at medium-speed and medium-resolution domain, and flash ADCs at high-speed and low-resolution domain. With respect to the desired speed and resolution, $\Sigma \Delta$ and SAR ADCs become primary candidates.

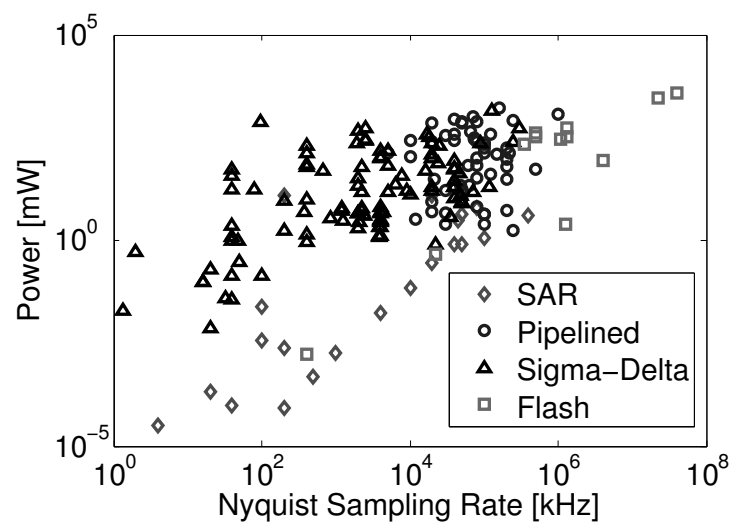

(a)

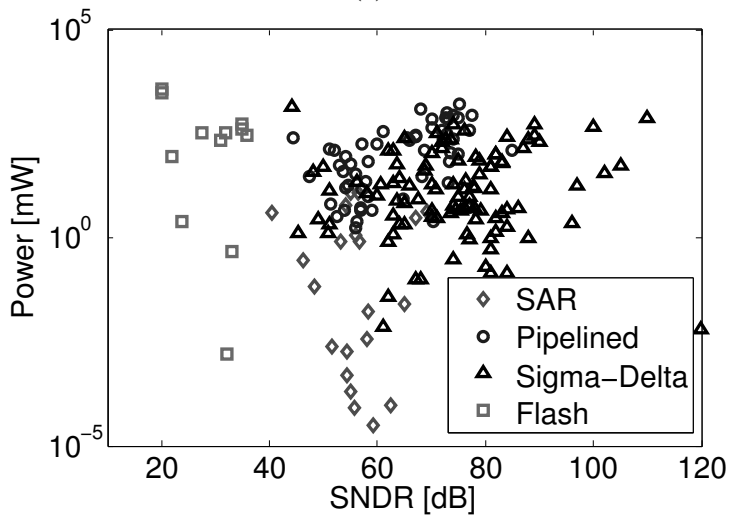

(b)

Figure 1.4: Published ADCs in ISSCC [7]: (a) Power versus Nyquist sampling rate; (b) Power versus SNDR.

Figure 1.5 shows the topology of a basic first-order $\Sigma \Delta$ ADC. An integrator and a comparator are in the forward path, and a 1-bit DAC is in the feedback path. The transfer function from $V_{I N}$ to $V_{O U T}$ follows that of a low-pass filter. While, the transfer function of the quantization noise follows that of a high-pass filter, thereby pushing the noise out of the signal bandwidth. The modulator is succeeded with a lowpass filter (LPF) which removes the out-of-band quantization noise and downsamples 
the signal. The oversampling feature of $\Sigma \Delta$ modulation eases the anti-aliasing requirements. In addition, the noise-shaping technique makes $\Sigma \Delta$ ADCs dominate in the high-resolution regime [8-10].

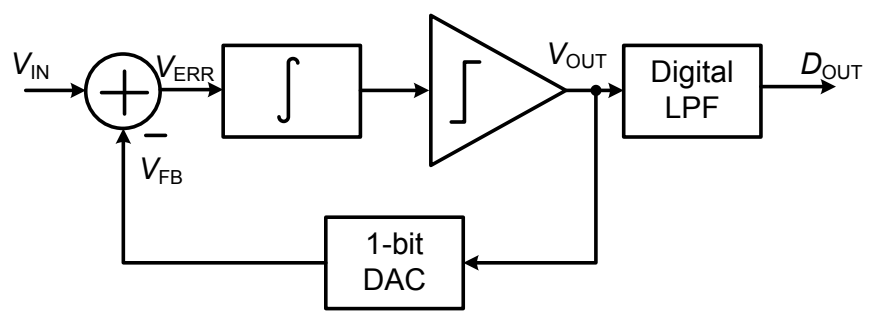

Figure 1.5: A basic first-order $\Sigma \Delta$ ADC.

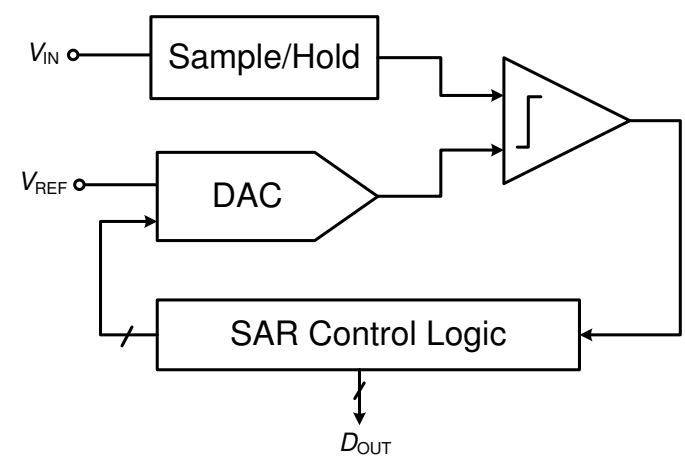

Figure 1.6: A basic SAR ADC.

Figure 1.6 shows the architecture of a basic SAR ADC. It consists of a sampleand-hold circuit, a digital-to-analog converter (DAC), a comparator, and a digital control logic. The SAR ADC works based on the binary-search algorithm. First, the input voltage is sampled. Then the conversion starts with an approximation of the most-significant-bit (MSB) and continues until the least-significant-bit (LSB) is decided. For an N-bit SAR ADC it usually takes at least $\mathrm{N}$ clock cycles to complete one conversion. Since there is only one comparator and no other active components in the converter, SAR ADCs are highly power-efficient [11-13]. Moreover, owing to its dynamic nature, SAR ADCs are also amenable to technology scaling [14].

In parallel to the research track of $\Sigma \Delta$ ADCs for medical applications [15], this thesis focuses on the SAR architecture. 


\subsection{Contributions}

The main contributions of this thesis are as follows:

- Analysis of the power consumption bounds for SAR ADCs.

- Design and implementation of a 53-nW 9.1-ENOB 1-kS/s SAR ADC in $0.13 \mu \mathrm{m}$ CMOS process.

- Design and implementation of a 3-nW 9.1-ENOB 1-kS/s SAR ADC in 65nm CMOS process.

- Development of a bottom-up weight calibration method to mitigate the DAC mismatch of high-resolution SAR ADCs. The method was verified on a redundant SAR ADC behavioral model.

- Design and implementation of a 1.98- $\mu \mathrm{W}$ 12.5-ENOB 10-kS/s redundant SAR $\mathrm{ADC}$ in $65 \mathrm{~nm}$ CMOS process.

\subsection{List of Papers}

This thesis is a result of the research performed at the Division of Electronic Devices, Department of Electrical Engineering, Linköping University between April 2009 and June 2014. Part of the contents of this thesis have been presented in the following publications:

- Dai Zhang, Ameya Bhide, and Atila Alvandpour, "A 53-nW 9.1-ENOB 1-kS/s SAR ADC in 0.13- $\mu \mathrm{m}$ CMOS for Medical Implant Devices", in IEEE Journal of Solid-State Circuits, vol.47, no.7, pp.1585-1593, July 2012. [Special issue on ESSCIRC2011]

(Contributions: Ameya designed and implemented the SAR digital control logic; I designed and implemented the capacitive DAC and the comparator, and also did the chip integration and measurement.)

- Dai Zhang and Atila Alvandpour, "Analysis and Calibration of Non-BinaryWeighted Capacitive DAC for High-Resolution SAR ADCs", accepted for publication in IEEE Transactions on Circuits and System - II: Express Briefs.

- Dai Zhang and Atila Alvandpour, "A 3-nW 9.1-ENOB SAR ADC at 0.7 V and $1 \mathrm{kS} / \mathrm{s}$ ", in proceedings of the European Solid-State Circuit Conference (ESSCIRC), pp.369-372, Bordeaux, France, September 2012. 
- Dai Zhang, Ameya Bhide, and Atila Alvandpour, "A 53-nW 9.12-ENOB $1-\mathrm{kS} / \mathrm{s}$ SAR ADC in 0.13- $\mu \mathrm{m}$ CMOS for Medical Implant Devices", in proceedings of the European Solid-State Circuit Conference (ESSCIRC), pp.467-470, Helsinki, Finland, September 2011.

- Dai Zhang, Christer Svensson, and Atila Alvandpour, "Power consumption bounds for SAR ADCs", in proceedings of the European Conference on Circuit Theory and Design (ECCTD), pp.556-559, Linköping, Sweden, August 2011.

- Dai Zhang, Ameya Bhide, and Atila Alvandpour, "Design of CMOS sampling switch for ultra-low power ADCs in biomedical applications", in proceedings of the Norchip Conference, pp.1-4, Tempera, Finland, November 2010.

- Dai Zhang and Atila Alvandpour, "A 14b 10kS/s redundant SAR ADC with 77dB SNDR up to Nyquist in 65nm CMOS", manuscript to be submitted.

For ease of reference, the published papers are attached in Appendix B (except for the manuscript and the ESSCIRC paper which overlaps with the JSSC paper).

\subsection{Thesis Organization}

The rest of this thesis is organized as follows. Chapter 2 discusses the error sources and design considerations of SAR ADC blocks. Chapter 3 performs the analysis of power consumption bounds for SAR ADCs. Chapter 4 presents the design and implementation of two 1kS/s SAR ADCs: a 53-nW 9.1-ENOB SAR ADC in $0.13 \mu \mathrm{m}$ CMOS and a 3-nW 9.1-ENOB SAR ADC in 65nm CMOS. To mitigate the capacitor mismatch for high-resolution, Chapter 5 develops a bottom-up weight calibration method which is applied to a redundant SAR ADC. Chapter 6 presents the design and implementation of a 10kS/s redundant SAR ADC achieving 77dB SNDR with $1.98 \mu \mathrm{W}$ at $0.8 \mathrm{~V}$ in $65 \mathrm{~nm}$ CMOS process. Finally, Chapter 7 concludes the thesis and sketches the future directions. 


\section{Chapter 2}

\section{SAR ADC Design Considerations}

This chapter discusses the error sources and the design considerations of SAR ADC blocks including the sampling circuit, the capacitive DAC, and the comparator.

\subsection{Sampling Circuit}

A basic sampling circuit consists of a switch and a capacitor, shown in Fig. 2.1(a). When the switch is on, the input voltage is connected to the top-plate of the sampling capacitor. When the switch is off, the top-plate node of the capacitor is isolated, and the capacitor holds the sampled voltage. Fig. 2.1(b) shows the on-resistance versus the input voltage for three types of switches: PMOS, NMOS, and CMOS devices. Among the three devices, the CMOS device allows full-range input sampling and has the lowest on-resistance. The general design considerations of the sampling circuit are: thermal noise, aperture error, switch-induced error, track bandwidth, and voltage droop.

\subsubsection{Thermal Noise}

Thermal noise, introduced by the on-resistance of the switch, is given by $k T / C_{S}$, where $k$ is the Boltzmann constant, $T$ is the absolute temperature, and $C_{S}$ is the sampling capacitor. As is known, the quantization noise sets a fundamental limit on the SNR of the ADC. If we consider an $N$-bit ADC with a full-scale voltage of $V_{F S}$, the quantization noise is given by

$$
v_{q n}^{2}=\frac{V_{F S}^{2}}{12 \cdot 2^{2 N}} .
$$




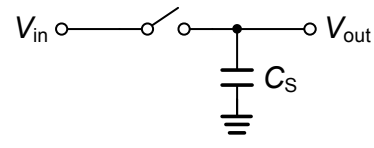

(a)

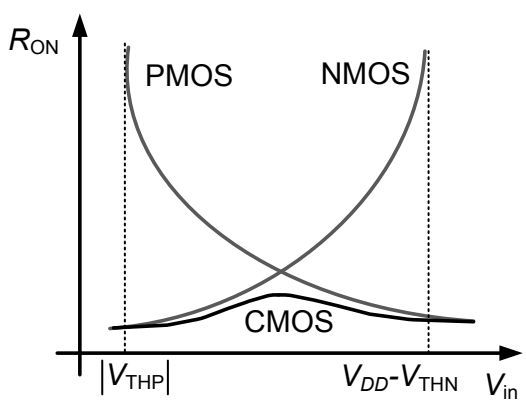

(b)

Figure 2.1: Sampling circuit:(a) basic circuit (b) switch on-resistance versus input voltage.

In a Nyquist $\mathrm{ADC}$, the sampling capacitor is typically chosen large enough so that the thermal noise is less than the converter's quantization noise, which gives a minimum value of $C_{S}$

$$
C_{S}=12 k T \frac{2^{2 N}}{V_{F S}^{2}} .
$$

\subsubsection{Aperture Error}

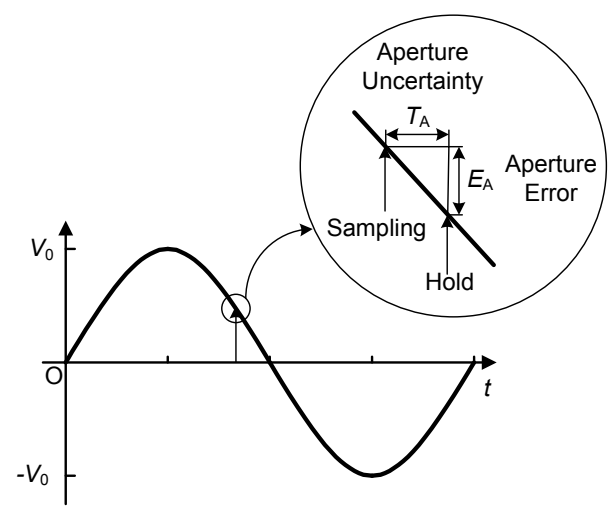

Figure 2.2: Aperture error.

Aperture error is caused by the uncertainties in the time domain from sample mode to hold mode, as shown in Fig. 2.2. This variation is mainly due to the noise on 
the sampling clock. The aperture error voltage, denoted as $E_{A}$, depends on the slew rate of the input signal and the aperture uncertainty, denoted as $T_{A}$. For a sinusoidal signal, the maximum slew rate occurs at the zero crossing point and is given by

$$
\left.\frac{d V}{d t}\right|_{\max }=2 \pi f_{I N} V_{0},
$$

where $f_{I N}$ is the input frequency and $V_{0}$ is the input amplitude. To ensure the aperture error to be less than $1 / 2 \mathrm{LSB}$ at the point of maximum slew rate, it requires

$$
2 \pi f_{I N} V_{0} T_{A}<\frac{1}{2} L S B
$$

Following Eq. (2.4), the maximum input frequency is given by

$$
f_{I N @ M A X}=\frac{1}{\pi \times 2^{N+1} \times T_{A}} .
$$

\subsubsection{Switch-Induced Error}

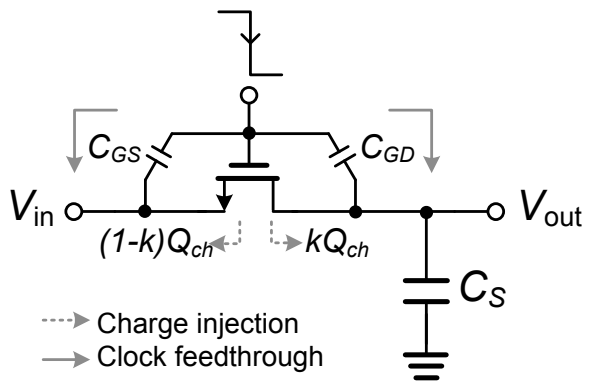

Figure 2.3: Sources of switch-induced error of sampling circuit.

Charge injection and clock feedthrough (shown in Fig. 2.3), collectively known as the switch-induced error, happen at the moment of turning off the switch. Charge injection introduces error to the sampled voltage by depositing part of the charge from the conduction channel of the transistor onto the sampling capacitor. Clock feedthrough affects the sampled voltage by capacitance coupling during the transition of the sampling clock. The switch-induced error voltage for both NMOS and PMOS can be approximated as [16]

$$
\Delta V_{e, N}=-\frac{k W_{N} L_{N} C_{O X}\left(V_{D D}-V_{T H N}-V_{I N}\right)}{C_{S}}-\frac{C_{G D, N}}{C_{S}+C_{G D, N}} V_{D D},
$$




$$
\Delta V_{e, P}=\frac{k W_{P} L_{P} C_{O X}\left(V_{I N}-\left|V_{T H P}\right|\right)}{C_{S}}+\frac{C_{G D, P}}{C_{S}+C_{G D, P}} V_{D D},
$$

where $k$ is the fraction of charge injected on the output node, $C_{O X}$ is the gate-oxide capacitor, $V_{T H N}$ and $V_{T H P}$ are the threshold voltages, and $C_{G D, N}$ and $C_{G D, P}$ are the gate-drain overlap capacitance of NMOS and PMOS, respectively. In Eq. (2.6) and Eq. (2.7), the first part of the right-half side represents the charge injection error, which varies with the input signal in a linear fashion if the body effect is neglected. The second part represents the clock feedthrough error, which is input-independent and can be taken as an offset error.

\subsubsection{Track Bandwidth}

The sampling circuit acts as a first-order low-pass RC network, shown in Fig. 2.4. Its track bandwidth is expressed as

$$
f_{3 d B}=\frac{1}{2 \pi \tau},
$$

where $\tau$ is the time constant. The time for the sampled voltage to settle with an error less than $1 / 2 \mathrm{LSB}$ can be derived from

$$
e^{-\frac{t}{\tau}}<\frac{1}{2^{N+1}}
$$
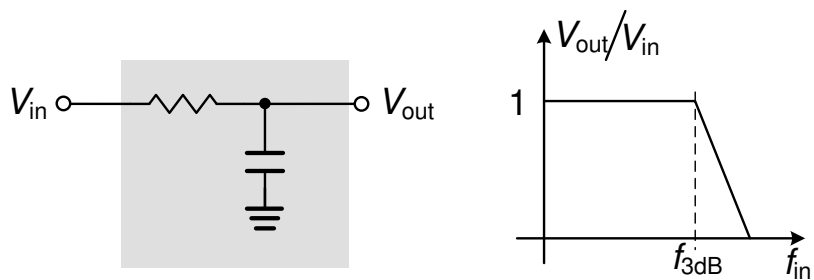

Figure 2.4: Sampling circuit acting as a first-order low-pass RC network and its transfer curve.

Substituting the time constant in Eq. (2.9) with Eq. (2.8), and assuming half sampling clock is used for settling, the track bandwidth should satisfy

$$
f_{3 d B}>\frac{\ln 2 \times(N+1)}{\pi} f_{S},
$$

where $f_{S}$ is the sampling frequency. 
Apart from the settling, the maximum input frequency will also set a requirement on the $3 \mathrm{~dB}$ bandwidth. The transfer function of the sampling circuit can be written as

$$
\frac{V_{\text {out }}}{V_{\text {in }}}(s)=\frac{1}{1+\frac{s}{\omega_{3 d B}}} .
$$

To keep the error of the sampled voltage less than $1 / 2 \mathrm{LSB}$, it requires

$$
\sqrt{\frac{1}{1+\left(\frac{f_{i n}}{f_{3 d B}}\right)^{2}}}>1-\frac{1}{2^{N+1}} .
$$

With further mathematical manipulation, the minimum $f_{3 d B}$ determined by the input frequency can be written as

$$
f_{3 d B}>2^{\frac{N}{2}} f_{i n}
$$

\subsubsection{Voltage Droop}

Voltage droop introduced by the leakage current of the switch becomes a critical error source when the sampling rate goes low. The subthreshold leakage current of the transistor is the dominant contributor and is expressed as [17]

$$
I_{D S}=\mu_{0} C_{O X} \frac{W}{L}(m-1) V_{T}^{2} \times e^{\frac{V_{G S}-V_{T H}}{m V_{T}}} \times\left(1-e^{-\frac{V_{D S}}{V_{T}}}\right),
$$

where $m$ is the subthreshold swing coefficient and $V_{T}$ is the thermal voltage. The subthreshold leakage current shows nonlinear dependence on the input-output voltage difference across the switch $\left(V_{D S}\right)$ and will introduce harmonic distortions.

\subsection{Capacitive DAC}

Capacitive DAC is commonly used to generate wighted reference voltages. Compared to a resistive DAC, the capacitor array is better for matching and more power-efficient. In this section, two commonly-employed capacitive DAC architectures will be described: single binary-weighted array and split binary-weighted array.

\subsubsection{Single Binary-Weighted Capacitive Array}

Figure 2.5 shows a single binary-weighted capacitive DAC. During the reset mode, all the bottom-plate nodes are reset to ground and the top-plate node is connected to a reset voltage, allowing the capacitors to discharge. When comes to the conversion 


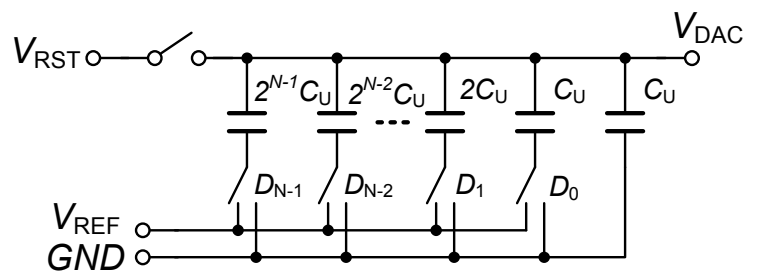

Figure 2.5: A single binary-weighted capacitive DAC.

mode, the digital codes determine the switch status, generating a weighted reference voltage.

The unit capacitor, denoted as $C_{u}$, should be kept as small as possible for power saving. In practice, it is usually determined by the thermal noise and the capacitor mismatch. The effect of thermal noise has been discussed in Sec. 2.1.1. Here, we will look at the mismatch.

Generally, the unit capacitor is modeled with a nominal value of $C_{u}$ and a standard deviation of $\sigma_{u}$ [18]. For a binary-weighted capacitor array, the worst-case standard deviation of differential nonlinearity (DNL) and integral nonlinearity (INL) occur at the MSB code transition due to the maximum accumulation of the capacitor mismatch, which can be expressed in terms of LSB as [19]

$$
\begin{gathered}
\sigma_{D N L, M A X}=\sqrt{2^{N}-1} \frac{\sigma_{u}}{C_{u}} L S B . \\
\sigma_{I N L, M A X}=\sqrt{2^{N-2}} \frac{\sigma_{u}}{C_{u}} L S B .
\end{gathered}
$$

Comparing Eq. (2.15) with Eq. (2.16), the derived worst-case standard deviation of DNL is larger than that of INL. Therefore, Eq. (2.15) is chosen to be a reference in the following analysis. For a typical metal-insulator-metal (MIM) capacitor, it has

$$
\begin{array}{r}
\sigma\left(\frac{\Delta C}{C}\right)=\frac{K_{\sigma}}{\sqrt{A}}, \\
C=K_{C} \cdot A,
\end{array}
$$

where $\sigma(\Delta C / C)$ is the standard deviation of capacitor mismatch, $K_{\sigma}$ is the matching coefficient, $A$ is the capacitor area, and $K_{C}$ is the capacitor density parameter. The standard deviation of a single capacitor to the nominal value is by factor $\sqrt{2}$ smaller than that of the difference between two capacitors, which can be expressed as

$$
\frac{\sigma_{u}}{C_{u}}=\frac{\sigma(\Delta C / C)}{\sqrt{2}} .
$$


For high yield, it is necessary to maintain $3 \sigma_{D N L, M A X}<1 / 2 L S B$. Combining Eq. (2.15), and Eq. (2.17) - Eq. (2.19), we obtain the lower bounds of the mismatchlimited unit capacitor

$$
C_{u}=18 \cdot\left(2^{N}-1\right) \cdot K_{\sigma}^{2} \cdot K_{C}
$$

So far, the discussion is for the single-ended architecture. For the differential configuration, the unit capacitance can be reduced by half while still satisfying the mismatch requirement. This is because the differential mode doubles the signal range but only increases $\sqrt{2}$ times of the error voltage introduced by the mismatch.

\subsubsection{Split Binary-Weighted Capacitive Array}

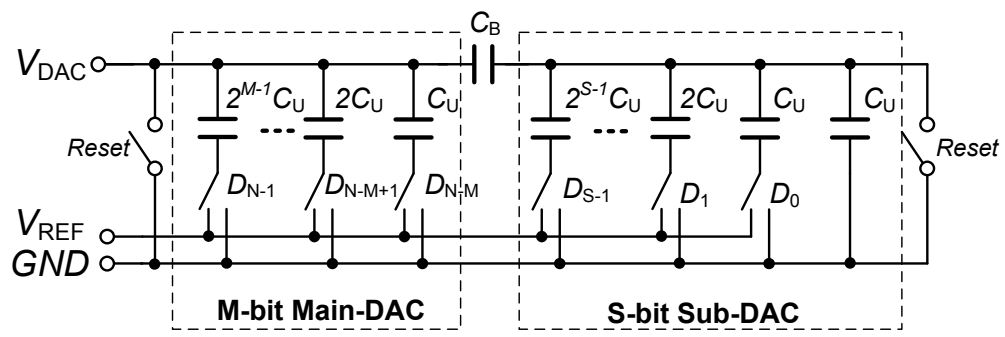

Figure 2.6: A split binary-weighted capacitive DAC.

A split binary-weighted capacitive DAC, shown in Fig. 2.6, is commonly used to reduce the total number of unit capacitors. It consists of an $M$-bit main-DAC and an $S$-bit sub-DAC, where $M+S=N$. Via a bridge capacitor, denoted as $C_{B}$ in Fig. 2.6, the sub-DAC interpolates between transition voltages generated by the main-DAC.

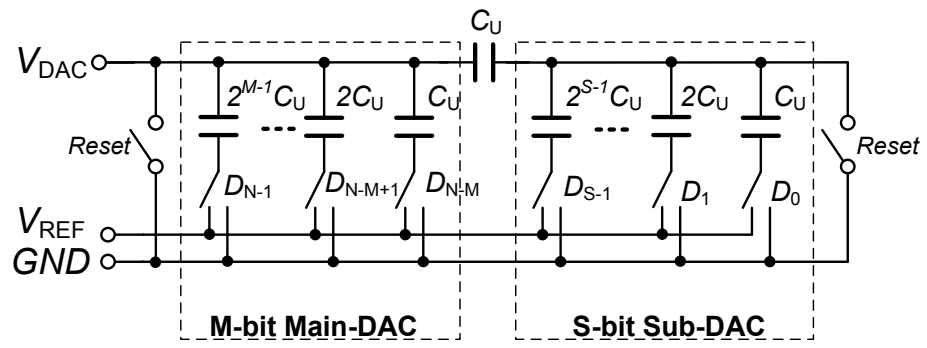

Figure 2.7: A modified split binary-weighted capacitive DAC to avoid fractional value of bridge capacitor. 
Assuming $M$ and $S$ are both set to 5, the bridge capacitor is calculated to be $32 / 31 C_{U}$. The fractional value of $C_{B}$ introduces layout difficulties and additional mismatch. Hence, to avoid the fractional value a modified split-DAC is employed, shown in Fig. 2.7, where the dummy capacitor of the sub-DAC is removed and the bridge capacitor is equal to the unit capacitor [20]. This modification will introduce gain error to the conversion, which will be discussed in the following section.

\subsubsection{Gain Error}

First, we consider the case shown in Fig. 2.8, where the entire sub-DAC is connected to ground and several capacitors in the main-DAC is connected to $V_{R E F}$. The voltage at $V_{M}$ is

$$
\begin{aligned}
V_{M} & =\frac{C_{V R E F}^{M}}{\left(2^{M}-1\right) C_{U}+\left(1-2^{-S}\right) C_{U}} V_{R E F} \\
& =\frac{C_{V R E F}^{M}}{2^{M}\left(1-2^{-N}\right) C_{U}} V_{R E F},
\end{aligned}
$$

where $C_{V R E F}^{M}$ denotes the total capacitors connected to $V_{R E F}$ in the main-DAC.

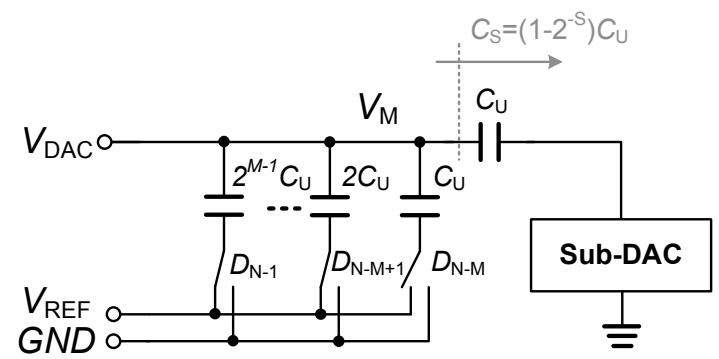

Figure 2.8: A simplified DAC circuit with the whole sub-DAC connected to ground.

Secondly, we consider the case shown in Fig. 2.9, where the entire main-DAC is connected to ground and several capacitors in the sub-DAC is connected to $V_{R E F}$. The voltage at $V_{S}$ is

$$
\begin{aligned}
V_{S} & =\frac{C_{V R E F}^{S}}{\left(2^{S}-1\right) C_{U}+\left(1-2^{-M}\right) C_{U}} V_{R E F} \\
& =\frac{C_{V R E F}^{S}}{2^{S}\left(1-2^{-N}\right) C_{U}} V_{R E F}
\end{aligned}
$$

where $C_{V R E F}^{S}$ denotes the total capacitors connected to $V_{R E F}$ in the sub-DAC. 


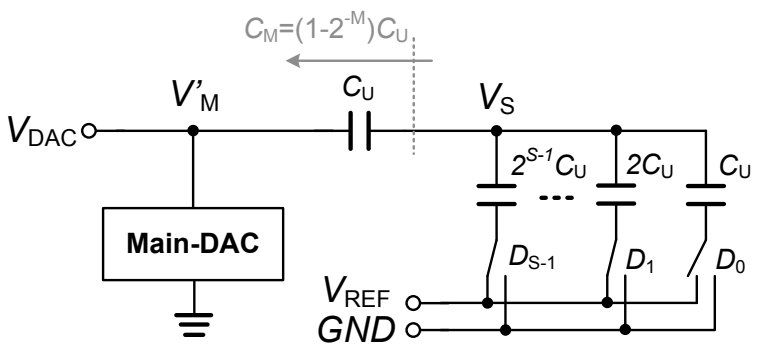

Figure 2.9: A simplified DAC circuit with the whole main-DAC connected to ground.

The series connection of main-DAC capacitors and the bridge capacitor acts as a capacitive divider, hence the voltage at the top-plate of main-DAC, denoted as $V_{M}^{\prime}$, is calculated as

$$
\begin{aligned}
V_{M}^{\prime} & =\frac{C_{U}}{\left(2^{M}-1\right) C_{U}+C_{U}} V_{S} \\
& =\frac{1}{2^{M}} V_{S} .
\end{aligned}
$$

Finally, we can derive the voltage at the DAC output, denoted as $V_{D A C}$, it is

$$
\begin{aligned}
V_{D A C} & =V_{M}+V_{M}^{\prime} \\
& =\frac{C_{V R E F}^{M}}{2^{M}\left(1-2^{-N}\right) C_{U}} V_{R E F}+\frac{1}{2^{M}} \frac{C_{V R E F}^{S}}{2^{S}\left(1-2^{-N}\right) C_{U}} V_{R E F} \\
& =\frac{V_{R E F}}{1-2^{-N}}\left(\frac{C_{V R E F}^{M}}{2^{M} C_{U}}+\frac{C_{V R E F}^{S}}{2^{N} C_{U}}\right) \\
& =\frac{V_{R E F}}{1-2^{-N}} \frac{2^{S} C_{V R E F}^{M}+C_{V R E F}^{S}}{2^{N} C_{U}} .
\end{aligned}
$$

Equation (2.30) shows a gain factor of $1 /\left(1-2^{-N}\right)$. If necessary, the gain error introduced by the modified architecture can be calibrated in the digital domain.

\subsubsection{Mismatch Error}

The resolution of main-DAC $M$ is commonly chosen to be equal to or larger than $N / 2$. Considering the effect of the capacitor mismatch in the sub-DAC is reduced by $1 / 2^{M}$, as indicated in Eq. (2.26), the main-DAC dominates the total mismatch performance. Based on Eq. (2.15), the worst-case standard deviation of DNL for $M$-bit main-DAC is 


$$
\sigma_{D N L, M A X}=\sqrt{2^{M}-1} \frac{\sigma_{u}}{C_{u}} L S B^{\prime},
$$

where $L S B^{\prime}$ is equal to $V_{R E F} / 2^{M}$. Considering the mismatch error should be less than $1 / 2 L S B$, where the LSB is equal to $V_{R E F} / 2^{N}$, we further write

$$
\begin{aligned}
3 \sqrt{2^{M}-1} \frac{\sigma_{u}}{C_{u}} \frac{V_{R E F}}{2^{M}} & <\frac{1}{2} \frac{V_{R E F}}{2^{N}} . \\
\frac{\sigma_{u}}{C_{u}} & <\frac{1}{3 \sqrt{2^{M}-1} \cdot 2^{N-M+1}} .
\end{aligned}
$$

Following a similar method which derives the lower bounds of mismatch-limited unit capacitor for a single binary-weighted capacitive array in Sec. 2.2.1, the lower bounds of mismatch-limited unit capacitor for the modified split architecture is expressed as

$$
C_{U}=18 \cdot\left(2^{M}-1\right) \cdot 2^{2(N-M)} \cdot K_{\sigma}^{2} \cdot K_{C}
$$
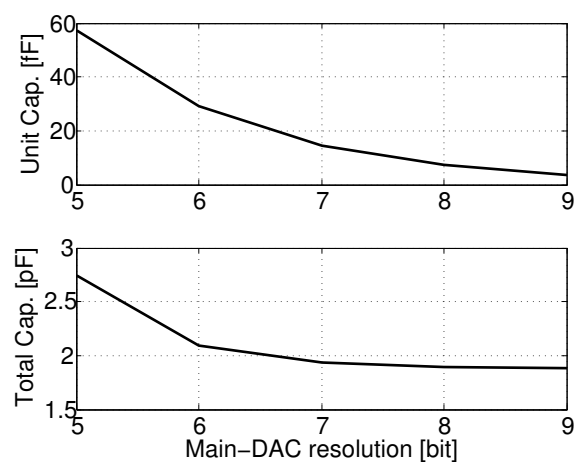

Figure 2.10: Unit capacitance and total array capacitance versus main-DAC resolution.

It will be informative to do a plot based on Eq. (2.34). Assuming 10-bit resolution, $K_{\sigma}=1 \% \mu \mathrm{m}$ and $K_{C}=1 \mathrm{fF} / \mu \mathrm{m}^{2}$, the mismatch-limited minimum unit capacitance together with the corresponding total array capacitance versus main-DAC resolution are plotted in Fig. 2.10. As is shown, the linearity requirements impose much larger unit capacitance and total array capacitance to the split architecture compared with the single architecture.

The actual implementation of the minimum capacitor could be limited by the technology design-kit, denoted as $C_{P R E}$. For a single architecture, a unit capacitance of $C_{P R E}$ might be much larger than necessary to meet with the linearity requirements, resulting in considerably large array capacitance. In this case, a split architecture is 
preferred, which requires larger unit capacitor but still arrives at smaller total array capacitance.

\subsection{Comparator}

Two common approaches to performing the comparison are a latch proceeded by preamplifiers $[18,21]$ or a single dynamic latch $[12,22]$.

\subsubsection{Preamplification Stage}

The preamplification stage usually comprises a single-pole amplifier (SPA) or a cascade of several identical SPAs. As illustrated in Fig. 2.11, the SPA consists of a differential pair, a resistive load, and a bias transistor.

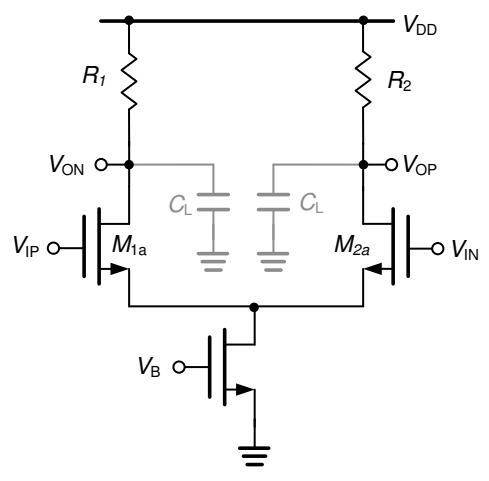

Figure 2.11: A single-pole preamplifier.

\subsubsection{Step Response}

Fig. 2.12 shows the small-signal model of the single-pole amplifier. Assume that a step voltage is applied at the input, the differential output voltage can be expressed as

$$
V_{o}=g_{m a} V_{i} R_{L}\left(1-e^{-t / R_{L} C_{L}}\right),
$$

where $g_{m a}$ is the transconductance of the input transistor. For $t<<R_{L} C_{L}$, the output voltage can be further simplified to

$$
V_{o}=g_{m a} V_{i} R_{L} \frac{t}{R_{L} C_{L}}=t \frac{g_{m a}}{C_{L}} V_{i} .
$$




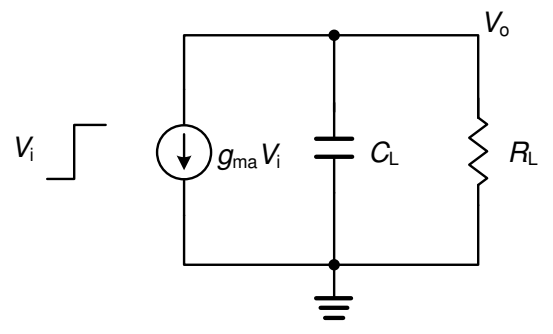

Figure 2.12: Small signal-model of the single-pole preamplifier.

Eq. 2.36 is equal to the step response of an integrator, which only has capacitive load. The load resistor of the SPA shunts current away from the load capacitor, which slows down the amplification.

A multi-stage amplifier, comprising a cascade of several identical SPAs, is a common approach to achieve high gain with relatively high bandwidth. Denoting the DC gain of a single stage as $A_{0}$, the $3-\mathrm{dB}$ bandwidth as $\omega_{0}$, and the total stage as $N$, the equivalent $3-\mathrm{dB}$ bandwidth can be calculated from

$$
\begin{aligned}
\left|A_{N}\left(\omega=\omega_{3 d B}\right)\right| & =\left(\frac{A_{0}}{\sqrt{1+\left(\omega_{3 d B} / \omega_{0}\right)^{2}}}\right)^{N}=\frac{A_{0}^{N}}{\sqrt{2}} . \\
\omega_{3 d B} & =\omega_{0} \sqrt{2^{\frac{1}{N}}-1 .}
\end{aligned}
$$

Since linear amplification is not required in comparison, the output doesn't need to settle close to its steady state at the time the comparison actually takes place [23] Thus, neglecting the load resistor, the step response of a multi-stage amplifier is equal to that of a multi-stage integrator [23]

$$
\begin{aligned}
V_{1} & =\frac{1}{C_{L}} \int_{0}^{t} g_{m a} V_{i} d t=t \frac{g_{m a}}{C_{L}} V_{i} . \\
V_{2} & =\frac{1}{C_{L}} \int_{0}^{t} g_{m a} V_{1} d t=\frac{t^{2}}{2}\left(\frac{g_{m a}}{C_{L}}\right)^{2} V_{i} . \\
\ldots & \\
V_{N} & =\frac{1}{C_{L}} \int_{0}^{t} g_{m a} V_{N-1} d t=\frac{t^{N}}{N !}\left(\frac{g_{m a}}{C_{L}}\right)^{N} V_{i} .
\end{aligned}
$$

Figure 2.13 shows the response time normalized by $C_{L} / g_{m}$ as a function of total number of single stages under two different gains. It can be seen that for a given gain $\left(V_{N} / V_{i}\right)$, there exists an optimum number of stages, $N_{o p}$, for which the response time is minimized. 


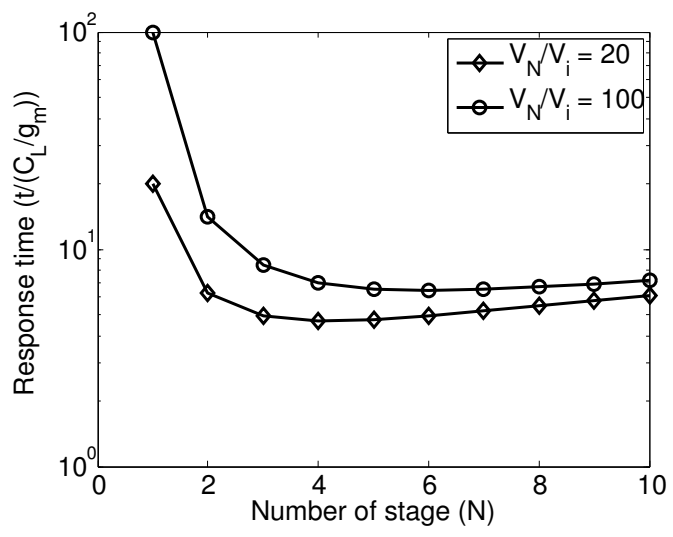

Figure 2.13: Normalized response time of multi-stage amplifier as a function of $N$.

\subsubsection{Offset}

The offset of the amplifier is mainly due to the mismatch of its input transistors. The offset voltage can be approximated to [24]

$$
V_{o s}=\Delta V_{g s}=\Delta V_{t h}+\frac{\Delta \beta}{2 \beta}\left(V_{g s}-V_{t h}\right) .
$$

In practical CMOS technologies, we have [24]

$$
\begin{aligned}
\Delta V_{t h} & =\frac{A_{V t h}}{\sqrt{W L}} t_{o x} \text { with } \\
A_{V t h} & =1.5 \mathrm{mV} \mu \mathrm{m} / \mathrm{nm} \text { and } \\
\frac{\Delta \beta}{\beta} & =1 \%-3 \%
\end{aligned}
$$

\subsubsection{Thermal Noise}

As the drain current noise of the input transistor normally dominates, the input-referred thermal noise of the amplifier is expressed as

$$
v_{i, n}^{2}=\frac{8 k T \gamma B_{n}}{g_{m a}}
$$


where $k$ is the Boltzmann constant, $T$ is the absolute temperature, $\gamma$ is the noise factor, and $B_{n}$ is the noise bandwidth of the amplifier, which can be further expressed as

$$
B_{n}=\frac{1}{4 R_{L} C_{L}} .
$$

Substituting (2.44) into (2.43), (2.43) can be rewritten as

$$
v_{i, n}^{2}=\frac{2 k T \gamma}{C_{L} R_{L} g_{m a}} .
$$

Eq. (2.45) can be further expressed in terms of amplifier gain $A=g_{m a} R_{L}$ or bandwidth $f_{3 d B}=1 / 2 \pi R_{L} C_{L}$

$$
\begin{gathered}
v_{i, n}^{2}=\frac{2 k T \gamma}{A C_{L}} . \\
v_{i, n}^{2}=\frac{4 \pi k T \gamma f_{3 d B}}{g_{m a}} .
\end{gathered}
$$

Assume the noise power is designed to be no larger than the quantization noise $v_{q n}^{2}$, the minimum requirement on gain-load product $A C_{L}$ and $g_{m a}$ are hereafter calculated as

$$
\begin{gathered}
A C_{L}=\frac{2 k T \gamma}{v_{q n}^{2}} . \\
g_{m a}=\frac{4 \pi k T \gamma f_{3 d B}}{v_{q n}^{2}} .
\end{gathered}
$$

\subsubsection{Flicker Noise}

Apart from thermal noise, flicker noise is another important noise source. We start the estimation by referring a known result of flicker noise on the transistor gate [25], which is given by

$$
V_{n F}^{2}=\frac{K_{f}}{C_{g}} \ln \frac{B_{n}}{f_{L}},
$$

where $K_{f}$ is the noise coefficient (on the order of $10^{-25} V^{2} F$ [26]), $C_{g}$ is the gate capacitance, $B_{n}$ is the noise bandwidth, and $f_{L}$ is a lower frequency limit. $B_{n}$ and $f_{L}$ can be further expressed with

$$
B_{n}=\frac{1}{4 R_{L} C_{L}} .
$$




$$
f_{L}=\frac{1}{t_{r s t}}
$$

where $t_{r s t}$ is the reset time of the system. Combining all the above equations, we rewrite Eq. (2.50) as

$$
V_{n F}^{2}=\frac{K_{f}}{C_{g}} \ln \frac{t_{r s t}}{4 R_{L} C_{L}}
$$

\subsubsection{Dynamic Latch}

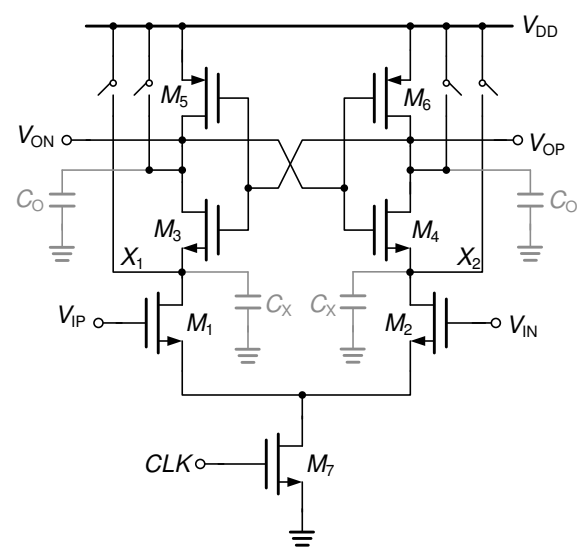

Figure 2.14: A dynamic latch comparator [27].

The regenerative sense amplifier [27], as shown in Fig. 2.14, is a frequentlyadopted latch configuration due to its high power efficiency. The latch works at two modes: reset mode and regeneration mode. When CLK goes low, it is in reset mode, both internal nodes $X_{1} / X_{2}$ and output nodes $V_{O N} / V_{O P}$ are connected to the supply voltage. When CLK goes high, it enters the regeneration mode, which can be further divided into three phases ${ }^{1}$ :

- Phase $1\left(0<t<t_{1}\right)$ : at $t=0$, the current flowing in $M_{1} / M_{2}$ starts to discharge nodes $X_{1} / X_{2}$; at $t=t_{1}$, one of the two nodes first reaches $V_{D D}-V_{T N}$, thus turning on the corresponding NMOS $\left(M_{3} / M_{4}\right)$. $V_{T N}$ is the threshold voltage of the NMOS devices.

- Phase $2\left(t_{1}<t<t_{2}\right)$ : at $t=t_{1}$, the current flowing in $M_{3} / M_{4}$ starts to dischage nodes $V_{O N} / V_{O P}$; at $t=t_{2}$, one of the output nodes first reaches

\footnotetext{
${ }^{1}$ In advanced CMOS nodes, where supply voltage is low and transistor channel is short, the operation region of transistors becomes vague. Thereby compared to [28], which strictly defines the transistor operation region, in this thesis we focus more on the transient evolution of the regeneration.
} 
$V_{D D}-\left|V_{T P}\right|$, thus turning on the corresponding PMOS $\left(M_{5} / M_{6}\right) . V_{T P}$ is the threshold voltage of the PMOS devices.

- Phase $3\left(t>t_{2}\right)$ : the cross-coupled inverters perform regeneration. Finally, one of the outputs is pulled towards ground, and the other up to the supply.

\subsubsection{Step Response}

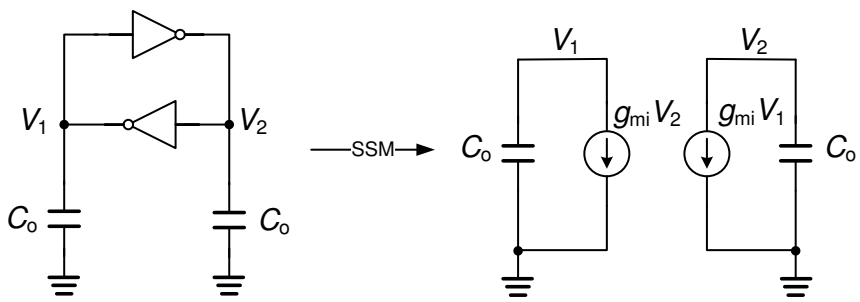

Figure 2.15: Simplified latch circuit and its small-signal model at regeneration mode.

In order to calculate the transient response of the latch at regeneration mode, we simplify the latch circuit to Fig. 2.15. Based on the small-signal model, using Kirchhoff's current law (KCL) for output nodes $V_{1}$ and $V_{2}$, the following coupled first-order differential equations can be obtained

$$
\begin{aligned}
& \frac{d V_{1}}{d t}=-\frac{g_{m i} V_{2}}{C_{o}}, \\
& \frac{d V_{2}}{d t}=-\frac{g_{m i} V_{1}}{C_{o}},
\end{aligned}
$$

where $C_{o}$ is the output load capacitor and $g_{m i}$ is the effective transconductance of the inverter. The differential output in time domain can then be expressed as

$$
V_{1}-V_{2}=\left(V_{1}-V_{2}\right)_{\text {initial }} e^{\frac{t}{C_{o} / g_{m i}}}
$$

It will be interesting to compare the transient response among the three configurations: an SPA, a multi-stage amplifier (MSA), and a latch. The equivalent amplification $U$ for these circuits is defined as the ratio of the differential output to the input step amplitude after an amplification time $t_{a}$ [23]. According to Eq. (2.36), Eq. (2.38), and Eq. (2.56), we rewrite the relationships between $t_{a}$ and $U$ for the SPA, MSA, and the latch

$$
t_{a, S P A}=\frac{C_{L}}{g_{m a}} \times U
$$




$$
\begin{aligned}
t_{a, M S A} & =\frac{C_{L}}{g_{m a}} \times(U \times N !)^{1 / N} . \\
t_{a, \text { latch }} & =\frac{C_{o}}{g_{m i}} \times \ln (U) .
\end{aligned}
$$

Figure 2.16 shows the amplification time, normalized by $C / g_{m}$, as a function of equivalent amplification among an SPA, a 3-stage SPA, and a latch. As expected, the amplification required during comparison is best achieved by the latch.

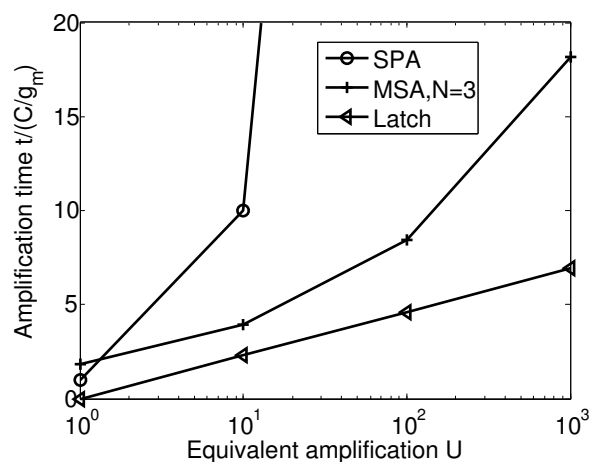

Figure 2.16: Comparison of transient response among SPA, MSA, and latch.

\subsubsection{Offset}

There are mainly two types of offset voltages in the latch: 1) static offset - offset voltage from the mismatch in transistor current factors and in threshold voltages; 2) dynamic offset - offset voltage from the mismatch in the parasitic capacitors.

Since the static offset is quite similar to that of a preamplifier, which has been discussed in Sec. 2.3.1.2, here we look at the dynamic offset which is mainly caused by the mismatch of the load capacitors. The offset voltage introduced by the output load capacitor, $C_{o}$, can be approximated to [29]

$$
V_{o s, c o}=\frac{1}{2} \frac{\Delta C_{o}}{C_{o}}\left(V_{o, 0}-V_{t h i}\right),
$$

where $\Delta C_{o}$ is the absolute capacitance mismatch, $V_{o, 0}$ refers to the initial voltage of $V_{O P}$ before regeneration, and $V_{t h i}$ is the switching voltage of the inverter. The equation shows that the offset voltage is more affected by the relative capacitance mismatch $\left(\Delta C_{o} / C_{o}\right)$ than the absolute capacitance mismatch $\left(\Delta C_{o}\right)$. In [29], it has also been demonstrated that a capacitive imbalance of $1 \mathrm{fF}$ at the output of a simplified latch model (a cross-coupled inverter pair) can lead to offsets of several tens of millivolts. 
In addition, the mismatch of the internal load capacitor, $C_{x}$, at the drain nodes of the input pair will introduce offset. The difference of the capacitance needs to be compensated by a corresponding difference of discharging currents during the initial phase of the latch operation. This difference translates into the amount of offset voltage, which can be approximated to [30]

$$
V_{o s, c x}=\frac{I}{g_{m}} \frac{\Delta C_{x}}{C_{x}},
$$

where $I$ and $g_{m}$ respectively refer to the bias current and transconductance of the input transistor.

\subsubsection{Thermal Noise}

Following the aforementioned analysis, the transient response of the latch at regeneration mode can be re-written as

$$
V_{o}=A_{k} V_{i} e^{t / \tau}
$$

where $V_{o}$ is the output voltage difference, $A_{k}$ is the gain factor before the latch enters Phase $3, V_{i}$ is the input voltage difference, and $\tau$ is the time constant during regeneration. $\tau$ can be calculated from $C_{o} / g_{m i}$.

During Phase 1 and Phase 2, the latch acts as two cascaded integrators [31]. Hence, the corresponding gain can be derived as

$$
A_{k}=\frac{g_{m 1} t_{1}}{C_{x}} \frac{g_{m 3}\left(t_{2}-t_{1}\right)}{C_{o}},
$$

where $g_{m 1}$ is the transconductance of $M_{1}$ in Phase 1 , and $g_{m 3}$ is the transconductance of $M_{3}$ in Phase 2. Further defining a parameter $V_{e f f}$, the bias current of the transistor can be expressed as $I_{D}=g_{m} V_{\text {eff }}$ [32]. Then it is possible to further approximate (2.63) in terms of $V_{\text {eff }}$ and threshold voltages of $M_{1} / M_{3}$ as ${ }^{2}$ :

$$
A_{k}=\frac{V_{T N}}{V_{e f f 1}} \frac{\left|V_{T P}\right|}{V_{e f f 3}} .
$$

Since the latch is reset for each bit cycling, the flicker noise can be substantially reduced. Consequently, the comparator is constrained by thermal noise. Unlike amplifiers, in which operation regions of all the transistors are well-defined, the latch comparators possess time-varying nature, thus making the noise analysis more difficult. In [28], the authors performed noise analysis based on stochastic differential

\footnotetext{
${ }^{2}$ In practice, the device starts to conduct even the gate-source voltage is less than its threshold voltage. This indicates the regeneration may start before the circuit enters Phase 3, thus leading to a smaller effective gain factor compared to Eq. (2.64).
} 
equations; in [31], the authors estimated the comparator decision error probability due to thermal noise based on linear, periodically time-varying systems. Based on the results from $[28,31]$, we simplify the expression of latch output noise as

$$
V_{l o, n}^{2}=\kappa_{1} \frac{k T}{C_{o}}+\kappa_{2} \frac{\gamma k T}{C_{o}}
$$

where $\kappa_{1}$ and $\kappa_{2}$ are architecture-dependent parameters. The first and second term in the right-hand-side of Eq. (2.65) respectively correspond to the thermal noise contributed from the reset transistors and the active transistors. With $\kappa_{1}=\kappa_{2}=2$, Eq. (2.65) becomes exactly the output noise of a simple latch comprising a crosscoupled inverter [33]. The input-referred thermal noise of the latch comparator is then equal to Eq. (2.65) divided by $A_{k}^{2}$ [33]. If pre-amplification stage is proceeded, then the latch thermal noise can be further divided by the power of the pre-amplification gain.

\subsubsection{Metastability}

Metastability is the phenomenon where a bistable element requires an indeterminate amount of time to generate a valid output [34]. The metastability in a latch comparator occurs when the differential input signal is so small that the latch does not have enough time to produce a well-defined logic level, which might be interpreted differently by succeeding logic, leading to conversion errors.

Recall Eq. (2.62) and copy as follows

$$
V_{o}=A_{k} V_{i} e^{t / \tau}
$$

Assume that the acceptable logic level (trip point) for $V_{o}$ is $V_{D D} / 2$, otherwise, metastable outputs will be caused. Based on the allowable comparator decision time, denoted as $T_{\max }$, the minimum required input voltage difference can be expressed as

$$
V_{i, M I N}=\frac{1}{A_{k}} \frac{V_{D D}}{2} e^{-T_{\max } / \tau} .
$$

Further assume that the input signal follows a uniform distribution across a voltage range $V_{M}$. The probability of metastable error $p_{M}$ is equal to the probability when the input voltage difference is less than $V_{i, M I N}$. We write

$$
\begin{aligned}
p_{M} & =P\left(\left|V_{i}\right|<V_{i, M I N}\right) \\
& =2 \times \frac{V_{i, M I N}}{V_{M}} \\
& =\frac{1}{A_{k}} \frac{V_{D D}}{V_{M}} e^{-T_{\max } / \tau} \quad \text { (errors/sample). }
\end{aligned}
$$




\section{Chapter 3}

\section{SAR ADC Power Analysis}

A deep understanding of the ADC power bounds helps to further reduce its power consumption. The power bounds of SAR ADC are analyzed based on the factors which will degrade the conversion resolution, such as noise and mismatch. As we are looking for the lower power bounds, we have limited our study to power-efficient SAR ADC architectures, such as a charge-redistribution SAR ADC [35]. As shown in Fig. 3.1, the ADC consists of a binary-weighted capacitive array, a dynamic latch comparator, and a SAR control logic. The capacitive DAC samples the input and generates approximations of the input based on the converted digital value. The comparator compares approximations to the sampled input, serially determining output bits. The SAR control logic performs the successive approximation algorithm and drives the switches in the capacitive DAC. The power consumption of each of these blocks is analyzed in this chapter.

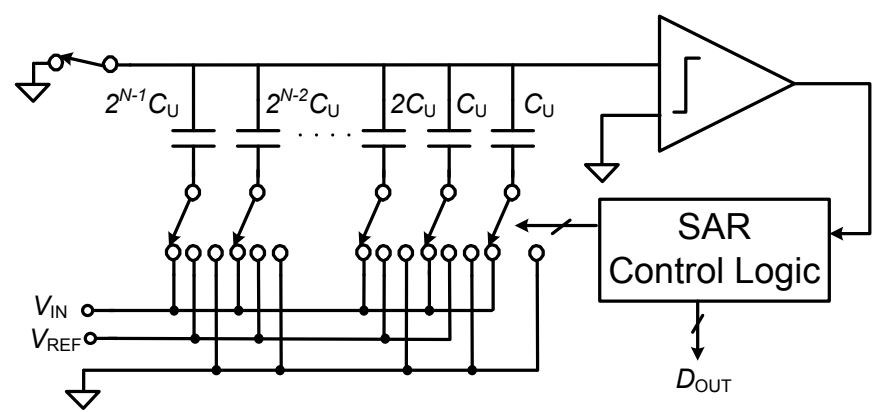

Figure 3.1: Charge-redistribution SAR ADC [35]. 


\subsection{Capacitive DAC}

Assume the input signal sampled on the DAC is uniformly distributed between ground and the reference voltage $V_{R E F}$, the average switching power of the DAC depends on its array capacitance and employed switching approach. For an $N$-bit capacitive DAC employing a conventional charge-recycling switching approach [35], the average switching power is derived as [36]

$$
P_{D A C}=0.66 \times 2^{N} f_{s} C_{u} V_{R E F}^{2} .
$$

To reduce the power, the unit capacitor should be as small as possible. In practice, however, it is usually determined by thermal noise and capacitor mismatch. The thermal noise resulting from the track-and-hold action is given by the well-known equation: $k T / C$, where the capacitance in this case is equal to the total array capacitance, $2^{N} C_{u}$. For a Nyquist $\mathrm{ADC}, C_{u}$ is typically chosen large enough so that the thermal noise is less than the converter's quantization noise $v_{q n}^{2}$, which thereby gives a minimum value of $C_{u}$

$$
C_{u, n}=\frac{k T}{2^{N} v_{q n}^{2}} .
$$

The capacitor mismatch degrades the linearity of the ADC, commonly specified in terms of differential nonlinearity (DNL) and integral nonlinearity (INL). Both analog calibration and digital error correction have been proposed to deal with the mismatch problem: the former uses additional capacitor banks to calibrate the capacitors in the foreground [37]; the latter utilizes equalization techniques to extract the exact weights of the capacitive array in the background [38, 39]. With these methods, the matching requirement in the capacitive array becomes more relaxed but at the cost of design complexity. In this analysis, we study the power cost of performing mismatch compensation by simply sizing the capacitor large enough so that expected statistical deviation meets the mismatch limit for the desired ADC linearity. In Sec. 2.2.1, we have derived the mismatch-limited minimum $C_{u}$. For ease of reference, we copy Eq. (2.20) here

$$
C_{u, m}=18 \cdot\left(2^{N}-1\right) \cdot K_{\sigma}^{2} \cdot K_{C}
$$

Apart from the above two limiting factors, the process will also set a lower limit to the capacitance so that the total array capacitance at least need to be equal to the parasitic capacitance at the DAC output, which results in a $50 \%$ attenuation of the output voltage. The parasitic capacitance include both the gate capacitance of the comparator input and the parasitic capacitance of interconnection. We further assume it is comparable to the input capacitance of a minimum-sized inverter, which is denoted as $C_{i n v}$. Finally, $C_{u}$ in Eq. (3.1) can be replaced with

$$
C_{u}=\max \left(C_{u, n}, C_{u, m}, C_{i n v}\right)
$$




\subsection{Single-Pole Amplifier}

As illustrated in Fig. 2.11 of Sec. 2.3, the SPA consists of a differential pair, a resistive load, and a bias transistor. Denoting the bias current of the input transistor as $I_{D}$ and the supply voltage as $V_{D D}$, the power consumption of the amplifier is written as

$$
P_{A M P}=2 I_{D} V_{D D}
$$

Defining a parameter $V_{\text {effa }}$, the bias current of the transistor can be expressed as $I_{D}=g_{m a} V_{e f f a}$ [32]. Therefore, Eq. (3.5) can be further written as

$$
P_{A M P}=2 g_{m a} V_{e f f a} V_{D D},
$$

where $g_{m a}$ is the transconductance of the input transistor, and $V_{e f f a}=I_{D} / g_{m a}$. As the drain current noise of the input transistor normally dominates, the input-referred thermal noise of the amplifier is expressed as

$$
v_{i, n}^{2}=\frac{8 k T \gamma B_{n}}{g_{m a}},
$$

where $k$ is the Boltzmann constant, $T$ is the absolute temperature, $\gamma$ is the noise factor, and $B_{n}$ is the noise bandwidth of the amplifier. Assume the noise power is designed to be no larger than the quantization noise $v_{q n}^{2}$, the minimum requirement on $g_{m a}$ is hereafter calculated as

$$
g_{m a}=\frac{8 k T \gamma B_{n}}{v_{q n}^{2}} .
$$

The noise bandwidth $B_{n}$ can be expressed in terms of time constant $\tau$

$$
B_{n}=\frac{\pi}{2} f_{3 d B}=\frac{1}{4 \tau} .
$$

For an $N$-bit SAR ADC, the clock frequency is typically $N+1$ times higher than the sampling frequency (one cycle for sampling and $N$ cycles for bit decisions). To ensure enough settling time (e.g. $7 \tau$ [40]) for the amplifier within half of the clock cycle, the time constant must be no larger than

$$
\tau \leq \frac{1}{7 \times 2 \times f_{C L K}}=\frac{1}{14(N+1) f_{s}} .
$$

Substituting (3.8) into (3.6), and further combining (3.9) and (3.10) into (3.6), the minimum power consumption of the amplifier is calculated as

$$
P_{A M P}=\frac{56(N+1) k T \gamma f_{s} V_{e f f a} V_{D D}}{v_{q n}^{2}} .
$$




\subsection{Latch Comparator}

The schematic of the latch comparator is shown in Fig. 2.14 of Sec. 2.3. A typical signal transient behavior of the differential outputs and the supply current of the latch is visualized in Fig. 3.2.

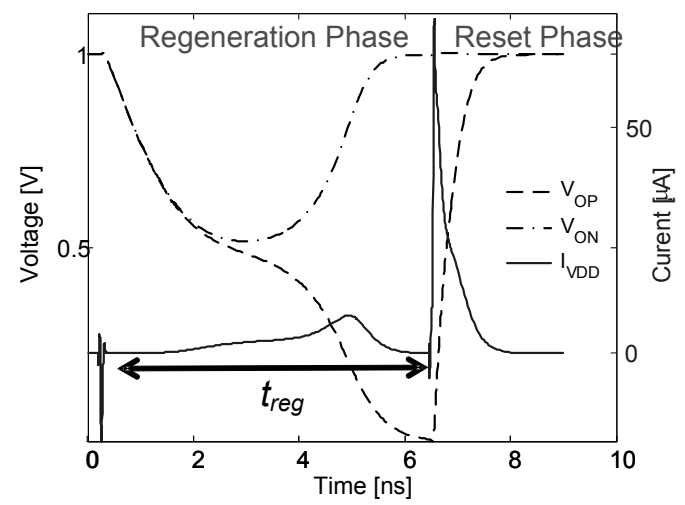

Figure 3.2: Typical signal transient behavior including the differential outputs and the supply current. Note that there is no static supply current.

To compute the charge during the regeneration mode, we denote that there is a current, $I_{D}$, flowing only during the regeneration time, $t_{r e g}$. Hence, the total regenerative charge can be expressed as $2 I_{D} t_{\text {reg }}$. The regeneration time can be calculated from Eq. (2.62). For ease of reference, we copy Eq. (2.62) as follows

$$
V_{o}=A_{k} V_{i} e^{t / \tau}
$$

where $V_{o}$ is the output voltage difference, $A_{k}$ is the gain factor before the latch enters Phase 3, $V_{i}$ is the input voltage difference, and $\tau$ is the time constant during regeneration. $\tau$ can be calculated from $C_{o} / g_{m i}$.

Further defining a parameter $V_{\text {effi }}$, we can write $g_{m i}=I_{D} / V_{\text {effi }}$ [32]. Assuming that the regeneration is finished when $V_{o}$ becomes $V_{D D} / 2$. It results in the following expression of $t_{r e g}$

$$
t_{r e g}=\frac{V_{e f f i} C_{o}}{I_{D}} \ln \frac{V_{D D}}{2 A_{k} V_{i}} .
$$

Using Eq. (3.13), we can rewrite the expression of the regenerative charge for one conversion step as

$$
Q_{C, r e g-s}=2 V_{e f f i} C_{o} \ln \frac{V_{D D}}{2 A_{k} V_{i}}
$$


Since an $N$-bit SAR ADC needs $N$ steps to complete one conversion, the input voltage difference of the comparator for the $i$ th-step can be expressed as

$$
V_{i}(i)=-V_{I N}+\sum_{j=i+1}^{N-1} D_{j} \frac{V_{R E F}}{2^{N-j}}+\frac{V_{R E F}}{2^{i}}, 1 \leq i \leq N
$$

where $V_{I N}$ is the input voltage, $D_{j}$ is the bit decision.

Assume that $V_{I N}$ is evenly distributed between 0 and $V_{R E F}, V_{i}$ is thereby evenly distributed between 0 and a binary-weighted value of $V_{R E F}$, which is denoted as $V_{m}$. Then, the average charge for one step can be expressed by

$$
\frac{1}{V_{m}} \int_{0}^{V_{m}} Q_{C, r e g-s} \mathrm{~d} V_{i}=2 V_{e f f i} C_{o}\left(\ln \frac{V_{D D}}{2 A_{k} V_{m}}+1\right) .
$$

Hence, the charge of a complete conversion can be derived from the total charge of $N$-steps

$$
\begin{aligned}
Q_{C, r e g} & =\sum_{j=1}^{N}\left(2 V_{e f f i} C_{o}\left(\ln \frac{V_{D D}}{2 A_{k}\left(V_{R E F} / 2^{j}\right)}+1\right)\right) \\
& =2 N C_{o} V_{e f f i}\left(\ln \frac{V_{D D}}{2 A_{k} V_{R E F}}+\frac{N+1}{2} \ln 2+1\right) .
\end{aligned}
$$

As regards the reset charge, it is mainly consumed by the capacitive load at the comparator output. Consequently, the total power consumption of the comparator is equal to the reset charge at the clock frequency and the regenerative charge at the sampling frequency

$$
P_{L C}=N f_{s} V_{D D} Q_{C, r s t}+f_{s} V_{D D} Q_{C, r e g} .
$$

By replacing $Q_{C, r s t}$ with $C_{C} V_{D D}$ and $Q_{C, r e g}$ with Eq. (3.18), Eq. (3.19) is rewritten as

$$
P_{L C}=N f_{s} C_{o} V_{D D}^{2}+2 N f_{s} C_{o} V_{e f f i} V_{D D}\left(\ln \frac{V_{D D}}{2 A_{k} V_{R E F}}+\frac{N+1}{2} \ln 2+1\right) .
$$

The random offset of the latch is closely related to its input common-mode voltage. If the common-mode voltage is fixed during conversion, the latch offset usually appears as a constant value and causes the shift of the entire transfer curve. Here, we assume a conventional DAC switching scheme [35], generating a constant common-mode voltage during bit-cycling, so that the latch offset introduces ADC offset rather than nonlinearities. Hence, the fundamental limitation on the achievable comparator resolution is noise. Since the latch is reset for each bit cycling, the flicker noise can be substantially reduced. Consequently, the comparator is constrained 
by thermal noise, which is derived by Eq. (2.65). Equalizing the input-referred thermal noise to the quantization noise of an $N$-bit converter gives a minimum load capacitance

$$
C_{o, n}=\frac{k T\left(\kappa_{1}+\kappa_{2} \gamma\right)}{A_{k}^{2} v_{q n}^{2}} .
$$

Considering that the effect of the process also set a lower limit to the capacitance through minimum feature size. We therefore include $C_{i n v}$, the input capacitance of a minimum-sized inverter. And $C_{o}$ in Eq. (3.20) can be replaced with

$$
C_{o}=\max \left(C_{o, n}, C_{i n v}\right) \text {. }
$$

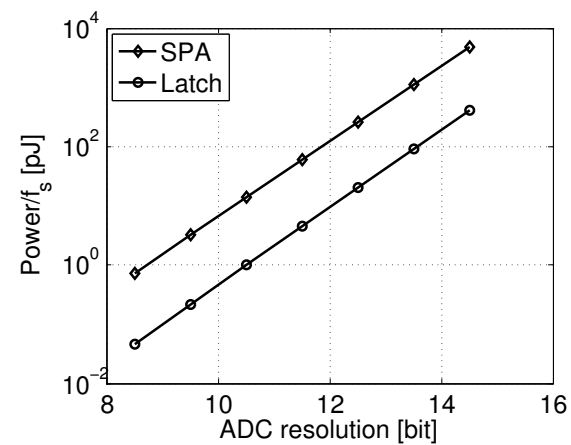

Figure 3.3: Comparison of energy consumption between SPA and latch.

The energy consumption between an SPA and a latch compartor is compared in Fig. 3.3. It can be seen that SPA is less power-efficient. Therefore, only the power from a single latch comparator is included in the total power calculation. However, in practice pre-amplification stages are effective in cases where latch noise needs to be reduced or latch metastability error to be mitigated.

\subsection{SAR Control Logic}

A straightforward way to build a SAR control logic is to use a ring counter and a shift register, resulting in $2(N+1)$ D-type Flip-Flops (DFFs) for $N$-bit resolution, as shown in Fig. 3.4. Among different DFF design styles, the pass-gate style, as shown in Fig. 3.5, is one of the low-power configurations [41]. The DFF comprises 2 cross-coupled inverter pairs for data path; 2 transmission gates and 2 inverters for clock path. Assuming the capacitive load of one DFF is equivalent to that of 8 inverters, the equivalent capacitive load of the SAR logic can be approximated to that 
of $16(N+1)$ inverters in total. Assuming a total activity of the SAR logic to be $\alpha$, then we derive the dynamic power consumption of the SAR logic as

$$
P_{S A R}=16 \alpha(N+1)^{2} f_{s} C_{i n v} V_{D D}^{2},
$$

where $C_{i n v}$ is the input capacitance of a minimum-sized inverter in a particular technology. Note that the SAR logic works at a frequency of $(N+1) f_{s}$.

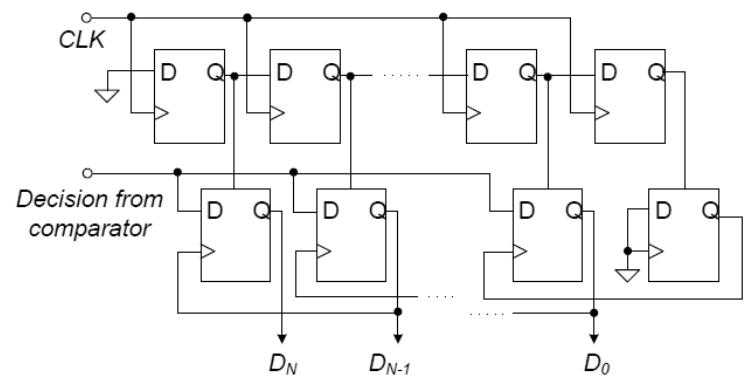

Figure 3.4: A typical design of SAR control logic.

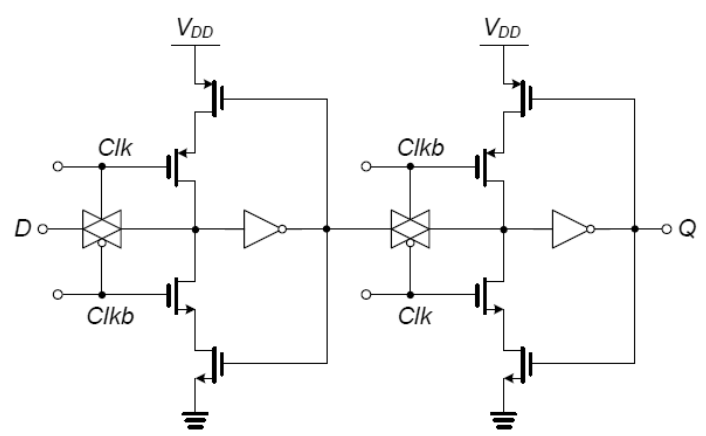

Figure 3.5: A DFF with pass-gate style.

Leakage power consumption could be significant for a low-speed circuit designed in a general-purpose standard CMOS process. However, due to its heavily processand temperature-dependent feature, for simplicity, this portion is neglected in the analysis. 


\subsection{Power Consumption Bounds of an Entire ADC}

Adding together the DAC power Eq. (3.1), the latch comparator power Eq. (3.20), and the SAR logic power Eq. (3.23), the power consumption of an entire SAR ADC can be expressed as

$$
\begin{aligned}
P_{A D C} & =0.66 \times 2^{N} f_{s} C_{u} V_{R E F}^{2}+N f_{s} C_{o} V_{D D}^{2} \\
& +2 N f_{s} C_{o} V_{e f f i} V_{D D}\left(\ln \frac{V_{D D}}{2 A_{k} V_{R E F}}+\frac{N+1}{2} \ln 2+1\right) \\
& +16 \alpha(N+1)^{2} f_{s} C_{i n v} V_{D D}^{2} .
\end{aligned}
$$

For ease of reference, below is a list of all the parameters used in the power model.

$A_{k}$ Gain factor of the latch before it starts regeneration

$C_{o}$ Capacitive load of the latch

$C_{u}$ DAC unit capacitance

$C_{i n v}$ Input capacitance of a minimum-sized inverter

$f_{s}$ Sampling frequency

$K_{c}$ MIM capacitor density parameter

$K_{\sigma}$ MIM capacitor matching coefficient

$T$ Temperature

$V_{D D}$ Supply voltage of the ADC

$V_{\text {eff }}$ Effective voltage of a transistor $\left(\equiv I_{D} / g_{m}\right)$

$V_{R E F}$ Reference voltage of the ADC

$V_{T N}$ Threshold voltage of NMOS devices

$V_{T P}$ Threshold voltage of PMOS devices

$v_{q n}^{2}$ Quantization noise power

$\alpha$ Switching activity of the SAR logic

$\gamma$ Thermal noise factor

$\kappa_{1} \kappa_{2}$ Architecture-dependent parameters for output thermal-noise of a latch comparator 


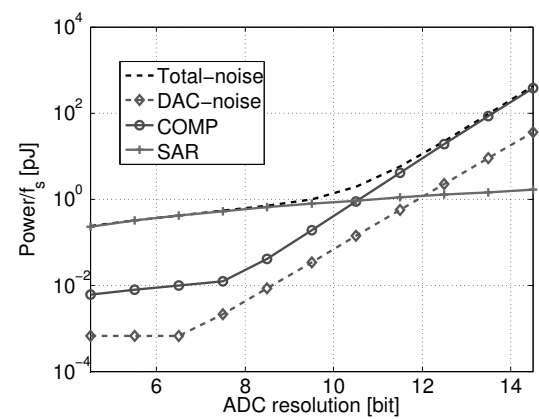

(a)

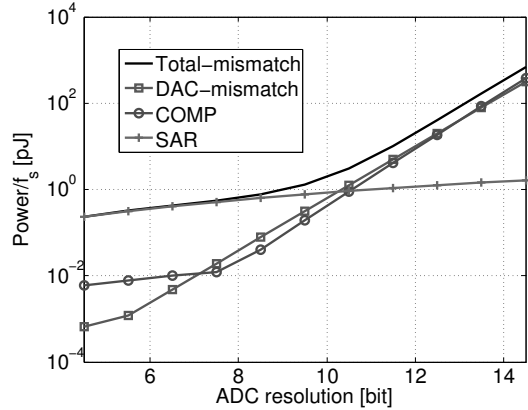

(b)

Figure 3.6: Analyzed energy bounds of an entire ADC with its individual blocks: (a) DAC is noise-limited (b) DAC is mismatch-limited.

Figure 3.6 shows the analyzed energy bounds of an entire SAR ADC together with its individual blocks. We plot the DAC power consumption limited by noise and mismatch, respectively. When the resolution is low, the digital logic dominates the total power. However, for medium to high resolution, the total power is close to the sum of DAC and comparator power, if mismatch is a limiting factor. When mismatch can be calibrated and thermal noise becomes the limiting factor, the comparator power dominates the total power.

Table 3.1: Parameter Values Used in Demonstration

\begin{tabular}{|c|c|c|}
\hline$C_{i n v}=1 f F$ & $K_{c}=1 f F / \mu m^{2}$ & $K_{\sigma}=0.5 \% \mu m$ \\
\hline$T=300 K$ & $V_{D D}=1 V$ & $V_{e f f a}=50 m V$ \\
\hline$V_{e f f[i, 1,3]}=0.15 V$ & $V_{R E F}=1 V$ & $V_{T N}=0.3 V$ \\
\hline$V_{T P}=0.4 V$ & $\alpha=0.4$ & $\gamma=2$ \\
\hline$\kappa_{1}=\kappa_{2}=2$ & & \\
\hline
\end{tabular}

Table 3.1 shows the parameter values used in the demonstration. We assume the $\mathrm{ADC}$ works at room temperature with a full-scale input voltage equal to the supply of $1 \mathrm{~V}$. Typical values for the threshold voltage of transistors are used: $0.3 \mathrm{~V}$ and $0.4 \mathrm{~V}$ for NMOS and PMOS devices, respectively. The thermal noise factor is equal to $2 / 3$ for long-channel transistors and a larger value, 1.5 - 3, for submicron transistors [42]. Here, $\gamma$ is set to be 2. By referring to [28, 31, 33], both $\kappa_{1}$ and $\kappa_{2}$ are assigned to be 2 . The density parameter and the matching coefficient of the MIM capacitors are chosen to be $1 \mathrm{fF} / \mu m^{2}$ and $0.5 \% \mu m$ [43, 44], respectively.

Since half of the transistors in a DFF are controlled by the clock signal and half of DFFs in the SAR logic are clocked with full activity, thus one-fourth of the inverters in the SAR logic have a switching activity of 1 . Assume the rest three-fourth of the 
inverters has a relatively low activity of 0.2 , the switching activity $\alpha$ is calculated to be 0.4 .

Ideally $C_{i n v}$ is about 4 times larger than the minimum NMOS gate capacitance (the PN ratio of the inverter is assigned to be 3 ). The simulated $C_{i n v}$ in $65-\mathrm{nm}$ technology is about $0.85 \mathrm{fF}$. Including some margins for wire capacitance, $C_{i n v}$ is assumed to be equal to $1 \mathrm{fF}$.

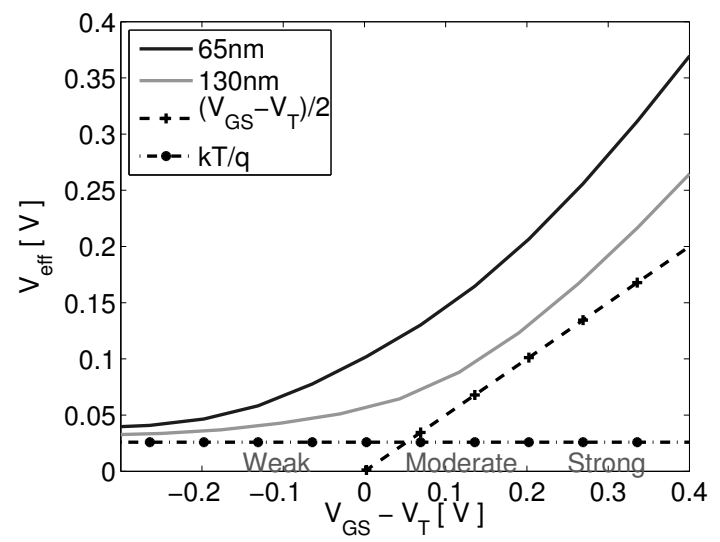

Figure 3.7: $V_{e f f}$ versus $V_{G T}$ for minimum-sized NMOS transistor in 65-nm and 130-nm technologies.

High power efficiency of amplifier corresponds to operating the transistors in moderate to weak inversion, thereby resulting in low $V_{e f f}$, as shown in Fig. 3.7. Hence, the effective voltage of the input transistor in the preamplifier, $V_{\text {effa }}$, is chosen to be $50 \mathrm{mV}$, about two times of the thermal voltage $(k T / q)$.

The rest of the parameters are the effective voltages of the transistors in the latch: $V_{\text {eff } 1}, V_{\text {eff } 3}$, and $V_{\text {eff } i}$. Different from the transistors in the preamplifier, where their operation regions can be controlled by the bias current, the transistors in the latch undergo time-varying operation regions. Considering that the transistors in the latch start from strong inversion, which corresponds to effective voltages larger than $0.1 \mathrm{~V}$ in Fig. 3.7, we thereby choose a relatively higher effective voltage of $0.15 \mathrm{~V}$. And for simplicity, we assign the three to be the same value. According to Eq. (2.64), the gain factor of the latch is calculated to be 5 . 


\section{Chapter 4}

\section{Design of 10-bit 1-kS/s SAR ADCs}

In this chapter, two 10-bit 1-kS/s SAR ADCs for medical implant devices are presented. The first ADC is a 53-nW 9.1-ENOB SAR ADC implemented in $0.13 \mu \mathrm{m} \mathrm{CMOS}$ process. To achieve the nano-watt range power consumption, an ultra-low power design strategy has been utilized, imposing maximum simplicity on the ADC architecture, low transistor count and matched capacitive DAC with a switching scheme which results in full-range sampling without switch bootstrapping and extra reset voltages. Furthermore, a dual-supply voltage scheme allows the SAR logic to operate at $0.4 \mathrm{~V}$, reducing the overall power consumption of the ADC by $15 \%$ without any loss in performance.

Based on the understanding from the first $\mathrm{ADC}$ and motivated by the predicted power bounds, the second ADC, a 3-nW 9.1-ENOB SAR ADC in 65nm CMOS process was implemented with a substantial (94\%) improvement in the power consumption. Taking advantage of the smaller feature size and the availability of standardand high- $\mathrm{V}_{\mathrm{T}}$ devices in $65 \mathrm{~nm}$ process, the ultra-low-power consumption is achieved by: 1) a split-array capacitive DAC, which substantially reduces the DAC capacitance while still ensuring sufficient linearity; 2) a bottom-plate sampling approach reducing the charge injection error due to use of small DAC capacitance, while enabling fullrange input sampling without extra voltage sources; 3 ) a multi- $\mathrm{V}_{\mathrm{T}}$ design, allowing the ADC to meet the target performance with a single supply of $0.7 \mathrm{~V}$, thereby reducing both the switching and leakage power consumption; and 4) a latch-based SAR control logic resulting in reduced power consumption and low transistor count. 


\subsection{A 53-nW 9.1-ENOB SAR ADC in 0.13 $\mu \mathrm{m}$ CMOS Process}

\subsubsection{Introduction}

Medical implant devices, such as pacemakers and implantable cardiac defibrillators, are to be implanted in the human body and require extremely low power consumption in order to operate up to 10 years or more [45]. As shown in Fig. 1.3 of Chapter 1, the ECG signal covers a frequency band with the highest frequency less than $1 \mathrm{kHz}$. Trading speed for lower power consumption at such slow sampling rate is not a straightforward task. The major challenge is how to efficiently reduce the unnecessary speed and bandwidth for ultra-low-power operation using inherently fast devices in advanced CMOS technologies. Moreover, the leakage current degrades the sampling accuracy during the long conversion time, and the leakage power contributes to a significant portion of the total power consumption. As an example, Fig. 4.1 shows the average power consumption of an inverter as a function of its switching frequency. The power consumption was simulated at two different supplies $(1.0 \mathrm{~V}$ and $0.4 \mathrm{~V})$ over two different sizes $\left(\mathrm{W}_{\min } / \mathrm{L}_{\min }\right.$ and $\left.\mathrm{W}_{\min } / 2 \mathrm{~L}_{\min }\right)$. It can be seen that the leakage power at $1-10 \mathrm{kHz}$ can constitute more than $50 \%(50 \%$ at $10 \mathrm{kHz})$ of the total power.

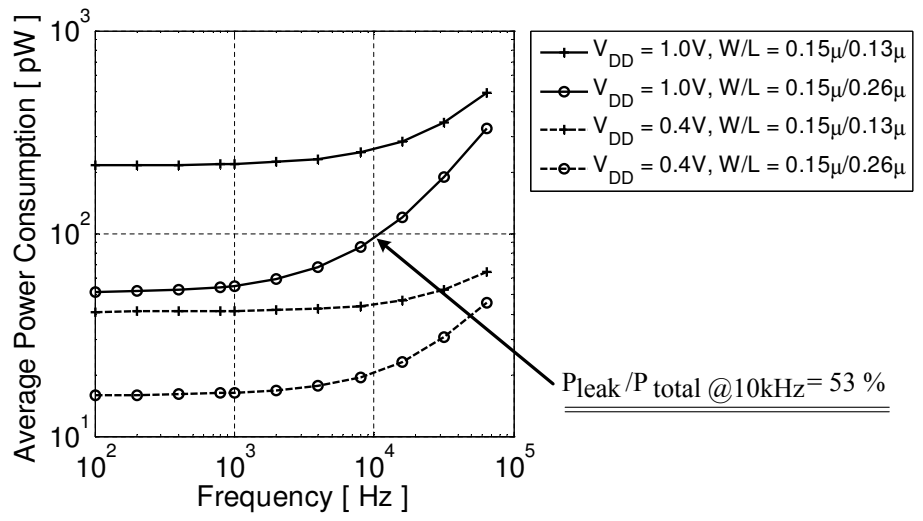

Figure 4.1: Simulated average power consumption versus switching frequency of an inverter with a fan-out of four in $0.13-\mu \mathrm{m}$ CMOS process.

Considering the above discussion and the fact that every nano-watt counts for such ADCs, the main key to achieve the ultra-low-power operation turns out to be the maximal simplicity in the ADC architecture and low transistor count. This essentially means that we avoid ADC techniques with additional complexity and circuit overhead, which are useful for higher sampling rates and resolutions. Taking advantage of 
the low speed, the proposed ADCs utilize matched capacitive DACs, being sized to achieve the targeted conversion accuracy without digital error correction or calibration, thus eliminating additional devices and significant leakage currents. Moreover, the matched capacitive DACs use switching schemes that allow full-range input sampling without additional voltage sources. Compared to the energy-efficient switching schemes [46][47][18], the employed approach introduces less overhead in the SAR control logic [46][18] and avoid additional bias voltages in the comparator [47]. To further reduce the power consumption, a dual-supply voltage scheme was employed, allowing the SAR logic to operate at $0.4 \mathrm{~V}$. Utilizing the above design strategy combined with low-leakage circuit techniques, careful circuit optimizations, and circuit layout, the SAR ADC consumes 53-nW power at a sampling rate of $1 \mathrm{kS} / \mathrm{s}$, achieving 9.1 ENOB.

\subsubsection{ADC Architecture}

Figure 4.2 shows the block diagram of the proposed ADC. It comprises a matched binary-weighted capacitive DAC, a low-power dynamic latch comparator, a lowleakage/low-voltage synchronous SAR digital logic, and level shifters between the digital logic and the analog blocks. In addition, a differential architecture was employed to have a good common-mode noise rejection.

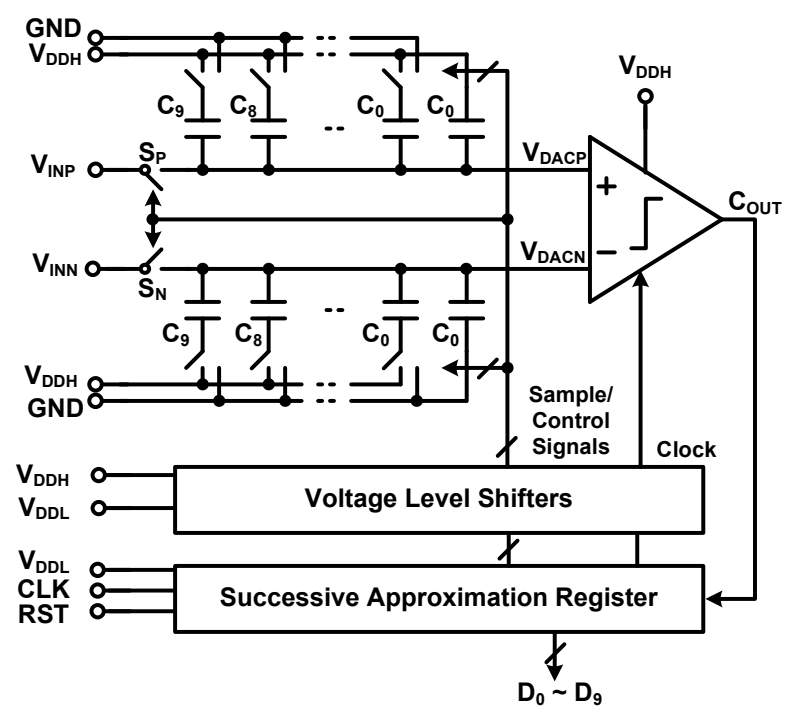

Figure 4.2: Architecture of the SAR ADC. 
In a conventional SAR ADC [35], the input voltage is sampled on the bottom-plate nodes of the capacitor array and the top-plate nodes are reset with a fixed voltage. The fixed voltage is commonly chosen to be one of the power rails in order to avoid extra voltage levels. However, this makes the DAC outputs go beyond the rails during the conversion when full-range input sampling is applied. One common way to solve this problem is to decrease the input range with the penalty of degrading the signal-tonoise ratio. Another alternative is to make the top-plate switches bootstrapped. In this work, we use top-plate sampling [46] with MSB preset to achieve full-range sampling without switch bootstrapping and extra reset voltages. As shown in Fig. 4.3, the differential inputs are initially connected to the top-plates of the capacitor array, and simultaneously the MSB is reset to high and all other bits are reset to low. Next, the top-plate sampling switch is open and the input data is sampled on the capacitor array. The comparator then performs the first comparison. If $V_{D A C P}$ is higher than $V_{D A C N}$, the MSB remains high. Otherwise, it goes low. Then, the second approximation step starts by setting MSB-1 to high, and the comparator does the comparison again. The ADC repeats this procedure until all 10 bits are decided. During the entire conversion, the DAC outputs always remain within the rails. Moreover, the commonmode voltage of the DAC outputs is the same as that of the differential inputs, which is equal to mid-rail voltage for full-range input sampling, as shown in Fig. 4.4. The constant common-mode voltage reduces the signal-dependent offset voltage of the comparator [47].

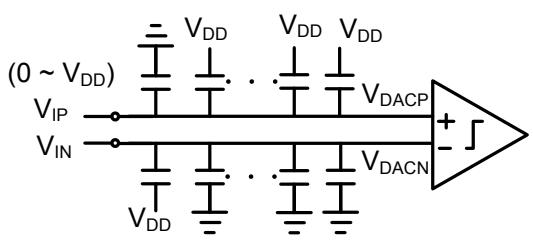

Sampling Phase

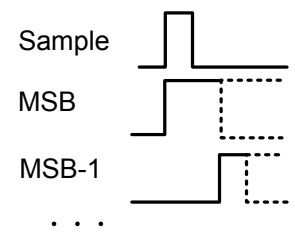

Related Time Sequence

Figure 4.3: The sampling phase of capacitive DAC with MSB preset.

Lowering the supply voltage is an efficient technique to reduce both the switching and leakage power consumption. This is particularly true at low data-rates, where transistors can be slow but still meet the target speed. However, for the analog circuits operating with low supply voltages, noise and a reduced dynamic range can degrade the ADC performance. To avoid the analog performance degradation, in this design, we use a dual-supply voltage scheme, which allows the SAR logic to operate at low supply voltages. Our measurement results (in Sec. 4.1.7) show that this voltage scaling has reduced the overall power consumption of the ADC by $15 \%$ without any loss in performance. 


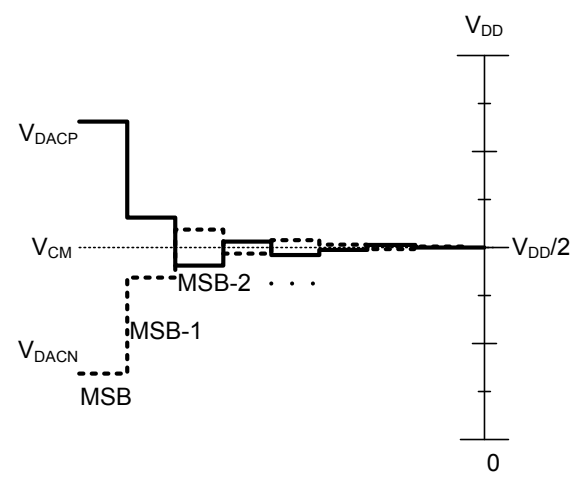

Figure 4.4: Waveform of the DAC switching procedure.

\subsubsection{Capacitive DAC}

The capacitive DAC was implemented with a binary-weighted capacitor array. In this technology, a MIM capacitor has a density of $2 \mathrm{fF} / \mu \mathrm{m}^{2}$ and a matching of $1 \% \mu \mathrm{m}$. Eq. (2.20), which calculates the lower bounds for the mismatch-limited unit capacitor, leads to a minimum unit capacitance of $4 \mathrm{fF}$. Apart from the mismatch, the design rule will also set a minimum value on the MIM capacitance, which is $27 \mathrm{fF}$ in this process. Consequently, the unit capacitance was set to be $13.5 \mathrm{fF}$ in our work, which was implemented by two minimum process-defined MIM capacitors in series. Hence, the total array capacitance is about $14 \mathrm{pF}$.

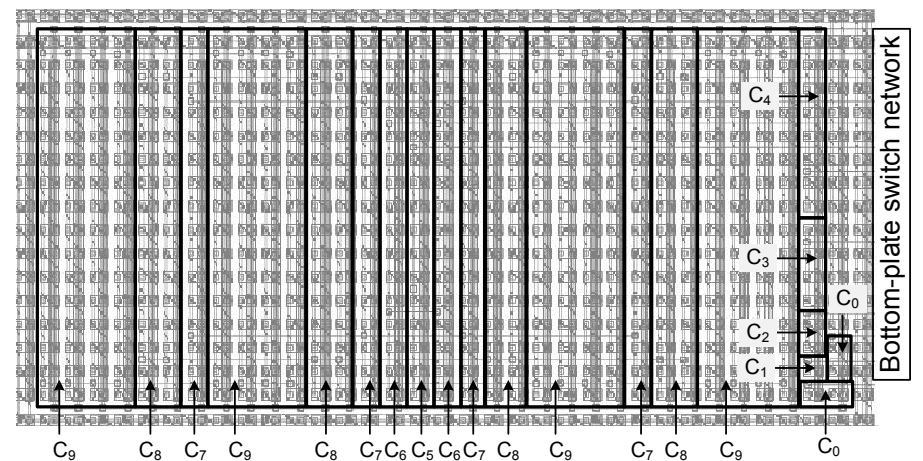

Figure 4.5: Layout of the capacitor array which follows a partial common-centroid configuration. The capacitors are indicated according to Fig. 4.2.

Besides capacitor sizing, a careful layout to avoid linearity degradation is important as well. In this work, we have utilized a partial common-centroid layout 
strategy for the capacitor array. Fig. 4.5 illustrates the layout floor plan. The MSB capacitors $\left(\mathrm{C}_{9}-\mathrm{C}_{5}\right)$ follow a common-centroid configuration to minimize the errors from the non-uniform oxide growth in the MIM capacitors. However, the smaller LSB capacitors $\left(\mathrm{C}_{4}-\mathrm{C}_{0}\right)$ have been placed close to the bottom-plate switches to simplify the routing, thereby reducing the parasitic capacitance and resistance of the interconnection. Post-layout simulations showed that the reduced parasitic of the employed partial common-centroid layout results in better DAC linearity, compared to a capacitor array with a full common-centroid layout (where the LSB capacitors were placed in the middle of the array). Based on the simulations, the DAC with the partial common-centroid layout had a peak DNL of +0.18/-0.20 LSB and INL of $+0.30 /-0.23 \mathrm{LSB}$, while the DAC with a full common-centroid layout had a peak DNL of $+0.35 /-0.16 \mathrm{LSB}$ and INL of $+0.40 /-0.36 \mathrm{LSB}$.

\subsubsection{Switch Design}

The top-plate sampling switch was implemented using transmission gate, shown in Fig. 4.6, to achieve full-range input sampling. The switch together with the DAC capacitor array acts as the sample-and-hold circuit of the ADC. In Sec. 2.1.4, Eq. (2.10) derives the minimum track bandwidth of a sampling circuit. In this design, the sampling time is determined by the system clock, which is $\mathrm{N}+2$ times the sampling rate. Hence, we have

$$
f_{3 d B}>\frac{(N+1) \cdot(N+2) \cdot \ln 2}{\pi} f_{S} .
$$

Based on Eq. (4.1), for a 10-bit 1-kS/s SAR ADC, the required minimum $f_{3 d B}$ is about $30 \mathrm{kHz}$. Taking account of the 14-pF sampling capacitance, the switch on-resistance $\left(R_{O N}\right)$ should be designed to be less than $380 \mathrm{k} \Omega$.

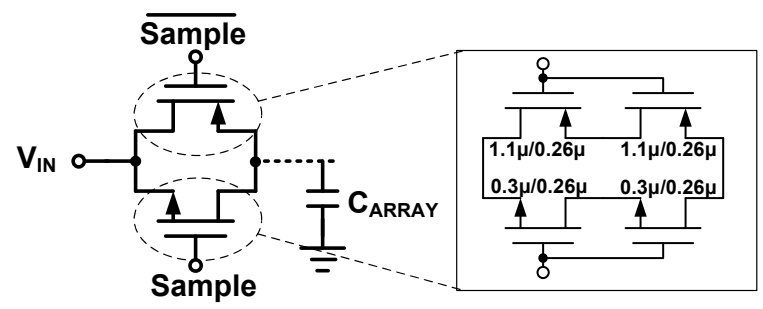

Figure 4.6: Top-plate sampling switch.

Apart from the bandwidth requirement, the voltage droop introduced by the leakage current of the switch can also degrade the sampling accuracy due to the long conversion time. The sub-threshold leakage current of the transistor is the dominant leakage contributor to the switch. In addition, the leakage current shows nonlinear 
dependence on the input-output voltage difference across the switch, thus introducing harmonic distortion. Increasing the channel length is an effective solution to reduce the sub-threshold leakage current. To further reduce the leakage current, we have utilized a two-transistor stack [48] (shown in Fig. 4.6). Figure 4.7 shows the simulated sub-threshold leakage currents of two different switches versus their input voltages. The figure compares the leakage current of a single transistor with a channel length of $4 L_{\min }$ to two transistors in series with channel lengths of $2 L_{\min }$. It can be seen that the stacked transistors show lower leakage for small input voltages in the range from $0 \mathrm{~V}$ to $0.1 \mathrm{~V}$.

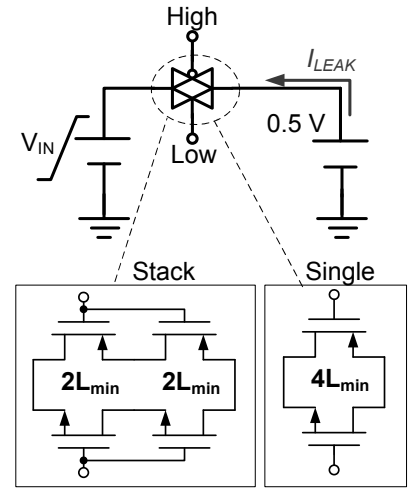

(a)

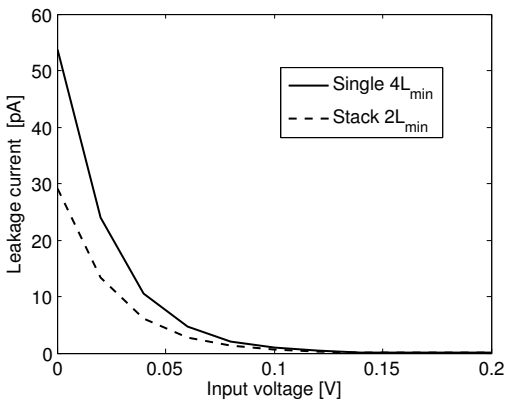

(b)

Figure 4.7: Simulated leakage current of the sampling switch: (a) test-bench (b) leakage current versus input voltage.

To determine the channel length of the stacked transistors, the sampling circuit was simulated at $1-\mathrm{kHz}$ sampling frequency with full-range input signal for three different switch lengths $\left(L_{m i n}+L_{m i n}, 2 L_{m i n}+2 L_{m i n}\right.$, and $\left.3 L_{m i n}+3 L_{m i n}\right)$. The frequency of the input signal was swept from near-DC to near-Nyquist bandwidth. The voltage of the sampled output signal was recorded at the end of the hold phase to track the voltage droop. The simulated worst-case SNDR of the recorded voltage was found at near-Nyquist operation. The resulted SNDR for three different switch lengths were $60.9 \mathrm{~dB}, 67.4 \mathrm{~dB}$, and $67.5 \mathrm{~dB}$, respectively. Thus increasing the total channel length beyond $4 L_{\min }\left(2 L_{\min }+2 L_{\min }\right)$ did not introduce much benefit for the leakage reduction. Hence, in this work, the channel length of the stacked transistors was chosen to be $2 L_{\min }(0.26 \mu \mathrm{m})$.

Furthermore, we sized the transistor width and simulated the switch $R_{O N}$ over the entire input range under $-40^{\circ} \mathrm{C}$ at the slow process corner. The simulated maximum $R_{O N}$ based on the chosen transistor widths $(0.3 \mu \mathrm{m}$ for NMOS and $1.1 \mu \mathrm{m}$ for PMOS) is about $80 \mathrm{k} \Omega$. With the total array capacitance of $14 \mathrm{pF}$, the $f_{3 d B}$ of the 
sampling circuit is then calculated to be about $140 \mathrm{kHz}$, which gives a design margin with more than four times the required minimum $f_{3 d B}$.

At the bottom-plate sides, inverters connect the capacitors to the power rails. Ideally, minimum-size transistors can be used for all the inverters because of the low sampling rate, thus minimizing power consumption. In practice, however, special care must be taken during sizing of the MSB inverter. The NMOS top-plate sampling switch introduces parasitic PN-junction on the top-plate node. After a near-ground input voltage is sampled, during the MSB to MSB-1 transition, the voltage on the top-plate node can undershoot below ground, forward-biasing the $\mathrm{PN}$-junction and causing charge loss. To avoid the undershoot voltage, the PMOS transistor in the MSB inverter was sized up to six times the minimum width.

\subsubsection{Dynamic Latch Comparator}

The dynamic latch comparator [49] is shown in Fig. 4.8. Buffers have been used to make the output loading identical. A succeeding SR latch stores the comparison result for the entire clock cycle.

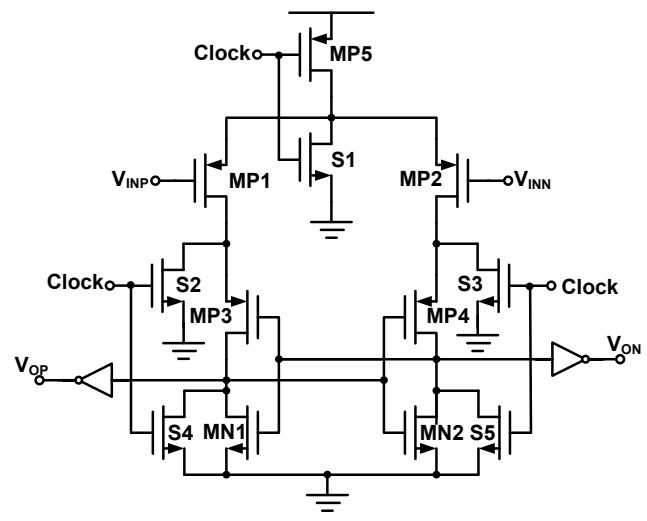

Figure 4.8: Dynamic latch comparator [49].

Since the input common-mode voltage of the comparator is kept at mid-rail voltage, the total comparator offset appears as static offset, which does not affect the linearity of the ADC [47]. Though the offset of the comparator does not affect the accuracy, it will decrease the input voltage range, thus degrading the signal-to-noise ratio. Monte Carlo simulations of the comparator offset [50] showed a total offset voltage of $35.1 \mathrm{mV}$ with $3-\sigma$ being considered. The simulated offset voltage decreases the SNR by $0.3 \mathrm{~dB}$, thereby introducing an ENOB loss of 0.05 bit. 


\subsubsection{SAR Control Logic}

For low power SAR control logic, we investigated both synchronous and asynchronous solutions. Asynchronous processing [51] has been frequently used for high-speed SAR ADCs in order to avoid a high-frequency system clock. The SAR control logic starts the conversion on the rising edge of a sampling clock, and triggers the internal comparison from MSB to LSB successively. The delay, usually generated by an inverter line [46], has a large dependency on process, voltage, and temperature variations, which makes it difficult to ensure the DAC settling. Moreover, the shortcircuit current caused by the slow transition of the inverters introduce extra power consumption [46].

The proposed ADC utilizes a synchronous SAR logic, shown in Fig. 4.9. It generates the sample signal and the switch control signals for the DAC. The operation of its multiple-input 10-bit shift resister is similar to [52]. A 4-bit counter and a decoder generate the control signals for the 10-bit shift register. The entire logic uses 16 transmission-gate DFFs and the decoder has been optimized for minimum logic depth and gate count. Fig. 4.10 shows the time sequence of the SAR logic. A 12-kHz system clock has been used, and the sampling clock of $1 \mathrm{kHz}$ is generated by the SAR logic.

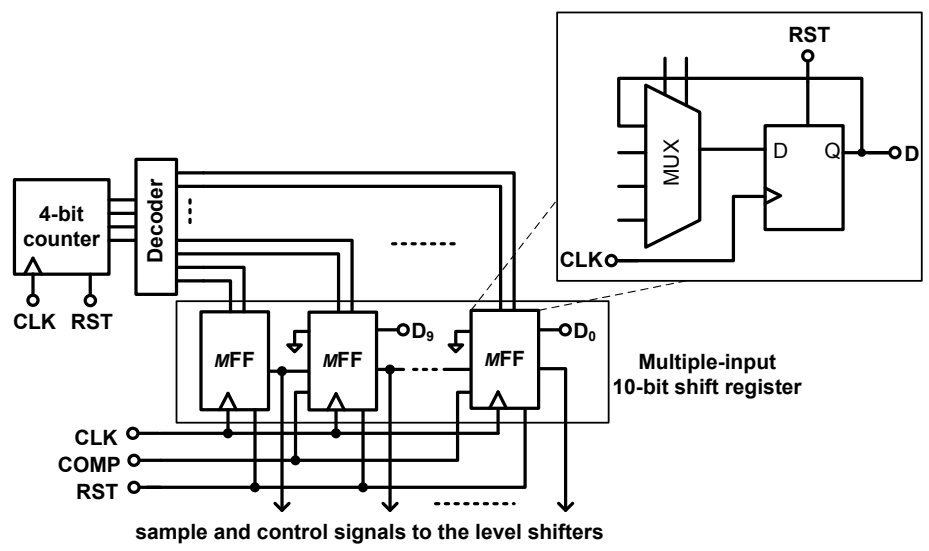

Figure 4.9: SAR control logic.

Since the operating frequency of the SAR logic is $12 \mathrm{kHz}$, and its switching activity is not high, the leakage power dominates the total power consumption. Several techniques have been used to reduce the leakage currents, including increased channel length, minimum transistor width, and replacing the gate transistors with stacked pairs [53].

To further reduce the switching and leakage power consumption, a dual-supply voltage scheme has been employed, allowing the SAR logic to operate at $0.4 \mathrm{~V}$. A 


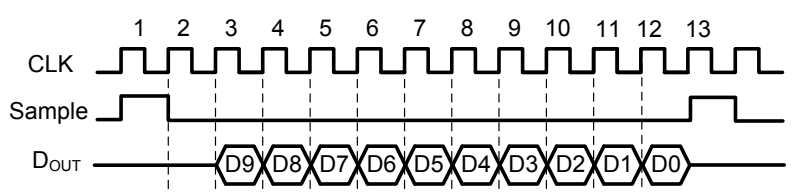

Figure 4.10: Time sequence of the synchronous SAR control logic.

conventional level shifter, shown in Fig. 4.11, has been used to convert the logic levels between the SAR and the analog parts. In the entire ADC, twelve level shifters have been used: ten for the DAC control signals, one for the sampling signal of the top-plate switch, and one for the clock signal of the comparator.

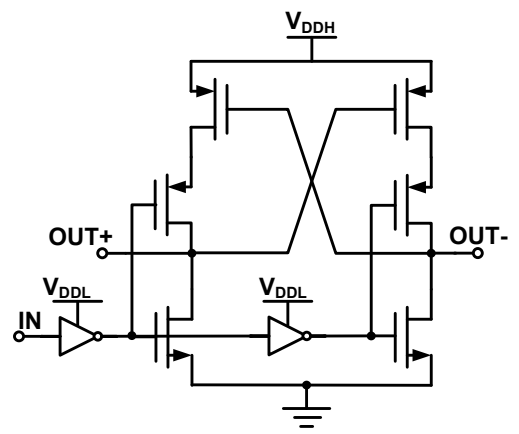

Figure 4.11: A conventional level shifter.

\subsubsection{Measurement Results}

The prototype SAR ADC with a core area of $357 \times 536 \mu \mathrm{m}^{2}$ was designed and fabricated in a general purpose $0.13-\mu \mathrm{m}$ one-poly six-metal (1P6M) CMOS process. It was packaged in a $1.27 \mathrm{~mm}$ pitch J-Leaded Chip Carrier (JLCC) package. A photograph of the chip is shown in Fig. 4.12. The unmarked part around the ADC core includes the decoupling capacitors and the I/O buffers for the pads.

Histogram test [54] was conducted to measure the linearity of the ADC. A fullswing, differential sinusoidal input near DC frequency with amplitude of $1 \mathrm{~V}$ was applied to the 1-kS/s ADC. Fig. 4.13 shows the measured DNL and INL error with respect to the output code. The peak DNL error is $+0.54 /-0.61 \mathrm{LSB}$, and the peak INL error is $+0.45 /-0.46$ LSB.

The SNDR of the ADC was measured using tone testing. A fast Fourier transform (FFT) of the 1-kS/s ADC at near-Nyquist operation is shown in Fig. 4.14(a). The amplitude of the test stimulus was set to -0.5 dBFS. The measured SNDR is $56.7 \mathrm{~dB}$, 


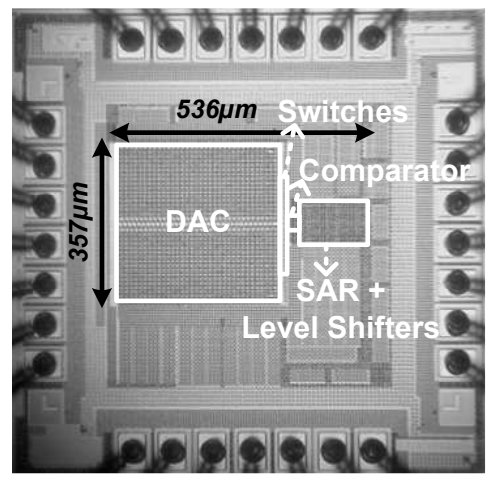

Figure 4.12: Die photograph of the ADC in $0.13-\mu \mathrm{m}$ CMOS technology.
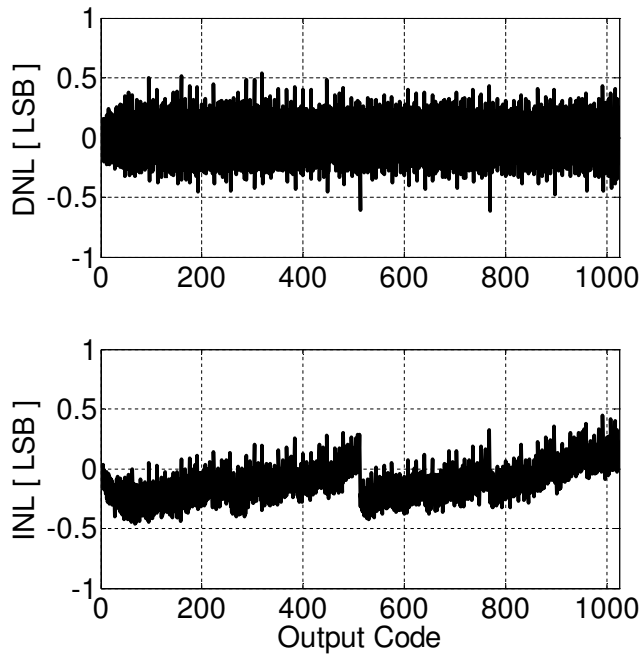

Figure 4.13: Measured DNL and INL errors. 
providing 9.1 ENOB. Fig. 4.14(b) shows the ENOB of this ADC with respect to the input frequency, where the ENOB remains almost constant over the entire bandwidth. Hence, the effective resolution bandwidth (ERBW) is higher than the Nyquist bandwidth.

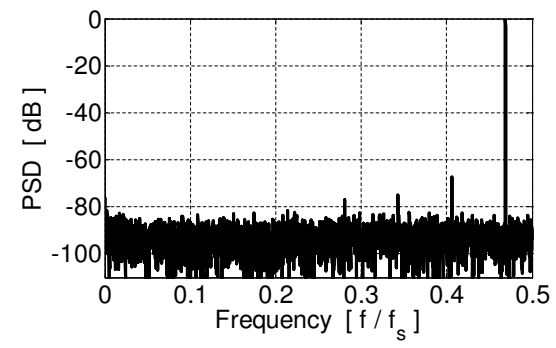

(a)

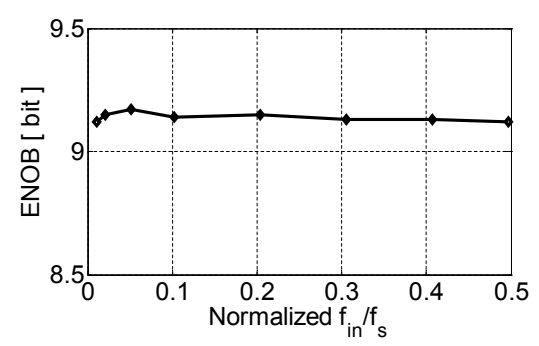

(b)

Figure 4.14: Dynamic performance: (a) Measured 8,192-point FFT spectrum at $1 \mathrm{kS} / \mathrm{s}$ (b) ENOB versus input frequency.

Multiple supply voltage domains were utilized, allowing detailed measurement of the power consumption in the DAC, comparator, and SAR control logic, respectively. The total measured power consumption of the 1-kS/s ADC is $53 \mathrm{nW}$ in dual-supply mode ( $V_{D D H}$ of $1.0 \mathrm{~V}$ for DAC and comparator, and $V_{D D L}$ of $0.4 \mathrm{~V}$ for SAR logic) and $72 \mathrm{nW}$ in 1-V single-supply mode. The measured leakage power of the digital part is $13 \mathrm{nW}$ in dual-supply mode and $22 \mathrm{nW}$ in $1-\mathrm{V}$ single-supply mode, which constitutes $25 \%$ and $31 \%$ of the total power consumption, respectively. It implies that the digital leakage power consumption is a major contributor to the total power consumption.

The power of the level shifters was simulated based on post-layout extraction. In dual-supply mode, the level shifters consume $12 \mathrm{nW}$; in 1-V single-supply mode, they consume $10 \mathrm{nW}$. Excluding the 10-nW power consumption of level shifters from the total $72 \mathrm{nW}$ would result in a total ADC power consumption of $62 \mathrm{nW}$ in the single-supply mode. This indicates that the voltage scaling has reduced the overall power consumption of the ADC by $15 \%$ without any loss in performance. Fig. 4.15 shows the power breakdown of the ADC in the two modes. Table 4.1 summarizes the measured performance of the ADC.

The power and dynamic performance of the 1-kS/s ADC under different supply settings were measured. The figure-of-merit (FOM) which has been used to compare the ADC performance is defined as

$$
F O M=\frac{\text { Power }}{\min \left\{f_{S}, 2 \times E R B W\right\} \times 2^{E N O B}} .
$$

Table 4.2 shows the measurement results under different supply settings. The 


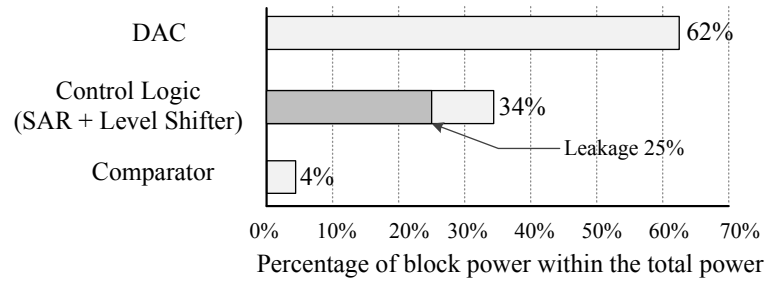

(a) Dual-supply mode $V_{D D H}=1.0 \mathrm{~V}$ and $V_{D D L}=0.4 \mathrm{~V}$

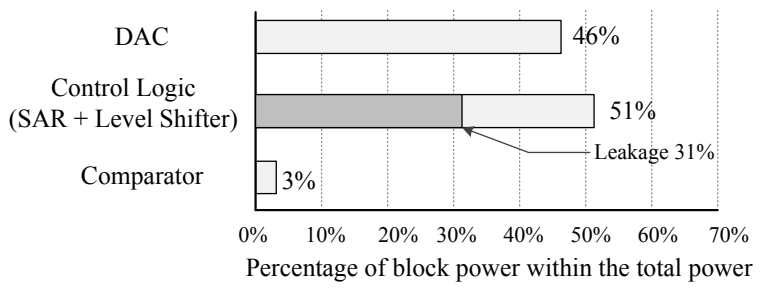

(b) Single-supply mode $V_{D D H}=V_{D D L}=1.0 \mathrm{~V}$

Figure 4.15: The ADC power breakdown in dual and single supply modes, where the percentage of digital leakage power is indicated by dark color.

Table 4.1: ADC Measurement Summary

\begin{tabular}{|c|c|c|}
\hline \multicolumn{3}{|c|}{ ADC Performance } \\
\hline Technology & \multicolumn{2}{|c|}{ standard $0.13-\mu \mathrm{m} \mathrm{CMOS}$} \\
\hline Core area $\left[\mathrm{mm}^{2}\right]$ & \multicolumn{2}{|c|}{0.19} \\
\hline Resolution [bits] & \multicolumn{2}{|r|}{10} \\
\hline Input range & \multicolumn{2}{|r|}{$0-V_{D D}$} \\
\hline Sampling rate $[\mathrm{kS} / \mathrm{s}]$ & \multicolumn{2}{|r|}{1} \\
\hline DNL [LSB] & \multicolumn{2}{|r|}{$+0.54 /-0.61$} \\
\hline INL [LSB] & \multicolumn{2}{|r|}{$+0.45 /-0.46$} \\
\hline SNDR (at Nyquist) [dB] & \multicolumn{2}{|r|}{56.7} \\
\hline SFDR (at Nyquist) [dB] & \multicolumn{2}{|r|}{67.6} \\
\hline \multicolumn{3}{|c|}{ ADC Power Breakdown } \\
\hline Supply voltage [V] & & \\
\hline Comparator, DAC & 1.0 & 1.0 \\
\hline SAR & 0.4 & 1.0 \\
\hline Comparator $[\mathrm{nW}]$ & 2 & 2 \\
\hline Capacitive DAC [nW] & 33 & 33 \\
\hline Control Logic (SAR+Level Shifter) [nW] & 18 & 37 \\
\hline Total Power $[\mathrm{nW}]$ & 53 & 72 \\
\hline
\end{tabular}


ADC achieves the optimal performance at $V_{D D H}=1.0 \mathrm{~V}$ and $V_{D D L}=0.4 \mathrm{~V}$ with the lowest FOM of $94.5 \mathrm{fJ} / \mathrm{C}$ onversion. The ADC is compared with other works in Table 4.7.

Table 4.2: ADC Performance Under Different Supply Settings

\begin{tabular}{|l|c|c|c|c|}
\hline$V_{D D H} / V_{D D L}[V]$ & $0.8 / 0.4$ & $1.0 / 0.4$ & $1.0 / 1.0$ & $1.2 / 1.2$ \\
\hline Power [nW] & 45 & 53 & 72 & 94 \\
\hline ENOB (at Nyquist) [bit] & 8.7 & 9.1 & 9.1 & 9.2 \\
\hline FOM [fJ/Conv.] & 108.1 & 94.5 & 129.4 & 159.8 \\
\hline
\end{tabular}

\subsection{A 3-nW 9.1-ENOB SAR ADC in 65nm CMOS Process}

\subsubsection{Comparison in Leakage between $0.13 \mu \mathrm{m}$ and $65 \mathrm{~nm}$ CMOS Processes}
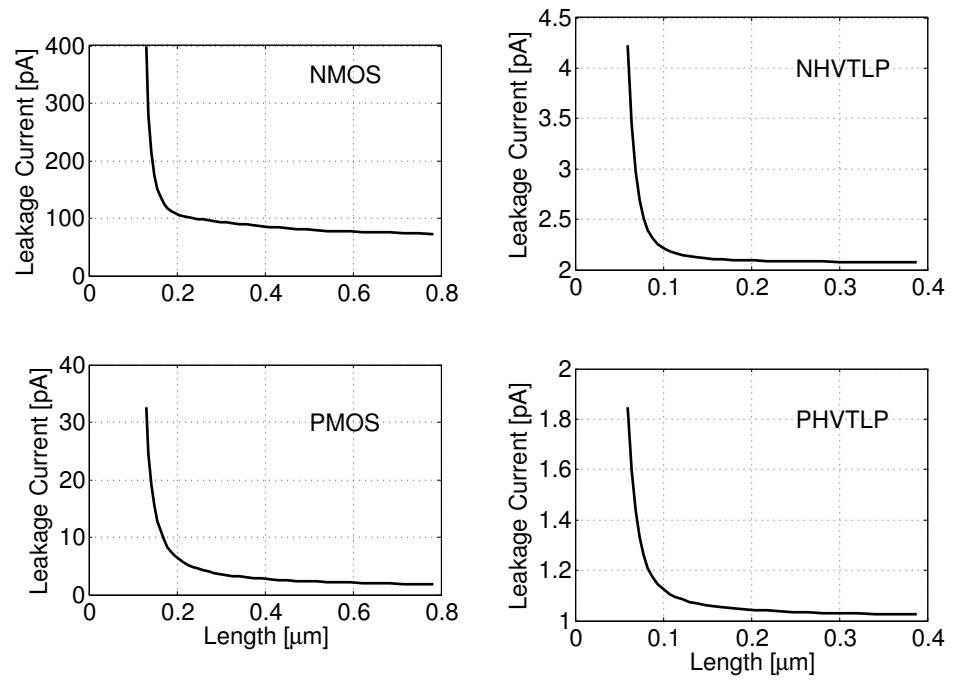

(a)

(b)

Figure 4.16: Simulated transistor sub-threshold leakage current versus channel length with minimum width at $1-\mathrm{V}$ supply, typical corner, and $27^{\circ} \mathrm{C}$ : (a) in standard $0.13 \mu \mathrm{m}$ CMOS process; (b) in low-power $65 \mathrm{~nm}$ CMOS process. 
The previous design in $0.13 \mu \mathrm{m}$ CMOS process has shown that leakage is critical to ADC performance. Fig. 4.16 compares the transistor sub-threshold leakage current between the standard $0.13 \mu \mathrm{m}$ CMOS process and the low-power $65 \mathrm{~nm}$ CMOS process. It can be seen that the leakage current is substantially reduced in $65 \mathrm{~nm}$ CMOS process due to the availability of high- $\mathrm{V}_{\mathrm{T}}$ transistors.

\subsubsection{ADC Architecture}

The architecture of the proposed SAR ADC is shown in Fig. 4.17. It comprises a differential capacitive DAC, a dynamic latch comparator, and a synchronous SAR control logic.

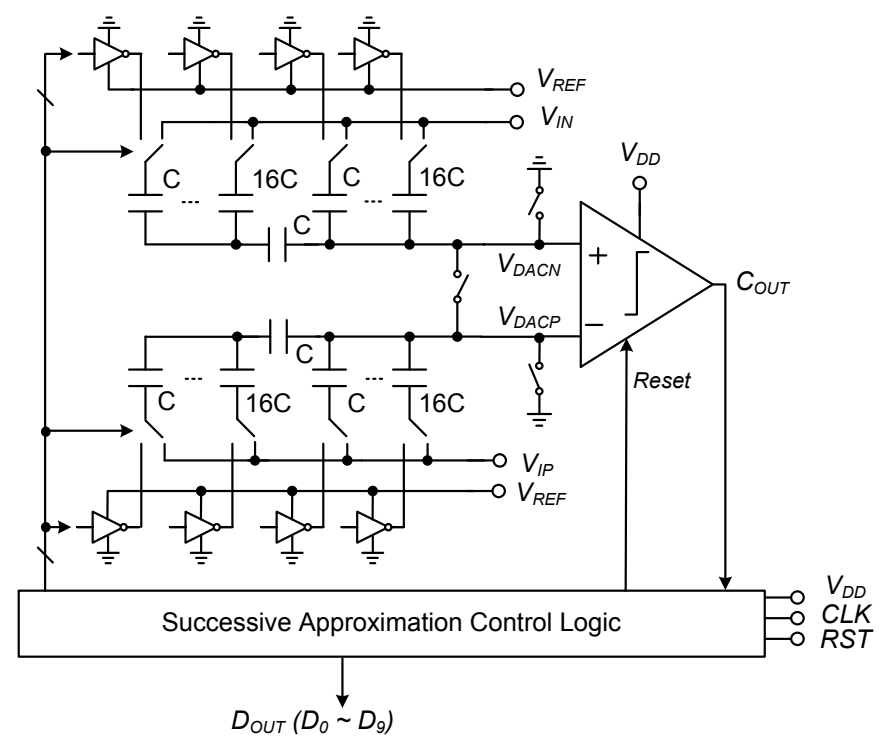

Figure 4.17: SAR ADC architecture.

The metal capacitor has a matching of $0.5 \% \mu \mathrm{m}$ and a density of $0.4 \mathrm{fF} / \mu \mathrm{m}^{2}$. For a 10-bit binary-weighted capacitive array, it leads to a minimum unit capacitance of $0.2 \mathrm{fF}$ according to Eq. (2.20). While the minimum metal capacitance defined by the design-kit is $10 \mathrm{fF}$, which is much larger than necessary to meet the linearity requirements, thereby resulting in considerably large array capacitors and high switching power consumption. Hence, we implemented a split binary-weighted capacitive array with 5-bit sub-DAC and 5-bit main-DAC. According to Eq. (2.34), it requires a larger unit capacitor of $6 \mathrm{fF}$. Further considering the design margin, a 15-fF unit capacitance is chosen, which arrives at a total array capacitance of $945 \mathrm{fF}$. 
Since the array capacitance is significantly reduced, the charge injection during sampling will degrade the conversion accuracy, and therefore bottom-plate sampling technique is used to minimize the error. When a full-range input voltage is sampled on the bottom-plates, the top-plate nodes are usually reset to the input common-mode. Otherwise, the DAC outputs will go beyond the rails for certain input range. In this work, we use a sampling approach to avoid introducing the extra reset voltage source, and at the same time the approach still ensures full-range input sampling without degrading the signal-to-noise ratio.

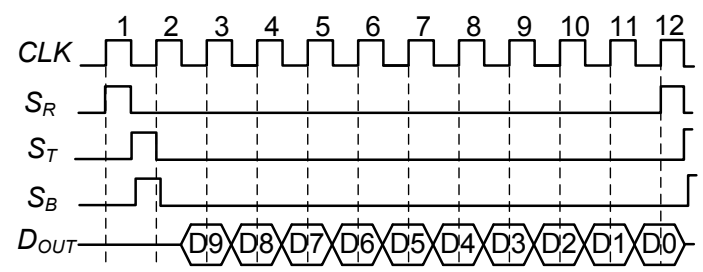

Figure 4.18: Time sequence of SAR ADC.

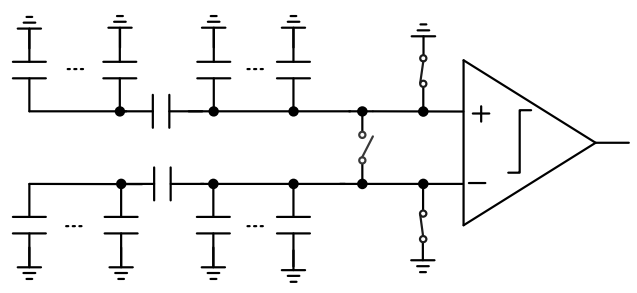

(a)

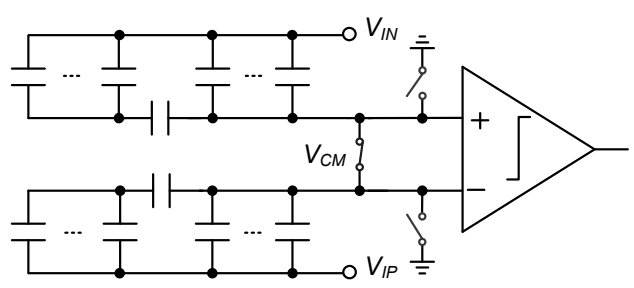

(b)

Figure 4.19: DAC arrays during (a) reset phase (b) sampling phase.

Figure 4.18 illustrates the time sequence of the SAR ADC, which takes 11 clock cycles in total to complete one conversion. The first clock cycle is divided into reset phase and sampling phase. Fig. 4.19 depicts the switching status of DAC arrays during 
the two phases. In reset phase (Fig. 4.19(a)), the control signal, indicated as $S_{R}$ in Fig. 4.18, becomes high. The capacitors are discharged to ground. When it comes to the sampling phase (Fig. 4.19(b)), the control signals, driving the bottom-plate switches and the top-plate connection switch, turn to high, which are indicated as $S_{T}$ and $S_{B}$ in Fig. 4.18, respectively. The bottom-plates of the differential capacitor arrays are then connected to the differential input. The top-plates are shorted, and their voltage becomes the input common-mode. Consequently, only the differential-mode input is sampled on the array, which makes the DAC outputs always remain within the rails. To minimize the charge injection error, the top-plate connection switch is turned off before the bottom-plate switches. As shown in Fig. 4.18, the signal $S_{B}$ is delayed by a series of weak inverters compared to the signal $S_{T}$.

In our previous work, a dual-supply voltage scheme was utilized. The additional supply and level shifters increase the design complexity and consume extra power. In this work we chose a single supply for simplicity. In addition, a combination of high- $\mathrm{V}_{\mathrm{T}}$ and standard $-\mathrm{V}_{\mathrm{T}}$ transistors is utilized to minimize the leakage while still ensuring sufficient speed at low voltages (down to $0.6 \mathrm{~V}$ ), thereby achieving the optimal energy efficiency. Our measurement results (in Sec. 4.2.8) show that the ADC achieves $4.5 \mathrm{fJ} /$ conversion-step with $8.8 \mathrm{ENOB}$ at $0.6 \mathrm{~V}$ and $1 \mathrm{kS} / \mathrm{s}$.

\subsubsection{Capacitive DAC}

As aforementioned, the DAC with split-array, shown in Fig. 4.17, is composed of two binary-weighted capacitive arrays with an attenuation capacitor in the middle. The attenuation capacitor is set to be the unit capacitance instead of a fractional value for ease of layout and good matching. Removing a dummy unit capacitor at the end of the sub-DAC introduces gain error about 1 LSB. If necessary, the gain error can be calibrated in the digital domain.

A careful layout to avoid linearity degradation is important as well. Fig. 4.20 illustrates the DAC layout floor plan for one differential branch. Both the mainDAC $\left(\mathrm{C}_{9}-\mathrm{C}_{5}\right)$ and sub-DAC $\left(\mathrm{C}_{4}-\mathrm{C}_{0}\right)$ use a partial common-centroid strategy. The MSB capacitors follow a common-centroid configuration to minimize the errors from the non-uniform oxide growth in the metal capacitors. The smaller LSB capacitors are placed close to the switch network to simplify the interconnection.

The parasitic capacitance associated with the bridge capacitor degrades the ADC performance. Fig. 4.21 shows two connection cases of the parasitic capacitor. Intuitively, the capacitor plate which contributes the major parasitic capacitance should be connected to the main-DAC, which is 'Case 1' in Fig. 4.21. Since the sub-DAC interpolates between transition voltages generated by the main-DAC, if the parasitic capacitor is connected to the sub-DAC, it will introduce more step variations to the transfer curve. To verify the effect by simulation, the capacitive DAC was implemented under three cases: 1 ) parasitic capacitance of $10 \mathrm{fF}$ connected to the main-DAC; 2) parasitic capacitance of $10 \mathrm{fF}$ connected to the sub-DAC; and 3) ideal bridge capacitor 


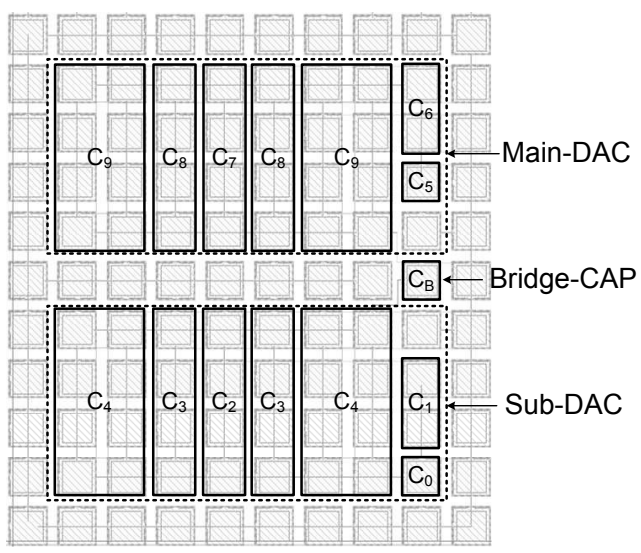

Figure 4.20: Layout floor plan of the capacitor array. The capacitors, not indicated in the figure, are dummies.

without parasitics. The ADC dynamic performance at near-DC input frequency of the three cases based on transistor-level simulations are given in Table 4.3. Compared to the ideal case, the parasitic capacitor degrades the ADC performance. Moreover, the worst case happens when the parasitic capacitor is connected to the sub-DAC. Therefore, in the DAC layout, the plate of the bridge capacitor which contributes more parasitic capacitance is deliberately connected to the main-DAC.

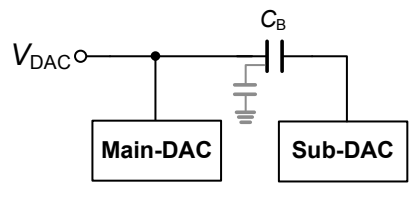

Case 1

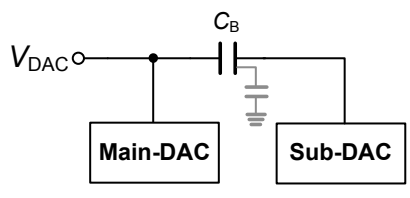

Case 2

Figure 4.21: The parasitic capacitance of the bridge capacitor: 1) connected to the mainDAC (Case 1); 2) connected to the sub-DAC (Case 2).

\subsubsection{Switch Design}

The switches of the capacitor array are shown in Fig. 4.22. Multi- $\mathrm{V}_{\mathrm{T}}$ design approach has been used. Three types of transistors are provided by the process: high- $\mathrm{V}_{\mathrm{T}}$, standard- $\mathrm{V}_{\mathrm{T}}$, and low- $\mathrm{V}_{\mathrm{T}}$. Because low- $\mathrm{V}_{\mathrm{T}}$ transistors have high leakage current, they are not suitable for this design. Hence, only high- $\mathrm{V}_{\mathrm{T}}$ and standard- $\mathrm{V}_{\mathrm{T}}$ transistors are used, and they are indicated by HVT and SVT in the figure, respectively. 
Table 4.3: ADC Dynamic Performance With Respect to Bridge Capacitor Connection

\begin{tabular}{|l|c|c|c|c|}
\hline & SNR & SNDR & THD & Unit \\
\hline Case 1 (to Main-DAC) & 60.77 & 60.39 & -71.13 & $\mathrm{~dB}$ \\
\hline Case 2 (to Sub-DAC) & 60.24 & 59.77 & -69.64 & $\mathrm{~dB}$ \\
\hline Case 3 (Ideal) & 60.84 & 60.45 & -71.14 & $\mathrm{~dB}$ \\
\hline
\end{tabular}

Since the conversion time is long and the top-plate nodes are floating during the conversion, to minimize the voltage droop caused by leakage current, the top-plate switches are all using high- $\mathrm{V}_{\mathrm{T}}$ NMOS. Moreover, in order to further reduce the leakage current, the reset-switches utilize a two-transistor stack and long channel length $(1 \mu \mathrm{m})$, and the connection-switch uses four times the minimum channel length.

At the bottom-plates of the capacitive array, transmission gates with standard$\mathrm{V}_{\mathrm{T}}$ devices are used to speed up the DAC settling when $V_{I N}$ is near mid-range. However, the inverters, switching the bottom-plate nodes between ground and $V_{R E F}$, are implemented with high- $\mathrm{V}_{\mathrm{T}}$ transistors to reduce the leakage current from $V_{R E F}$.

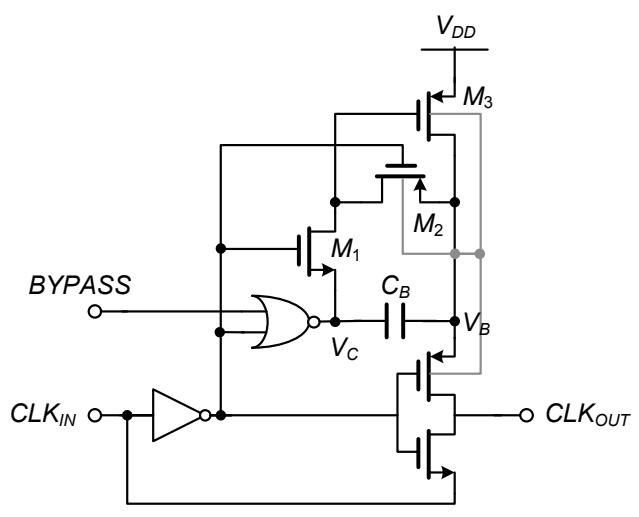

Figure 4.23: Voltage boosting circuit with bypass function.

Though the top-plate connection-switch with high- $\mathrm{V}_{\mathrm{T}}$ devices effectively reduces the sub-threshold leakage current, its high on-resistance will introduce severe distortions at low supplies when it passes midrail voltages. In view of this, a voltage boosting circuit, similar to [55], is used to drive the connection-switch at low supply voltages. As depicted in Fig. 4.23, when the input clock goes low, $\mathrm{M}_{1}$ and $\mathrm{M}_{3}$ turn on, $\mathrm{M}_{2}$ turns off, $V_{C}$ goes to ground, $V_{B}$ connects to $V_{D D}$, and the output clock goes low; when the input clock goes high, $\mathrm{M}_{1}$ and $\mathrm{M}_{3}$ turn off, $\mathrm{M}_{2}$ turns on, $V_{C}$ connects to $V_{D D}, V_{B}$ is then boosted to $2 V_{D D}$, and the output clock goes high with $2 V_{D D}$. In practice, due to charge sharing between $\mathrm{C}_{\mathrm{B}}$ and the parasitic capacitance associated to node $V_{B}$, the boosted output is less than $2 V_{D D}$. 


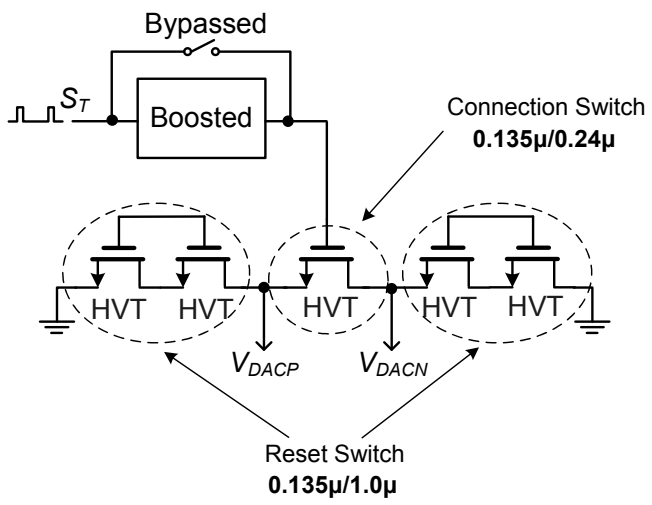

(a)

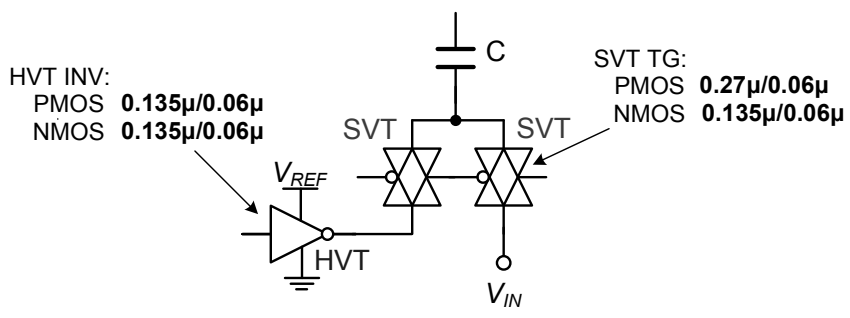

(b)

Figure 4.22: (a) Top-plate switches. (b) Bottom-plate switches.

The boosting circuit can be disabled by setting the BYPASS signal to high. The output of the NOR gate is then kept at ground, and the output logic high always equal to $V_{D D}$.

\subsubsection{Dynamic Latch Comparator}

The dynamic latch comparator [56] is shown in Fig. 4.24. It has no static biasing. Buffers are connected successively to make the output loading identical. A followed cross-coupled NAND gates store the comparison result for an entire clock cycle. The reset signal is synchronous with the clock of the SAR control logic.

Similarly, the comparator utilizes a combination of high- $\mathrm{V}_{\mathrm{T}}$ and standard- $\mathrm{V}_{\mathrm{T}}$ transistors to minimize the leakage while ensuring the target speed at low voltages. Standard- $\mathrm{V}_{\mathrm{T}}$ devices are used for the differential input transistors and the crosscoupled inverters to enhance the comparison speed. A high- $\mathrm{V}_{\mathrm{T}}$ PMOS is implemented for the bias transistor to reduce the leakage current from the supply. 


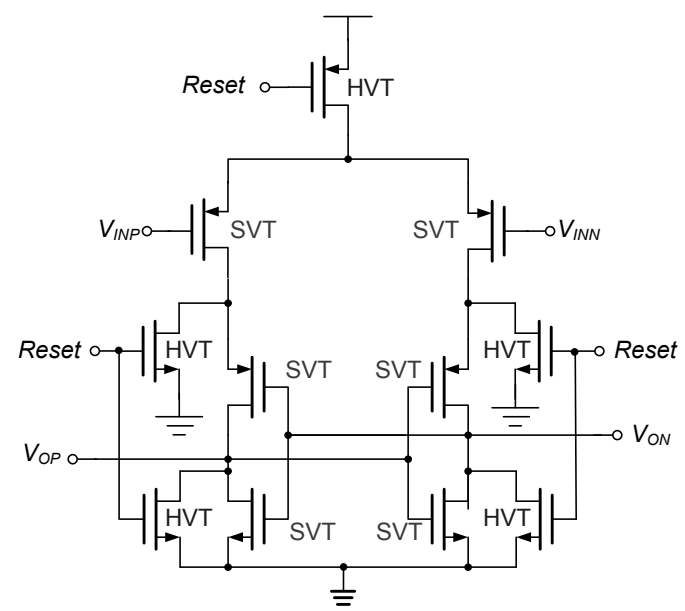

Figure 4.24: Dynamic latch comparator with high- and standard- $\mathrm{V}_{\mathrm{T}}$ transistors.

Since the input common-mode voltage of the comparator is kept at mid-rail voltage, the comparator offset appears as static offset, which does not affect the ADC linearity [47]. However, the offset decreases the input voltage range, thus degrading the signal-to-noise ratio. Monte Carlo simulations of the comparator offset showed a standard deviation of $17 \mathrm{mV}$. Assuming $3 \sigma$ is considered, it results in a total offset voltage of $51 \mathrm{mV}$, decreasing the SNR by $0.7 \mathrm{~dB}$. The decreased SNR introduces an ENOB loss of 0.1 bit.

\subsubsection{SAR Control Logic}

The SAR control logic is shown in Fig. 4.25. It consists of a shift register, a set of bit latches, a sample signal generator, and a combinational switching logic. Due to the low sampling rate, static logic instead of dynamic one is used for the register and latches to avoid charge leakage. On the other hand, since speed is not a major concern, the logic circuits are designed with minimum size.

A latch instead of a DFF is used to store the comparator decision, thus reducing the transistor count and lowering the power consumption. However, special care must be taken during generating the latch signal. The latch may miss the current comparator result and record the successive one due to an improper latch signal. In this design, the output signal from the corresponding-order DFF of the shift register, indicated in Fig. 4.25, is used to clock the latch. 


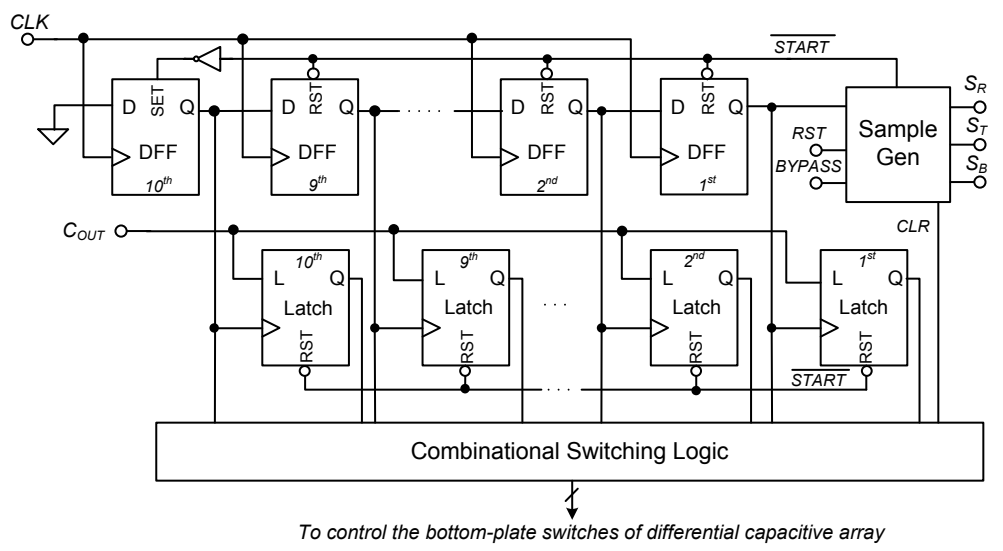

Figure 4.25: SAR digital control logic.

\subsubsection{Chip Implementation}

A micrograph of the entire ADC in 65nm CMOS process is shown in Fig. 4.26. The core occupies $0.037 \mathrm{~mm}^{2}$, while the remaining area in the figure is used for supply decoupling. For clarity, the right part of Fig. 4.26 shows the ADC layout including the differential capacitive DAC, the comparator, and the SAR digital logic.

Table 4.4: Simulated Power Consumption

\begin{tabular}{|c|c|c|}
\hline & Schematic [nW] & Post-layout [nW] \\
\hline Comparator & $0.09(6 \%)$ & $0.25(9 \%)$ \\
\hline DAC & $0.75(48 \%)$ & $1.10(39 \%)$ \\
\hline SAR logic & $0.73(46 \%)$ & $1.46(52 \%)$ \\
\hline Total & $1.57(100 \%)$ & $2.81(100 \%)$ \\
\hline
\end{tabular}

Table 4.4 shows the power breakdown of the ADC at $0.7 \mathrm{~V}$ and $1 \mathrm{kS} / \mathrm{s}$ according to simulations. By subtracting the schematic-based total power consumption from the post-layout-based one, the power consumption of parasitics is approximated to be $1.24 \mathrm{nW}$, which constitutes a large portion of the total power (44\%). This can be improved by optimizing the layout, but it also demenstrates that the interconnection becomes more significant for such a low-speed design with small transistor dimensions in deep-submicron technology. In addition, according to the schematic-level simulation, the total leakage power is $0.67 \mathrm{nW}$, which contributes $43 \%$ of the total power consumption. 


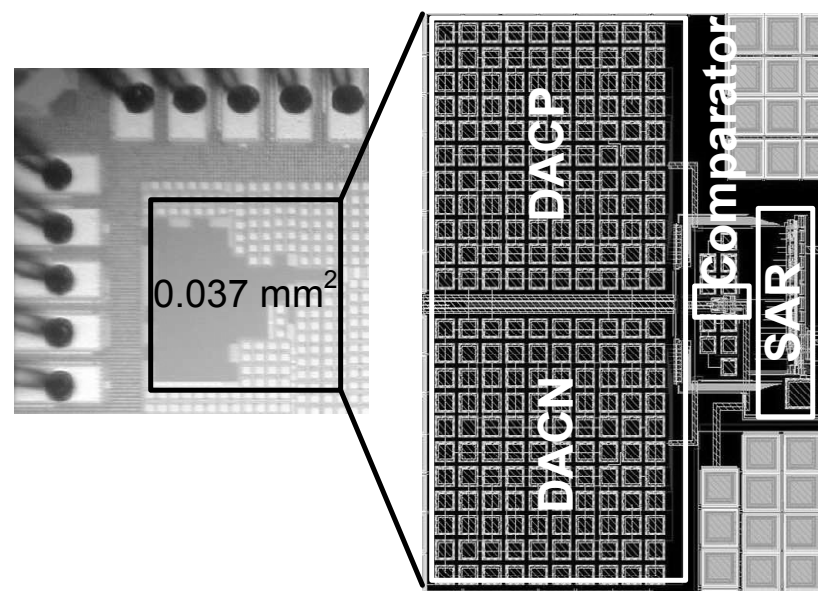

Figure 4.26: Die photo and layout view of the ADC in $65 \mathrm{~nm}$ CMOS process.

\subsubsection{Measurement Results}

Histogram and tone test were conducted to measure the static and dynamic performance of the $1-\mathrm{kS} / \mathrm{s}$ ADC, respectively. At $V_{D D}$ of $0.7 \mathrm{~V}$, the measured peak DNL error is $+0.48 /-0.55 \mathrm{LSB}$, and the peak INL error is +0.52/-0.61 LSB, as shown in Fig. 4.27.

At the same supply, the measured FFT spectrums of the ADC at both near-DC and near-Nyquist operations are depicted in Fig. 4.28. The amplitude of the test stimulus was set to $-0.65 \mathrm{dBFS}$. At near-DC operation, the measured SNDR is $57.1 \mathrm{~dB}$, providing 9.2 ENOB. When the input frequency increases to near-Nyquist bandwidth, the measured SNDR becomes $56.6 \mathrm{~dB}$, resulting in 9.1 ENOB.

The measured total power of the $1-\mathrm{kS} / \mathrm{s}$ ADC at $0.7 \mathrm{~V}$ is $3 \mathrm{nW}$, leading to a FOM of $5.5 \mathrm{fJ} /$ conversion-step. Table 4.5 summarizes the measured performance of the ADC.

The power and ENOB of the 1-kS/s ADC under different supply voltages were also measured, and the results are shown in Table 4.6. At $V_{D D}$ from $0.6 \mathrm{~V}$ to $1.0 \mathrm{~V}$, the sampling switch is boosted; At $V_{D D}$ of $1.2 \mathrm{~V}$, the boosted circuit is bypassed. The ADC achieves the minimum FOM of $4.5 \mathrm{fJ} /$ conversion-step at $0.6 \mathrm{~V}$. Table 4.7 compares the measurement results of this work and previous work to published SAR ADCs with comparable sampling rates.

The predicted mismatch-limited energy bounds together with Nyquist SAR ADC survery data from [7] and results of our implememted ADCs are shown in Fig. 4.29. The parameters in the power model are kept the same except for the supply voltage which is scaled to $0.5 \mathrm{~V}$ for a lower bounds. We note that the 53-nW ADC in $0.13 \mu \mathrm{m}$ 

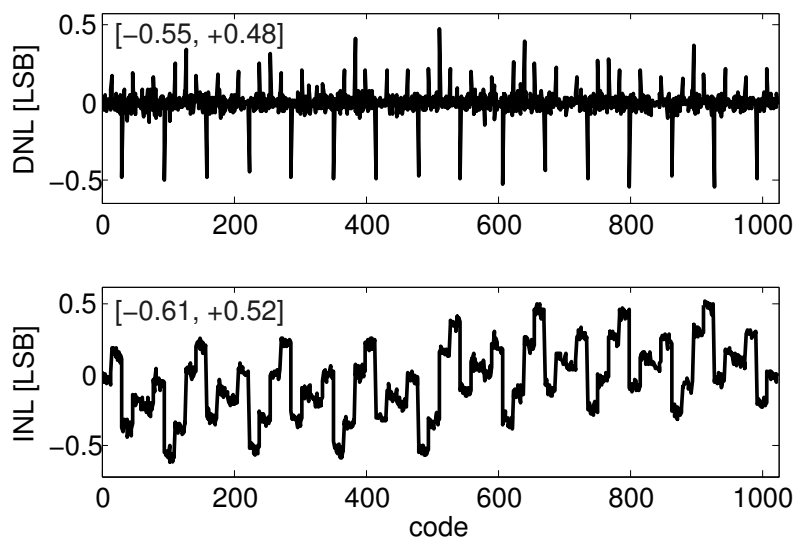

Figure 4.27: Measured DNL and INL errors of 1-kS/s 0.7-V ADC.

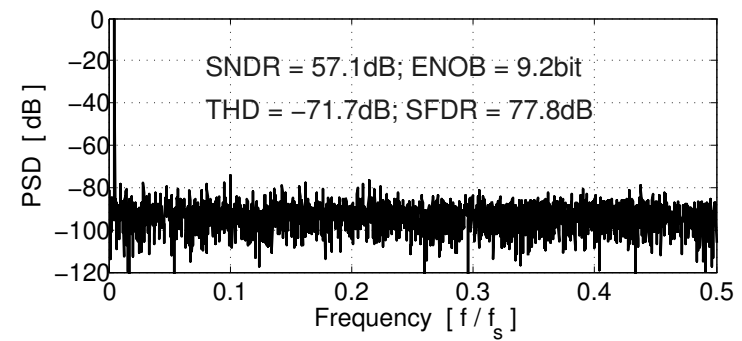

(a)

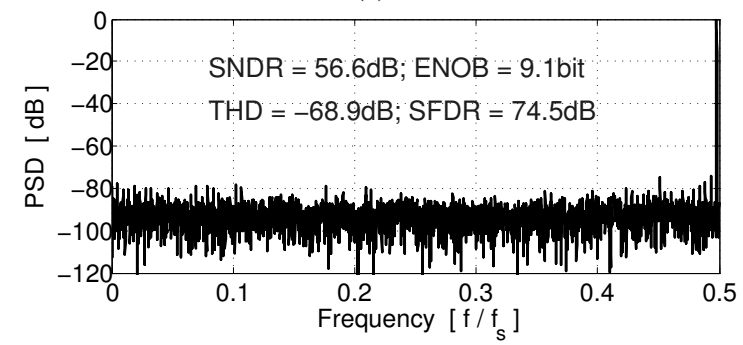

(b)

Figure 4.28: Measured 8,192-point FFT spectrums of 1-kS/s 0.7-V ADC: (a) near DC (b) near Nyquist. 
Table 4.5: ADC Measurement Summary

\begin{tabular}{|l|c|}
\hline Technology & 65-nm CMOS \\
\hline Core area $\left[\mathrm{mm}^{2}\right]$ & 0.037 \\
\hline Resolution [bit] & 10 \\
\hline Input range [V] & $0-V_{D D}$ \\
\hline Sampling rate [kS/s] & 1 \\
\hline Supply voltage [V] & 0.7 \\
\hline DNL [LSB] & $+0.48 /-0.55$ \\
\hline INL [LSB] & $+0.52 /-0.61$ \\
\hline SNDR (near Nyquist) [dB] & 56.6 \\
\hline SFDR (near Nyquist) [dB] & 74.5 \\
\hline THD (near Nyquist) [dB] & -68.9 \\
\hline ENOB [bit] & 9.1 \\
\hline Total power [nW] & 3 \\
\hline FOM [fJ/Conv.] & 5.5 \\
\hline
\end{tabular}

Table 4.6: Measured ADC Performance Under Different Supply Voltages

\begin{tabular}{|c|c|c|c|}
\hline$V_{D D}[\mathrm{~V}]$ & Power [nW] & ENOB [bits] & FOM [fJ/Conv.] \\
\hline 0.6 & 2 & 8.8 & 4.5 \\
\hline $\mathbf{0 . 7}$ & $\mathbf{3}$ & $\mathbf{9 . 1}$ & $\mathbf{5 . 5}$ \\
\hline 0.8 & 4 & 9.1 & 7.3 \\
\hline 0.9 & 5 & 9.2 & 8.5 \\
\hline 1.0 & 6 & 9.2 & 10.2 \\
\hline $1.2^{\dagger}$ & 8 & 9.0 & 15.6 \\
\hline
\end{tabular}

${ }^{\dagger}$ The sampling switch is not boosted.

Table 4.7: ADC Comparison

\begin{tabular}{|l|c|c|c|c|c|c|c|}
\hline & {$[57]$} & {$[58]$} & {$[11]$} & {$[12]$} & {$[13]$} & Previous work & This work \\
\hline Technology [nm] & 350 & 180 & 180 & 65 & 65 & 130 & 65 \\
\hline Sampling rate [kS/s] & 1 & 4.1 & 1 & 20 & 40 & 1 & 1 \\
\hline Area [mm²] & N/A & 0.11 & 0.05 & 0.212 & 0.076 & 0.19 & 0.037 \\
\hline Supply voltage [V] & 1.0 & 0.5 & 0.5 & 0.55 & 0.6 & $1.0 / 0.4$ & 0.7 \\
\hline Power [nW] & 230 & 850 & 2.5 & 206 & 97 & 53 & 3 \\
\hline ENOB [bit] & 10.2 & 6.9 & 8.52 & 8.84 & 10.1 & 9.1 & 9.1 \\
\hline FOM [fJ/Conv.] & 195 & 1700 & 6.8 & 22.3 & 2.2 & 94.5 & 5.5 \\
\hline
\end{tabular}


CMOS is a bit far from the bounds because leakage power consumption, which is neglected in our power analysis for simplicity, does take a significant portion of the total power, thereby degrading the energy efficiency. Compared to the 53-nW ADC, the 3-nW ADC in $65 \mathrm{~nm}$ CMOS is closer to the bounds owing to the well-optimized design in a low-leakage advanced technology.

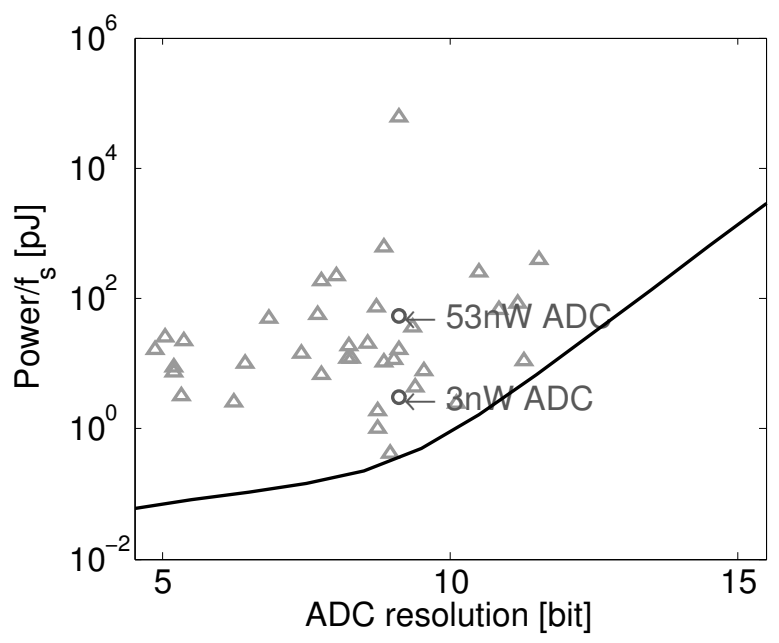

Figure 4.29: Predicted mismatch-limited SAR ADC energy bounds (solid line) together with Nyquist SAR ADC survey data $(\Delta)$ and the two implemented ADCs (o). 


\section{Chapter 5}

\section{Digital Calibration of Capacitive DAC}

In Chapter 3, the power cost of performing mismatch compensation by simply sizing up the capacitor has been analyzed. In order to relax the mismatch requirement on the capacitor sizing while still ensuring enough linearity for high-resolution SAR ADCs, in this chapter, a digital calibration technique is proposed and the design overhead introduced by the calibration is estimated.

\subsection{Introduction}

One of the limiting factors for SAR ADCs to achieve high-resolution is the capacitor mismatch. To minimize the mismatch error, one way is to size up the capacitor by paying the penalty for higher power, larger area, and lower speed. An alternative approach is to using a smaller capacitive array together with some form of calibration. Analog calibrations using additional digital-to-analog converters (DACs) were proposed in the 80 s $[59,60]$. Recently, more efforts $[38,39,61]$ have been made to push calibration into the digital domain since the scaling of CMOS technology offers advantages of digital circuitry in terms of speed, power, and integration.

Equalization is a common digital calibration technique. Split-ADC equalization [38] implements two identical ADCs on the same chip, captures the difference between their outputs, and simultaneously calibrates their bit weights until the captured difference is minimized. To simplify this technique, a self-equalization approach [39] works on a single ADC by employing an offset double conversion scheme. The efficiency of equalization heavily depends on the input signal to achieve and accelerate convergence. Different from equalization, in this chapter, we introduce a digital calibration technique without applying any external input signal. It utilizes 
a concept similar to that of [61], in which the lower bit weights are assumed to be correct and the higher bit weights are measured and expressed by the lower bit weights. The concept applies to the non-binary-weighted converters. Compared to a binary-weighted one, the non-binary-weighted converter introduces redundancy during bit decision with more conversion steps, as shown in Fig. 5.1. Owing to the redundancy, the approximation of higher weights by lower weights is still feasible even when certain amount of capacitor variation is taken into account. In [61], the converter combines a binary-weighted network with a ladder structure. To deal with the comparator offset during the calibration, the converter needs to do double conversion with a specific requirement on the time sequence, which increases design complexity. Different from [8], in this work, the converter employs a sub-radix-2 single-array capacitive DAC, which has a better tolerance to parasitics. Instead of double-conversion, single normal successive approximation is performed. The effect of comparator offset during calibration is then canceled by a subtraction in the digital post-processing phase. Since the entire procedure does not affect the original conversion sequence of a charge-redistribution SAR ADC, the calibration circuit can be easily incorporated into the converter without modifying its main architecture and digital logic.

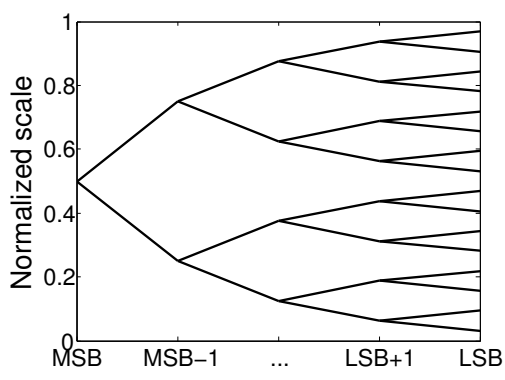

(a)

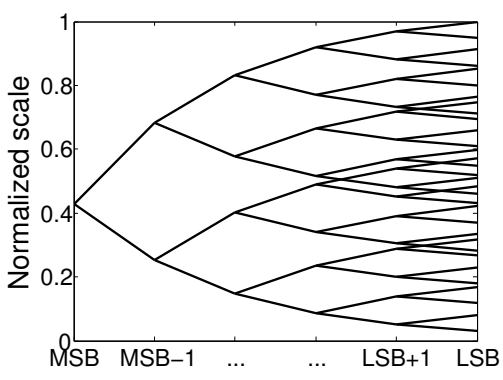

(b)

Figure 5.1: Decision tree of: (a) a binary-weighted converter; (b) a non-binary-weighted converter.

Considering the calibration applies to a sub-radix-2 single-array capacitive network, an in-depth understanding of the characteristics of such networks is beneficial. The effect of capacitor variation on the design of non-binary-weighted capacitive DAC in terms of radix, conversion steps, and accuracy is analyzed. In addition, one important property associated with the non-binary-weighted network - one-side redundancy is described. It limits the ability of the ADC in correcting early decision errors due to insufficient settling or coupled noise by later decision steps. To solve this problem, a solution briefly mentioned in [62] is introduced, and a mathematical derivation of the solution is further provided. 


\subsection{Capacitor Property}

\subsubsection{Capacitor Voltage Dependence}

The dependence of capacitance on voltage can be expressed by

$$
C=C_{0}\left(1+\alpha_{1} V+\alpha_{2} V^{2}\right),
$$

where $V$ represents the potential difference between the capacitor plates, and $\alpha_{1}$ and $\alpha_{2}$ are the linear and quadratic voltage coefficients, respectively. Depending upon the capacitor plate, the dielectric materials and the thickness, $\alpha_{1}$ can vary from 10's to 100 's $\mathrm{ppm} / \mathrm{V}$, and $\alpha_{2}$ rarely exceeds $10 \mathrm{ppm} / \mathrm{V}^{2}$ [63].

When considering the ratio of two capacitors, if the voltage difference is constant, the variations on the absolute value of the capacitor tend to cancel. However, during conversion, the array capacitors are switched to different voltages. In [63], it is analyzed that the error caused by capacitor voltage dependence under $V=5 \mathrm{~V}$ represents a 0.0004 -percent full-scale integral nonlinearity, corresponding to about $\pm 0.25 \mathrm{LSB}$ in a 16-bit ADC. Following this analysis, if a smaller voltage, e.g. $1 \mathrm{~V}$, is applied, the error is much smaller than 0.25 LSB.

\subsubsection{Capacitor Temperature Dependence}

Another important parameter for capacitors is the temperature coefficient of capacitance (TCC), given by

$$
T C C=\frac{1}{C} \frac{\mathrm{d} C}{\mathrm{~d} T} .
$$

The TCC of integrated capacitors is found to be in the range of 20 to $50 \mathrm{ppm} / \mathrm{K}$ [64]. For any power dissipation $P$, the effective temperature difference $(\Delta T)$ is calculated as

$$
\Delta T=P \times \theta,
$$

where $\theta$ is the thermal resistance in units of $\mathrm{K} / \mathrm{W}$, which is a heat property and a measurement of a temperature difference by which an object or material resists a heat flow [65]. The thermal resistance of aluminum is $0.43 \mathrm{~K} / \mathrm{W}$ [66]. Assume the power consumption of the circuitry around the capacitor is $1 \mu \mathrm{W}$, the temperature difference on the MIM capacitor is $0.43 \mu \mathrm{K}$. The relative capacitance variation with TCC of $50 \mathrm{ppm} / \mathrm{K}$ is

$$
\frac{\Delta C}{C}=T C C \times \Delta T=50 \times 10^{-6} \times 0.43 \times 10^{-6}=21.5 \times 10^{-12} .
$$

The variation is negligible. Furthermore, when considering the ratio of two capacitors, the variations on the absolute value of the capacitor tend to cancel. Therefore, temperature variations have little effect on the matching accuracy of capacitors. 
To summerize, the capacitor ratio after fabrication is quite stable. Once calibration is complete, there is no need to repeat it.

\subsection{Analysis of Non-Binary-Weighted DAC}

\subsubsection{Preliminaries}

In order to achieve $N$-bit accuracy, the non-binary-weighted converter with a radix $r(r<2)$ requires $S$ conversion steps $(S>N)$. The bit weight, which reflects the ratio between the $j$-th capacitor $C_{j}(S \geq j \geq 1)$ and the total array capacitor $C_{t o t}$ is expressed as

$$
W_{j}=\frac{C_{j}}{C_{t o t}} .
$$

Based on the numerical weights and the bit decisions $D_{i}$ generated by the converter, the normalized input voltage can be represented by

$$
V_{I N}=\sum_{i=1}^{S} D_{i} W_{i}
$$

In order to ensure enough dynamic range of the non-binary-weighted capacitive DAC with $N$-bit target accuracy, the following inequality should be satisfied [67]

$$
C_{t o t}>2^{N} C_{1}
$$

The redundancy for bit $j$ is expressed as

$$
\operatorname{Redundancy}(j)=\sum_{i=1}^{j-1} W_{i}-W_{j} .
$$

It represents the distance between the decision levels corresponding to digital code transitions involving $01 \cdots 1$ to $10 \cdots 0$. To guarantee the redundancy, it requires

$$
\sum_{i=1}^{j-1} W_{i}>W_{j}
$$

Multiplying both sides with $C_{t o t}$, Eq. (5.9) can be further expressed in terms of capacitor as

$$
\sum_{i=1}^{j-1} C_{i}>C_{j}
$$




\subsubsection{Accounting for Capacitor Variation}

In this section, we analyze the effect of capacitor variation on the two inequalities, Eq. (5.7) and Eq. (5.10).

Each array capacitor is modeled as the sum of a nominal capacitance and an error term [18]. The LSB capacitor as a unit capacitor is then modeled as

$$
C_{1}=C_{u}+\delta_{1}, \text { and } E\left[\delta_{1}^{2}\right]=\sigma_{u}^{2},
$$

where $C_{u}$ is the nominal capacitance, $\delta_{1}$ is a random variable with a zero mean and a variance of $\sigma_{u}^{2}$. Then, the rest of the capacitors in the array can be modeled as

$$
C_{j}=r^{j-1} C_{u}+\delta_{j}, \text { and } E\left[\delta_{j}^{2}\right]=r^{j-1} \sigma_{u}^{2} .
$$

We first consider Eq. (5.7) by calculating the variance of $C_{t o t}$

$$
\operatorname{Var}\left[C_{t o t}\right]=\frac{1-r^{S}}{1-r} \sigma_{u}^{2}
$$

Further considering $3 \sigma$ for high yield, Eq. (5.7) accounting for capacitor variation can be re-written as

$$
\frac{1-r^{S}}{1-r} C_{u}-3 \sqrt{\frac{1-r^{S}}{1-r}} \sigma_{u}>2^{N}\left(C_{u}+3 \sigma_{u}\right) .
$$

Note that Eq. (5.14) is a conservative bound because the correlation between the variation in $C_{1}$ and $C_{t o t}$ is ignored. With further mathematical manipulation, Eq. (5.14) is finally expressed as

$$
\frac{1-r^{S}}{1-r}-2^{N}-3\left(2^{N}+\sqrt{\frac{1-r^{S}}{1-r}}\right) \frac{\sigma_{u}}{C_{u}}>0 .
$$

In a similar manner, Eq. (5.10) accounting for capacitor variation can be written as

$$
\frac{2 r^{j-1}-r^{j}-1}{r-1}-3\left(\sqrt{r^{j-1}}+\sqrt{\frac{1-r^{j-1}}{1-r}}\right) \frac{\sigma_{u}}{C_{u}}>0 .
$$

The radix determines the bit position at which redundancy starts. No redundancy is provided for the lower-weighted bits. Given the standard deviation of the unit capacitor, Eq. (5.15) determines the minimum conversion steps under certain radix for $N$-bit accuracy, and Eq. (5.16) determines the maximum radix for a specific bit position where redundancy starts. A smaller radix introduces redundancy to more bits but also increases conversion steps. Taking a 15-bit ADC as an example and assume a unit-capacitor standard deviation of $1 \%$, the required minimum $S$ in terms of selected $r$ is given in Table 5.1, and the maximum $r$ in terms of $j$ is given in 
Table 5.2. It indicates that bit position of 4 or 5 is a reasonable starting point to generate redundancy. Fig. 5.2 depicts the tradeoffs among $\sigma_{u} / C_{u}, r$, and $S$ for 15-bit accuracy. First, the radix $r$ is calculated according to Eq. (5.16) where the redundancy starts from bit 5. Secondly, the conversion step is calculated according to Eq. (5.15) with the chosen radix. The figure shows that a larger capacitor variation requires a smaller radix and thus needs more conversion steps.

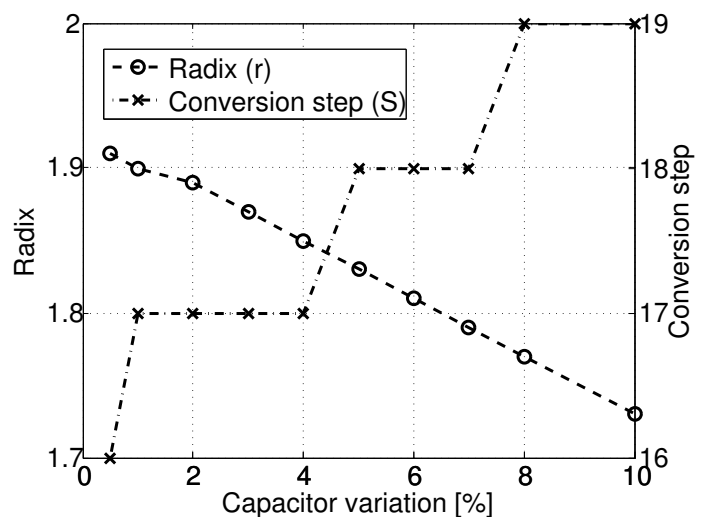

Figure 5.2: Tradeoffs among $\sigma_{u} / C_{u}, r$, and $S$ for 15-bit accuracy.

Table 5.1: Required Minimum $S$ in Terms of $r$ for $N=15$

\begin{tabular}{|c|c|c|c|c|c|c|}
\hline$r$ & 1.95 & 1.9 & 1.86 & 1.82 & 1.8 & 1.75 \\
\hline$S$ [bit] (Rounded) & 16 & 17 & 17 & 18 & 18 & 19 \\
\hline
\end{tabular}

Table 5.2: Maximum $r$ Can be Used in Terms of $j$

\begin{tabular}{|l|c|c|c|c|c|c|}
\hline$j$ & 3 & 4 & 5 & 6 & 7 & 8 \\
\hline$r$ & 1.58 & 1.82 & 1.91 & 1.96 & 1.98 & 1.99 \\
\hline
\end{tabular}

\subsubsection{Secondary Bit to Tackle One-Side Redundancy}

Based on the decision tree of a non-binary-weighted ADC, Fig. 5.3 shows the decision path after a wrong decision at MSB approximation for two normalized input voltages. Three dashed lines indicate the level of the input voltage, the weight of the MSB, and sum of the rest of the bit weights, respectively. The redundancy for the MSB, according to Eq. (5.8), is also indicated in the figure.

Now assume that a normalized input of 0.46 is applied to the ADC, as shown in Fig. 5.3(a), the expected decision for MSB should be High, but a wrong decision of 


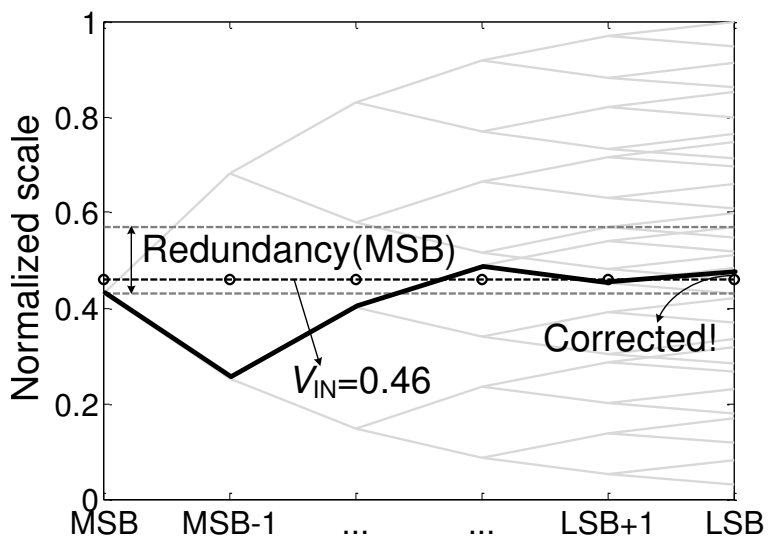

(a)

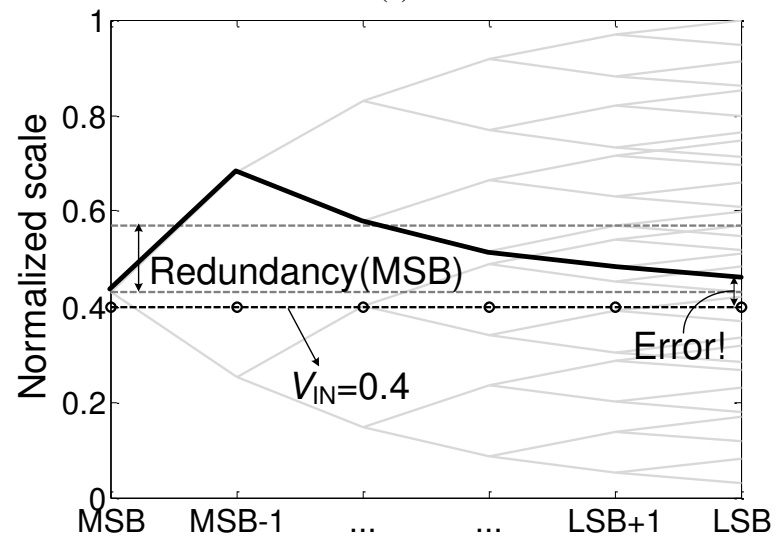

(b)

Figure 5.3: Decision path (indicated by black solid line) after a wrong decision at MSB approximation for two normalized input voltages: (a) $V_{I N}=0.46$ (b) $V_{I N}=0.4$. 
Low is made (under-approximation). In this case, as long as the input is covered by the redundancy range, the wrong decision can still be corrected by the later decision steps. Considering the opposite case of over-approximation, as shown in Fig. 5.3(b), the ADC gives a wrong decision of High for an input of 0.4 at MSB decision step. Following the decision path, this wrong decision can never be corrected by the following steps.

The redundancy only helps to correct under-approximation instead of overapproximation, because it is at one side of the decision bit. Hence, it is important to shift the comparison threshold during bit decision to the middle of the redundancy range $[39,62]$. One way is to tie an extra capacitor bank to the original array, and to increase the comparison threshold by switching on an additional capacitor together with the decision capacitor [39]. A more efficient approach is to switch on two bits simultaneously [62], in which one is the decision bit and the other is a lower-weighted bit. The secondary bit has a weight about half of the redundancy. Once the decision is made, the secondary bit goes back to its initial status. In order to locate the position of the secondary bit, we write

$$
W_{j}+\overbrace{\frac{1}{2} \cdot\left(\sum_{i=1}^{j-1} W_{i}-W_{j}\right)}^{\text {half of redundancy }}=W_{j}+W_{j-k},
$$

where $k$ indicates the distance between the secondary bit and the decision bit. With mathematical manipulation, Eq. (5.17) leads to the following approximation

$$
k \approx \frac{\log \frac{2(1-r)}{r-2}}{\log r} .
$$

Table 5.3 shows the distance between two bits in terms of radix. It can be seen that a code distance of 4 works for all the listed radix. By enabling the secondary bit during comparison, the dynamic error tolerance in terms of LSB for $N$-bit accuracy can be calculated as

$$
E_{t o l}[L S B]=\frac{\left(\sum_{i=1}^{j-1} W_{i}-W_{j}\right) / 2}{1 / 2^{N}}
$$

Table 5.3: Distance Between Two Bits in Terms of Radix

\begin{tabular}{|c|c|c|c|c|c|}
\hline radix & 1.8 & 1.82 & 1.85 & 1.86 & 1.9 \\
\hline$k$ (calculated) & 3.54 & 3.69 & 3.95 & 4.04 & 4.50 \\
\hline$k$ (rounded) & 4 & 4 & 4 & 4 & 4 or 5 \\
\hline
\end{tabular}

Taking a 15-bit 1.85-radix ADC with 17 conversion steps as an example, the ADC 
has a dynamic error tolerance of $1328,717,387, \cdots, 4,2,1 \mathrm{LSB}$ s for the first twelve conversion steps.

\subsection{Bottom-Up Weight Calibration}

Considering the variation of LSB capacitors contribute insignificant errors to the conversion accuracy, the bottom-up weight calibration approach uses the lower weights to measure and express the higher weights. The approach can be divided into two major steps: 1) the higher weights are sensed and digitized by the array via normal successive approximations; 2) the correction of the higher weights are applied digitally afterwards based on the conversion results. These two steps will be elaborated respectively in Sec. 5.4.1 and Sec. 5.4.2.

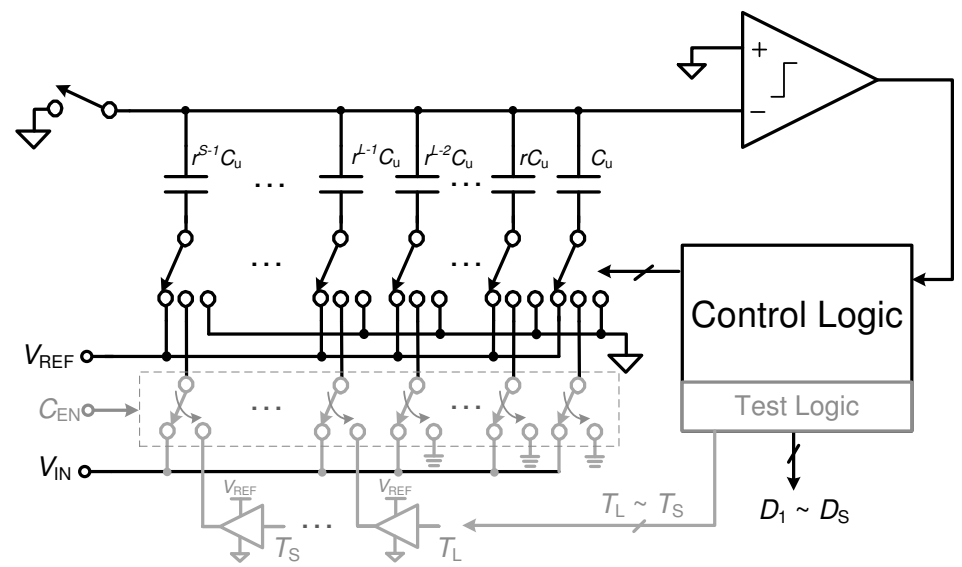

Figure 5.4: A SAR ADC incorporated weight conversion (parts indicated by grey color are the additional blocks).
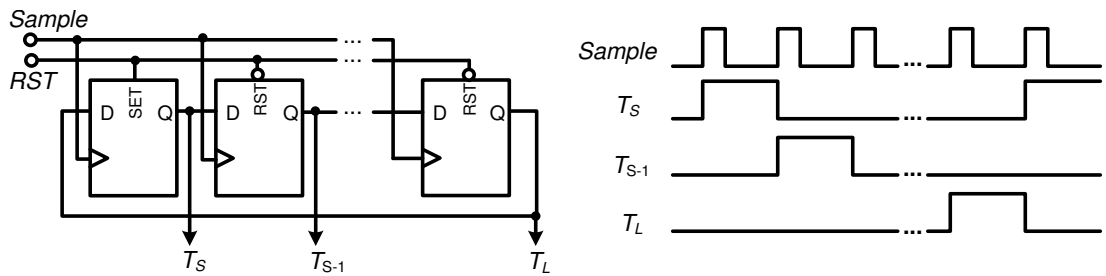

Figure 5.5: Test logic to generate the calibration signals $\left(T_{L} \sim T_{S}\right)$ and the corresponding time sequence. 


\subsubsection{Weight Conversion}

The first step of the calibration utilizes the successive-approximation algorithm, and the conversion approach can be easily integrated into a charge-redistribution SAR ADC without modifying the basic architecture, as shown in Fig. 5.4. During the calibration mode, the control signal, denoted as $C_{E N}$, switches the input branch from the input signal to the test signals. The test signals can be generated by a series of D-type flip flops, as shown in Fig. 5.5.

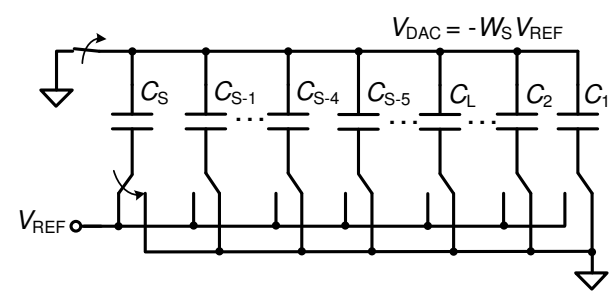

(a)

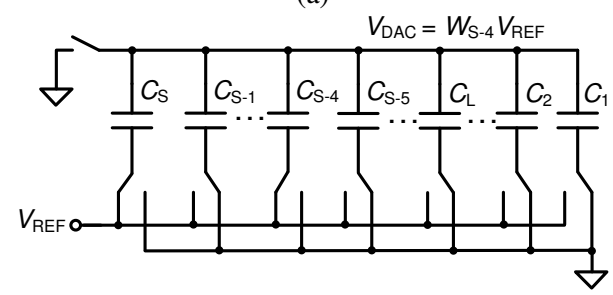

(b)

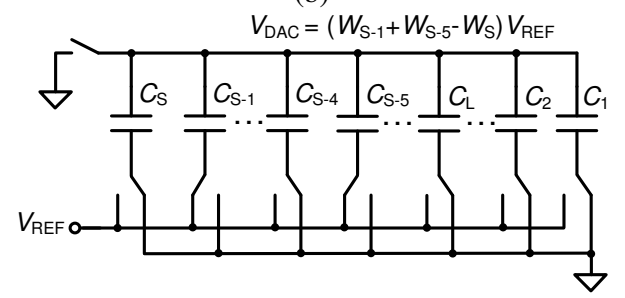

(c)

Figure 5.6: DAC switching status during conversion of MSB weight $\left(W_{S}\right)$ : (a) Sampling; (b) 1st-bit approximation; (c) 2nd-bit approximation.

The procedure of weight conversion is similar to that of a conventional SAR ADC. First, the information of the weight is sampled on the array, and then the bit decisions from MSB to LSB are successively generated. Taking the MSB weight conversion as an example, during the sampling phase, shown in Fig. 5.6(a), only the MSB capacitor $C_{S}$ is connected to the reference voltage, thereby sampling its weight information on the array. The next phase, shown in Fig. 5.6(b), is the normal approximation of 
MSB. Since the corresponding secondary bit is also switched on, it will result in a decision of Low for MSB. After the 1st-bit approximation, the MSB capacitor is connected back to the ground, while capacitors of both MSB-1 and its secondary bit are connected to the reference voltage, as shown in Fig. 5.6(c). The approximation phase continues until the LSB decision is generated. Similar conversion procedure can be applied to other higher weights until $W_{L}$.

\subsubsection{Bottom-Up Weight Calibration}

Ideally, once all the conversions of the higher weights are complete, each of these bit weights is then expressed with the sum of its lower weights, which can be written as

$$
W_{j}=\sum_{i=1}^{j-1} D_{i, j} W_{i}
$$

In reality, however, there exists offset during conversion. If the common-mode voltage of the DAC outputs is kept at a same level during the whole conversion, the effect of the signal-dependent dynamic offset from the succeeding comparator will be substantially reduced. Hence, the offset can be viewed as a constant static offset [22]. The conversion result of the bit weight, taking the offset into account, can be re-written as

$$
W_{j} \pm V_{o s}=\sum_{i=1}^{j-1} D_{i, j} W_{i}
$$

where $V_{o s}$ is the normalized offset to the reference voltage. Since the offset is static, it can be canceled by subtracting the two consecutive converted results

$$
\left(W_{j} \pm V_{o s}\right)-\left(W_{j-1} \pm V_{o s}\right)=\sum_{i=1}^{j-1} D_{i, j} W_{i}-\sum_{i=1}^{j-2} D_{i, j-1} W_{i}
$$

Finally, $W_{j}$ can be written as:

$$
W_{j}=W_{j-1}+\sum_{i=1}^{j-1} D_{i, j} W_{i}-\sum_{i=1}^{j-2} D_{i, j-1} W_{i}
$$

The calibration starts from $W_{L+1}$ by correcting its value according to Eq. (5.23). Then based on the calibrated weight, the next higher weight $W_{L+2}$ is corrected and updated. The procedure continues until all the remaining higher weights are calibrated.

The existence of offset will limit the number of weights to be converted. Fig. 5.7 shows weight conversion including either positive offset or negative offset. For the specific bit weight, its conversion range is between 0 and the sum of all its lower 
weights. Hence, in order to make it possible for the bit weight to be converted by its lower weights, the offset voltage should satisfy

$$
\begin{aligned}
& V_{o s} \leq \sum_{i=1}^{j-1} W_{i}-W_{j}, \text { for positive offset } \\
& V_{o s} \leq W_{j}, \text { for negative offset }
\end{aligned}
$$

Apart from the offset, the noise will also affect the conversion result. To overcome the noise effect, averaging can be used. The ADC first converts the higher weights for multiple runs, and then the decisions are averaged. Unlike the offset and noise, the gain error scales the bit weights by a same factor, hence it won't affect the converted weight expression. Fig. 5.8 shows the block diagram of the digital calibration logic. The power and area of the calibration logic are estimated in Sec. 5.4.3.

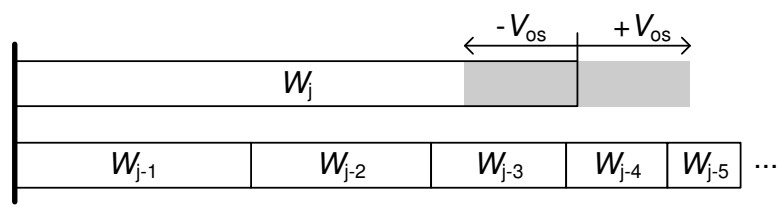

Figure 5.7: Weight conversion with the existence of offset.

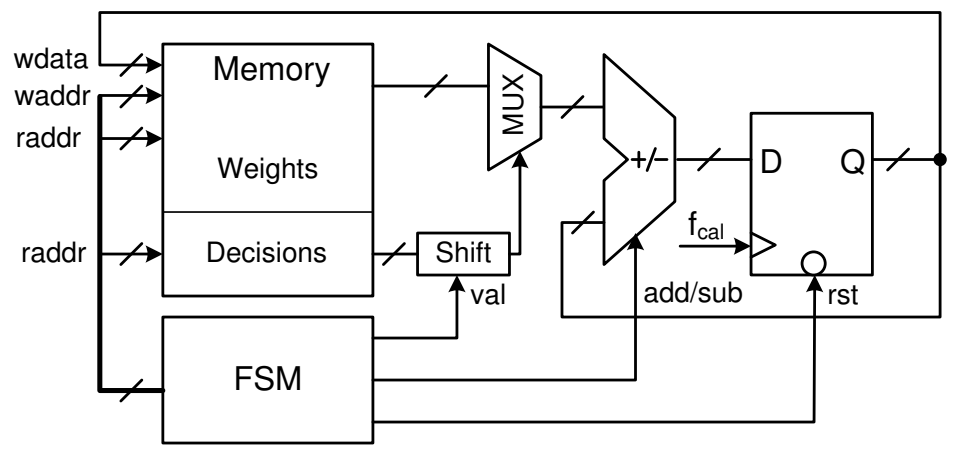

Figure 5.8: Block diagram of the digital calibration logic.

\subsubsection{Simulation Results}

A behavioral model of a 15-bit 1.85-radix 1-V SAR ADC with 17 conversion steps is created in Matlab, and the model accounts for capacitor variation, comparator offset and noise. Since we are more interested in characterizing the effectiveness of the 
calibration method, the ADC is simulated in Matlab instead of Cadence to avoid other circuit non-idealities. In addition, Matlab allows us to run extensive Monte Carlo simulations, which otherwise will be extremely time-consuming to run in Cadence.

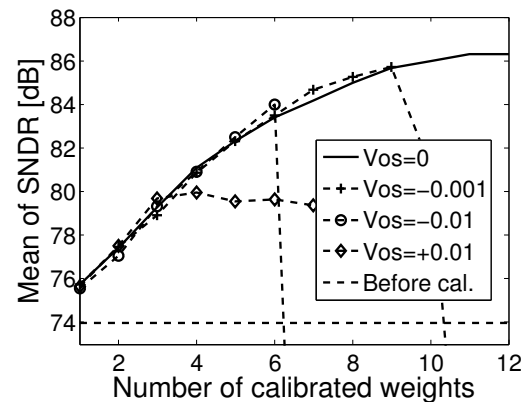

(a)

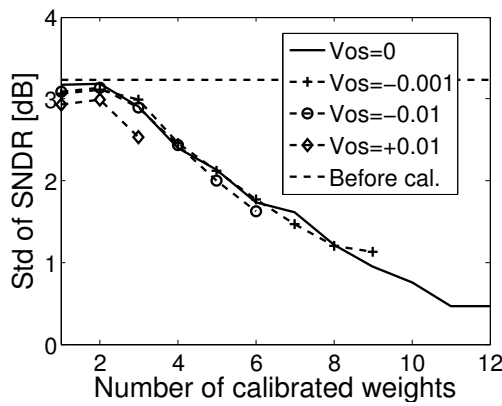

(b)

Figure 5.9: Simulated SNDR versus numbers of calibrated higher weights for $\sigma_{u} / C_{u}=3 \%$ : (a) mean (b) standard deviation.

Besides the ideal case, three more offsets are chosen: $+0.01,-0.01$, and -0.001 . The noise power is assumed to be equal to the quantization noise power of a 15-bit radix-2 ADC. The capacitor variation $\left(\sigma_{u} / C_{u}\right)$ is chosen to be $3 \%$. The ADC first calibrates the higher weights based on the average result of 16 runs. Then, it converts a sinusoidal input-signal, and the signal-to-noise-and-distortion ratio (SNDR) of the converted result is evaluated. Total 500 Monte-Carlo simulations were performed to obtain the mean and standard deviation of SNDR. Fig. 5.9 shows the simulated results versus numbers of calibrated weights. The calibration improves the performance by increasing the mean and decreasing the standard deviation. A smaller offset allows the ADC to calibrate more weights, thus leading to higher SNDR. It can also be seen that with the same amount of offset, the positive offset limits the ADC to calibrate fewer bit weights compared to the negative offset. The $+0.01-,-0.01-$, and $-0.001-V_{o s}$ respectively limit the number of calibrated weights to 3,6 , and 9 bits. When comes to the ideal case, the improvement stops at 11 bits.

Fig. 5.10(a) shows simulation results based on more values of capacitor variation. The reported SNDR is calculated as mean value subtracted by $3 \sigma$. Fig. 5.10(b) illustrates the improvement after calibration. For a sufficiently small capacitor variation, e.g. $0.1 \%$, the weights do not deviate much, and there is no need to go for calibration. For a relatively small capacitor variation, e.g. $0.5 \%$, calibrating more weights does not provide proportional improvement in SNDR. As the variation increases, the higher weights deviate more, resulting in a worse SNDR before calibration. As the number of calibrated weights increases, different matching cases almost approach the same level of performance. Furthermore, it is also informative to visualize the improvement of ADC linearity after calibration. As an example, Fig. 5.11 shows the DNL/INL plots of 


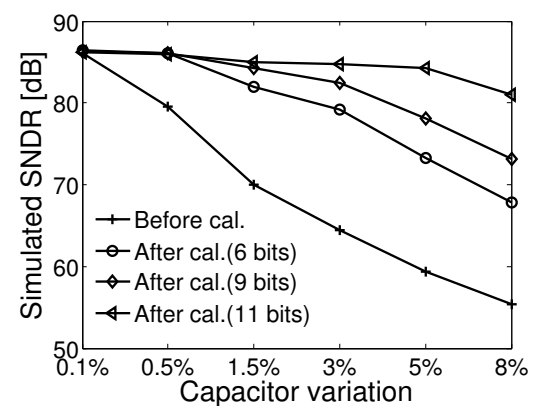

(a)

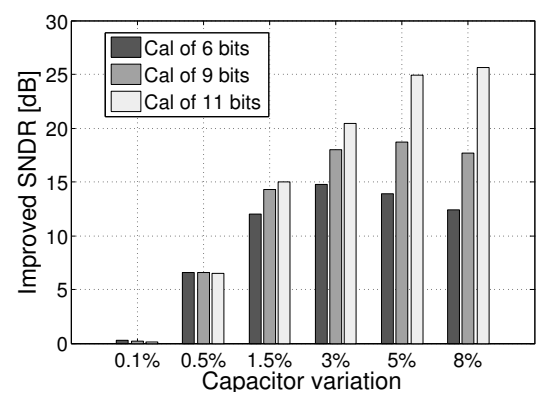

(b)

Figure 5.10: Comparison between before and after calibration: (a) simulated SNDR (mean-3 $\sigma$ ) (b) improved SNDR after calibration. (The total number of calibrated weights are indicated.)

the ADC with a capacitor variation of $1.5 \%$ and an offset of -0.001 ( 9 calibrated bits) before and after calibration. The peak DNL error is changed from $-1.00 /+0.70$ LSB to $-0.62 /+0.92 \mathrm{LSB}$, and the peak INL error from $-2.32 /+2.10 \mathrm{LSB}$ to $-0.94 /+0.76 \mathrm{LSB}$. The calibration helps to remove the missing codes and improve the ADC linearity.
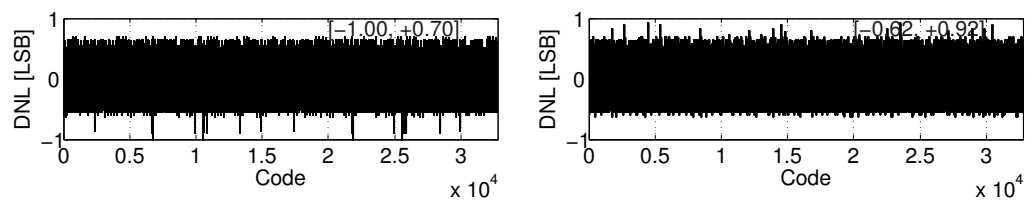

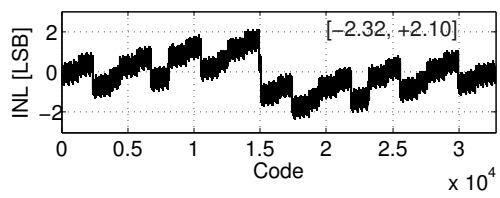

(a)

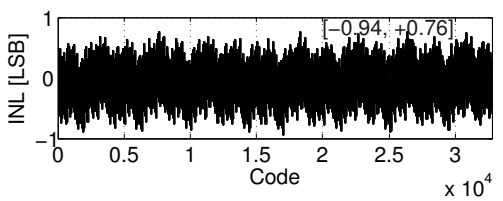

(b)

Figure 5.11: DNL/INL plots of an ADC with $\sigma_{u} / C_{u}=1.5 \%$ : (a) before calibration (b) after calibration of 9 bits.

After synthesis in Design Compiler, the estimated area of the calibration logic is about $0.01 \mathrm{~mm}^{2}$, and power about $0.05 \mathrm{~mW}$ at $10 \mathrm{MHz}$ in a standard 65-nm CMOS process. The estimated calibration time is about 500 clock cycles, in which 200 cycles for weight conversion and 300 cycles for digital calibration. Since this technique is input-independent, the digital calibration does not need to run simultaneously with the ADC. Once the weight information is sensed, digitized, and stored, the digital post-processing can be run offline at an arbitrary frequency. Table 5.4 compares this work with other digital calibration techniques. 
Table 5.4: Comparison With Other Digital Calibration Techniques

\begin{tabular}{|c|c|c|c|}
\hline & [38] & [39] & This work \\
\hline Resolution [bit] & 16 & 12 & 15 \\
\hline Radix & 2 & 1.86 & 1.85 \\
\hline Conversion steps & 16 & 14 & 17 \\
\hline Input-dependent & Yes & Yes & No \\
\hline Frequency [MHz] & 1 & 22.5 & 10 \\
\hline No. of cycles & 200,000 & 22,000 & 500 \\
\hline Area (est.) $\left[\mathrm{mm}^{2}\right]$ & 0.8 & 0.03 & 0.01 \\
\hline Power (est.) [mW] & 2.6 & 0.23 & 0.05 \\
\hline SNDR $[\mathrm{dB}]$ & - & 70 & $84^{\dagger}$ \\
\hline DNL/INL [LSB] & better than \pm 1 & - & {$[-0.62+0.92] /[-0.94+0.76]$} \\
\hline
\end{tabular}




\section{Chapter 6}

\section{A 14-Bit Redundant SAR ADC in 65nm CMOS Process}

With the development of healthcare technology, high-resolution low-speed ADCs are increasingly demanded in health monitors for capturing bioelectric signals [68]. Chapter 4 shows that SAR ADCs exhibit high energy efficiency due to their digitallyoriented nature. However, they are limited to moderate resolutions. In this chapter, a 14-bit $10-\mathrm{kS} / \mathrm{s}$ SAR ADC in $65 \mathrm{~nm}$ CMOS process is presented. To achieve the high resolution, the ADC implements a uniform-geometry non-binary-weighted capacitive DAC for linearity enhancement, employs a secondary-bit approach to dynamically shift decision levels for error correction, and uses a comparator with bias control for power reduction.

\subsection{Introduction}

The major limiting factors for SAR ADCs to achieve high resolution are capacitor mismatch and comparator noise. As discussed in Chapter 5, to minimize the capacitor mismatch, one way is to size up the capacitor by paying the penalty of higher power and larger area. An alternative approach is using a smaller capacitive array together with some form of calibration. In [38] and [39], equalization technique has been used to calibrate the bit weights via least-mean-sqaure algorithm. The efficiency of equalization heavily depends on the input signal to achieve and accelerate convergence. In [69] and [70], dithering technique has been applied to effecitvely suppress the spurs and improve the DAC linearity. The added dither is later digitally subtracted from the output code [69] or pushed out of the band using oversampling [70]. Different from equalization and dithering, a bottom-up weight calibration technique proposed in Chapter 5 is employed in this work. The technique applies to non-binary-weighted 
converters which introduce redundancy during bit decision with more conversion steps. The calibration technique assumes the lower bit weights are correct and the higher bit weights are measured and expressed by the lower bit weights.

Though regenerative latch is commonly used as the comparator for moderateresolution SAR ADCs [46, 49], the noise requirement on high-resolution comparators makes a single latch difficult to accomplish the task. Averaging the comparison result for noise-sensitive conversion steps has been utilized to enhance the accuracy of the latch, but this approach only helps the ADC to achieve SNDR up till 70dB $[69,70]$. In this work, a single-pole amplifier (SPA) with gain enhancement [63] is used as a pre-amplification stage before the dynamic latch. To decrease the static power of the SPA, the SPA is switched off once the dynamic latch completes regeneration and starts again at the beginning of the next conversion step. Moreover, considering the $\mathrm{ADC}$ is able to correct early decision errors owing to the redundancy, half of the bias current is switched off during the conversion of high-weighted bits, thereby further reducing the power consumption.

\subsection{ADC Architecture}

The architecture of the proposed SAR ADC is shown in Fig. 6.1. It comprises a non-binary-weighted differential capacitive DAC, a comparator, and a digital block including a synchronous successive approximation register and a test logic. In order to achieve 14-bit resolution, the DAC is designed to have 16 conversion steps with a radix of 1.86 .

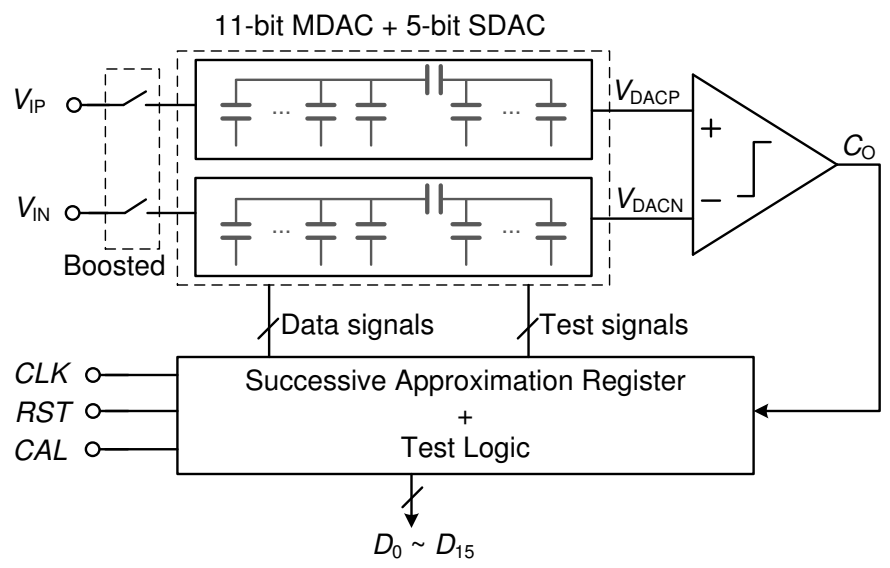

Figure 6.1: SAR ADC architecture.

While single array experiences better matching than split array, the exponentially increased number of unit capacitors for a high-resolution DAC rather introduces 
complexity during layout. Hence, a split-array with a bridge capacitor is implemented. Table 6.1 compares three different topologies of a split DAC. Among the three topologies, Topology 2 is selected in this work due to its good trade-off between matching and area. Consequently, the capacitive DAC is composed of 11-bit mainDAC (MDAC) and 5-bit sub-DAC (SDAC) with a bridge capacitor in-between.

Table 6.1: Comparison Among Three Split Topologies

\begin{tabular}{|c|c|c|c|}
\hline & Topology 1 & Topology 2 & Topology 3 \\
\hline MDAC + SDAC [bit] & $10+6$ & $11+5$ & $12+4$ \\
\hline Number of capacitors & 623 & 1096 & 2006 \\
\hline Noise-limited unit capacitance [fF] & 47 & 26 & 14 \\
\hline Estimated total area [mm $\left.{ }^{2}\right]$ & 0.107 & 0.119 & 0.132 \\
\hline
\end{tabular}

To avoid large numbers of bootstrapping switches at the bottom-plate of the capacitive array, the differential inputs are sampled on the top plate through two boosted switches. However, during calibration, the weight information is sampled through the bottom plate of the capacitors. In order to effeciently incorperate the calibration circuits into the converter, both top-plate sampling and bottom-plate sampling schemes are examinined, as shown in Fig. 6.2. Comparing the two switching procedures, the DAC status during approximation are exactly the same except for the sampling phase. In top-plate sampling scheme, the positive input is sampled on the top branch of the differential capacitive array, and the negative input on the bottom branch. While the bottom-plate sampling scheme does sampling in a opposite way. Taking this difference into account, the differential DAC array which incorperates the calibration circuit is shown in Fig. 6.3. Once the calibration mode is enabled, the top-plate switches are connected to a common-mode voltage and the bottom-plate switches are connected to the inverter bank which is controlled by the test signals. Total seven higher weights are calibrated. The gates of the inverters for lower-weighted bits are either pulled up to $V_{R E F}$ (with regard to the bottom branch) or pulled down to ground (with regard to the top branch). The calibration circuit doesn't modify the main architecture and the digital control logic of the ADC. 


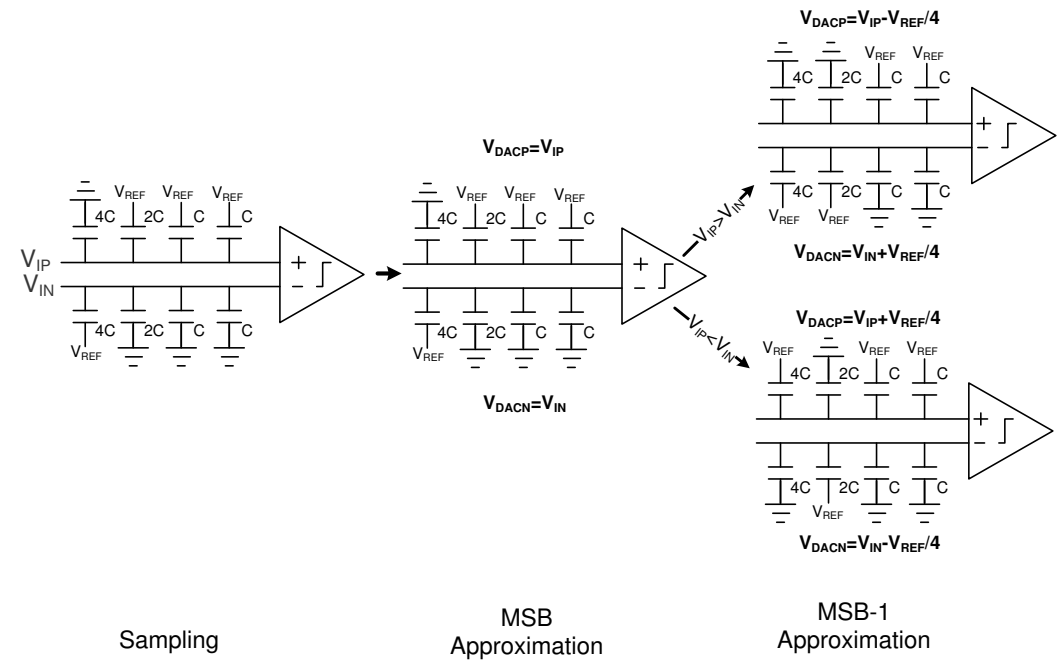

(a)

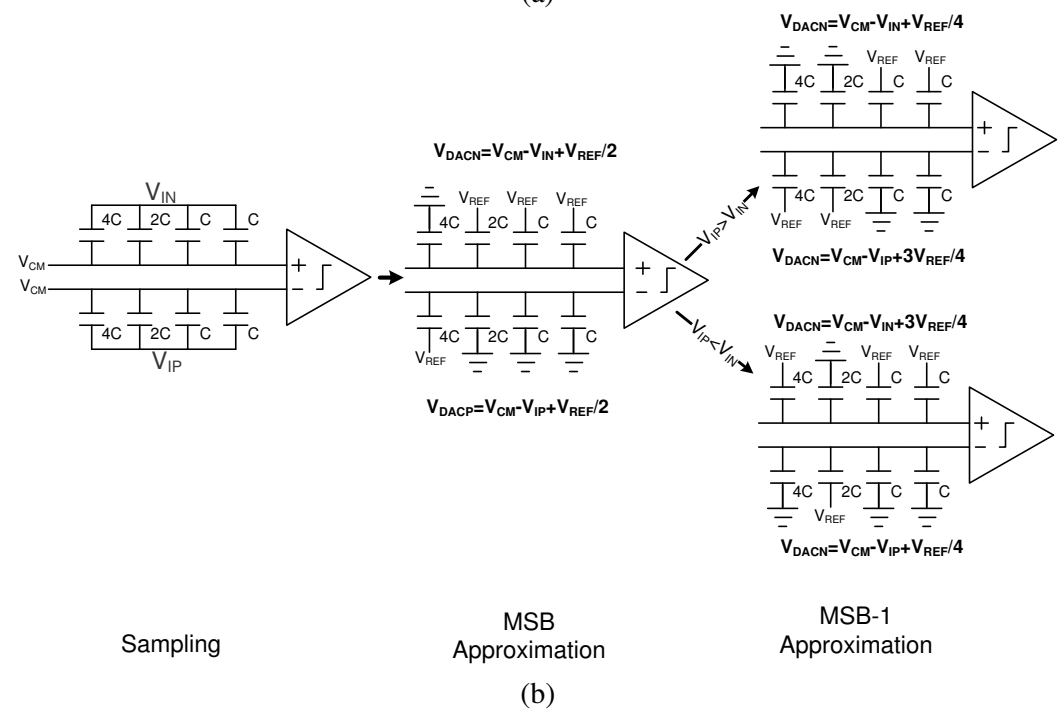

Figure 6.2: The first three DAC switching phases of a 3-bit SAR ADC: (a) employing top-plate sampling; (b) employing bottom-plate sampling. 


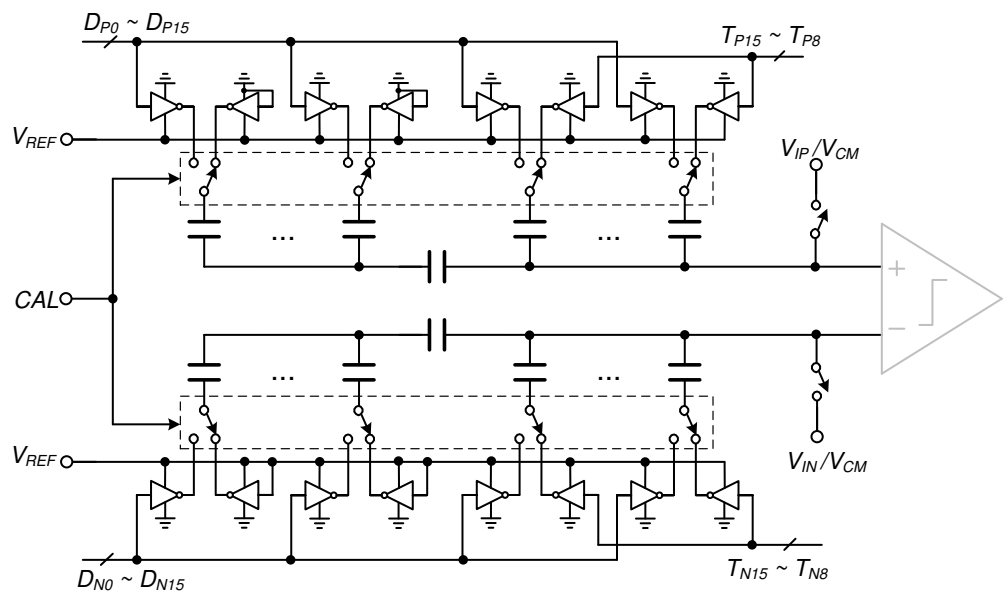

Figure 6.3: The DAC with calibration circuits.

\subsection{Circuit Implementation}

\subsubsection{Capacitive DAC}

In a sub-radix-2 network, it is impossible to dimension the array capacitors with multiples of unit capacitor due to the remaining fractional part. The non-uniform geometry degrades the DAC linearity and requires some form of calibration [39]. In this work, the non-binary-weighted array capacitance is rounded to the nearest integer as shown in Fig. 6.4, thereby leading to a uniform geometry. The dynamic range of the DAC after rounding is guaranteed to be larger than the targeted $14 \mathrm{~b}$ resolution. In addition, a code mapping is performed to ensure no missing code. By employing the secondary-bit approach described in Chapter 5, the ADC has a dynamic error tolerance of $597,322,169, \cdots, 8,8,8 \mathrm{LSBs}$ for the nine most-significant bits.

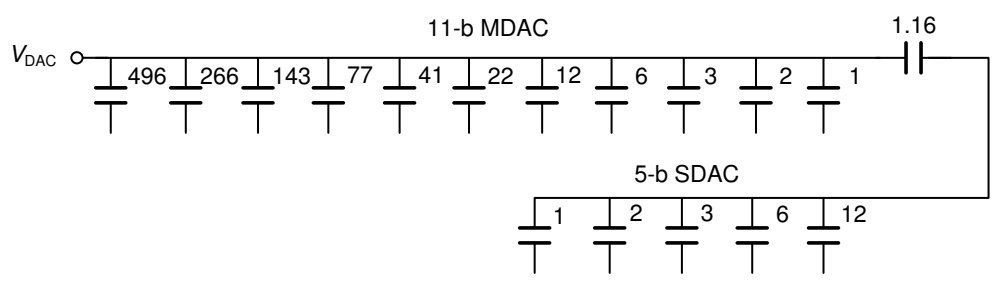

Figure 6.4: Split DAC with its non-binary-weighted numbers rounded to the nearest integer .

A custom-made unit capacitor is implemented to increase the capacitor density, and its sandwich-structure helps to protect the sensitive top-plate node. Its cross 
view is depicted in Fig. 6.5. The unit capacitor is 26.9fF, occupying an active area of $32.5 \mu \mathrm{m}^{2}$. In addition, metal layer 6 is added to create a capacitor of $9.3 \mathrm{fF}$ between the bottom plate and the ground. This additional capacitor helps to reduce the thermal noise introduced by the bottom-plate switches [69]. Part of the DAC noise is band-limited and further reduced by the succeeding comparator. The total sampling capacitor is $28.79 \mathrm{pF}$. The sampling noise causes $2.85-\mathrm{dB}$ loss of SNR for a 14-bit 1-V radix-2 ADC. Furthermore, in order to keep a quiet substrate below the capacitive array, a guard ring of P-substrate, as depicted in Fig. 6.6, is put around the capacitive array.

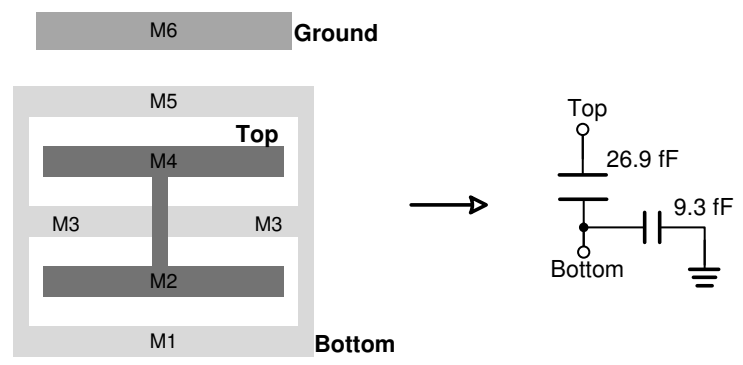

Figure 6.5: Cross view of multi-layer sandwich capacitor and its simplified schemica view.

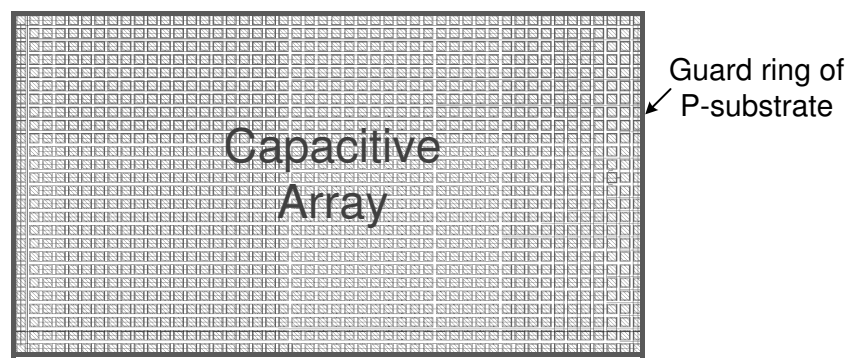

Figure 6.6: Guard ring of P-substrate around the capacitive array for noise isolation.

For a non-binary-weighted split DAC with a radix of $r$, the bridge capacitor, denoted as $C_{b}$, can be approximated to (see Appendix A for the derivation)

$$
C_{b}=\frac{C_{u}}{r-1}
$$

The bridge capacitor is calculated to be $1.16 \times$ bigger than the unit capacitor, and it is intepolated into the array capacitors, as shown in Fig. 6.7. The top-plate of the bridge capacitor is connected to the SDAC to minimize the linearity error introduced by the parasitic capacitance. 


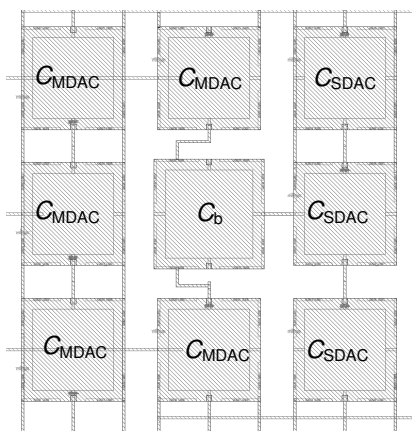

Figure 6.7: Part of the DAC layout showing the connections of the bridge capacitor.

\subsubsection{Comparator}

The block diagram of the comparator is illustrated in Fig. 6.8. A single-pole amplifier (SPA) as the pre-amplification stage proceeds a dynamic latch in order to isolate the latch kickback noise, decrease the input-referred noise, and mitigate the metastability error. In addition, a succeeding SR latch stores the comparison result for an entire clock cycle. To decrease the static power of the SPA, the SPA is switched off once the dynamic latch completes regeneration and starts again at the beginning of the next conversion step.

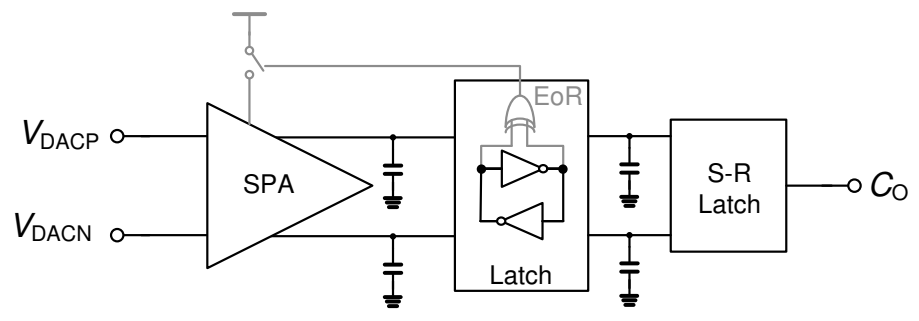

Figure 6.8: Block diagram of the comparator.

A schematic diagram of the preamplifier is shown in Fig. 6.9. The amplifier uses diodeconnected loads and a cross-coupled transistor pair that provides positive feedback for gain enhancement [63]. The DC gain of the amplifier is expressed as

$$
A=\frac{g_{m 1}}{g_{m 3}-g_{m 5}},
$$

where $g_{m 1}, g_{m 3}$, and $g_{m 5}$ respectively refer to the transconductance of the input pair, the diode-connected load pair, and the feedback pair. 


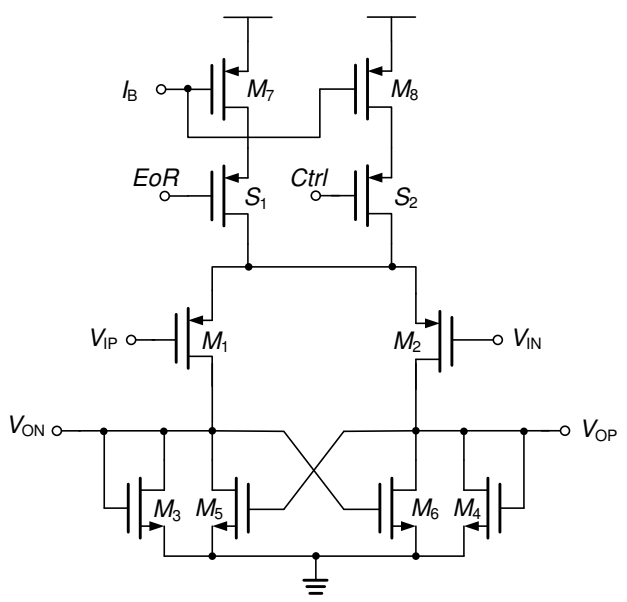

Figure 6.9: Schematic diagram of the preamplifier.

The lower bound of the input transistor transconductance is given by Eq. (2.49) in Sec. 2.3.1.3. For ease of reference, it is copied as follows

$$
g_{m a}=\frac{4 \pi k T \gamma f_{3 d B}}{v_{q n}^{2}}
$$

where $g_{m a}$ is the transconductance of the input transistor, $k$ is the Boltzmann constant, $T$ is the absolute temperature, $\gamma$ is the noise factor, and $v_{q n}^{2}$ is the quantization noise power of $\mathrm{ADC}$. If we further relate $f_{3 d B}$ to the sampling frequency $f_{s}$, we can re-write Eq. (6.3) as

$$
g_{m}=\frac{4 k T \gamma k_{\tau} M f_{s}}{v_{q n}^{2}},
$$

where $k_{\tau}$ is a factor for sufficient settling and $M$ is the number of clock cylces for one conversion. In this work, 17 clock cycles are used for one converstion. Assuming $k_{\tau}$ to be 5 , for 14-bit resolution and $10-\mathrm{kS} / \mathrm{s}$ sampling rate at $1-\mathrm{V}$ full-scale voltage, the minimum $g_{m}$ is calculated to be $45 \mu \mathrm{s} / \mathrm{A}$. Consequently, the input devices are composed of four PMOS devices with $W / L=4 / 1$ and carry about $1.2 \mu \mathrm{A}$ bias current, which results in a simulated $g_{m a}$ of $50 \mu \mathrm{s} / \mathrm{A}$.

The diode-connected loads are composed of three NMOS devices with $W / L=$ $0.3 / 3$. The feedback pair is composed of two NMOS devices with the same size, which triples the DC gain. The simulated DC gain is $9(19 \mathrm{~dB})$. The gain-load product limited by thermal noise is derived in Eq. (2.48) of Sec. 2.3.1.3. For ease of reference, it is copied as follows

$$
A C_{L}=\frac{2 k T \gamma}{v_{q n}^{2}}
$$


The load capacitor $C_{L}$ is chosen to be $2.8 \mathrm{pF}$. The simulated 3-dB bandwidth is $290 \mathrm{kHz}$, and the input-referred noise power of the amplifier is $250 \mathrm{pV}^{2}$, which is lower than the quantization noise power of a $14 \mathrm{~b} 1 \mathrm{~V}$ ADC $\left(310 \mathrm{pV}^{2}\right)$.

Considering the ADC is able to correct early decision errors owing to the redundancy, to further decrease the power consumption half of the bias current is switched off during the conversion of the six most-significant bits (D15 D10) and the sampling phase. The bias transistors, $M_{7}$ and $M_{8}$ in Fig. 6.9, are both composed of eight NMOS devices with $W / L=1 / 1$. When the signal $C t r l$ becomes high, it reduces the bias current by half. Table 6.2 compares the simulated amplifier performance under two bias conditions. The reduction of the bias current hardly affects the DC gain of the pre-amplifier, but it changes the output common-mode voltage. This results in a variation of $0.6 \mathrm{mV}$ to the input-referred offset of the succeeding latch. Further divided by the gain of the preamplifier, the offset variation at the input of the comparator is about 1LSB.

Table 6.2: Comparison of Simulated Amplifier Performance under Two Bias Conditions

\begin{tabular}{|l|c|c|c|}
\hline & Full bias & Half bias & Unit \\
\hline Gain & 19 & 19 & $\mathrm{~dB}$ \\
\hline 3-dB bandwidth & 287 & 195 & $\mathrm{kHz}$ \\
\hline Input transconductance & 50 & 32 & $\mu \mathrm{s} / \mathrm{A}$ \\
\hline Input-referred noise power & 250 & 250 & $\mathrm{pV}^{2}$ \\
\hline Bias current & 2.4 & 1.4 & $\mu \mathrm{A}$ \\
\hline Output common-mode voltage & 516 & 465 & $\mathrm{mV}$ \\
\hline
\end{tabular}

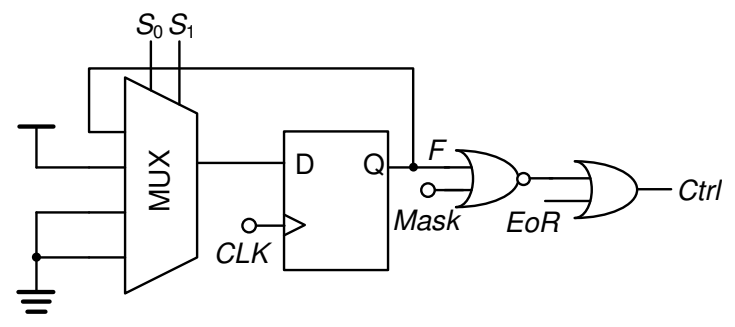

Figure 6.10: Block diagram of the bias control circuit.

Fig. 6.10 depicts the block diagram of the bias control logic and Fig. 6.11 illustrates the corresponding time sequence. Signal $S_{0}$ and $S_{1}$ respectively represent the sampling phase and the conversion phase of $D_{10}$. During this period, half of the bias current is switched off. In addition, the bias control logic can be disabled by setting the Mask signal to High. Based on the transistor-level simulation, the bias-control approach reduces the comparator power by $20 \%$. 


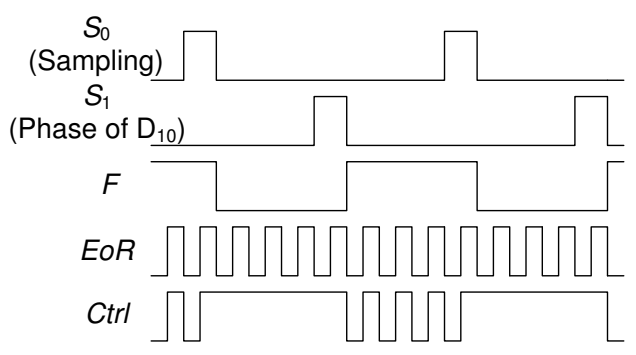

Figure 6.11: Time sequence of the bias control circuit.

Since the pre-amplification stage provides a gain more than 8 , the dynamic latch is designed to have $11 \mathrm{~b}$ resolution with $200-\mathrm{fF}$ load capacitor.

\subsubsection{Biasing Circuit}

Fig. 6.12 shows the schematic view of the biasing circuit [71]. The PMOS pair $M_{1} / M_{2}$ form a first current mirror of gain $S_{1} / S_{2}$. The NMOS pair $M_{3} / M_{4}$ form a second current mirror of gain $S_{4} / S_{3}$. These two current mirrors further form a closed loop, with a loop gain higher than one. If the starting current is small enough so that the voltage drop $V_{R}$ across resistor $R_{S}$ can be neglected. As the current in both branches increases, $V_{R}$ also increases, thereby reducing the loop gain. The equilibrium is finally reached when the loop gain is reduced to one. Biasing the PMOS pair in strong-inversion and NMOS pair in weak-inversion, and assuming the NMOS pair are in the same p-type well, $V_{R}$ at the state of equilibrium can be expressed as [71]

$$
V_{R}=U_{T} \ln \left(\frac{S_{1}}{S_{2}} \cdot \frac{S_{4}}{S_{3}}\right)
$$

where $U_{T}$ is the thermal voltage (about $26 \mathrm{mV}$ at room temperature). Therefore, the eqilibirium current is simply calculated as

$$
I_{R}=\frac{V_{R}}{R_{S}}
$$

In this work, $S_{1} / S_{2}$ is set to be 1 , and $S_{4} / S_{3}$ to be 4 , leading to $V_{R}$ of $36 \mathrm{mV}$ at room temperature. The required resistor is about $200 \mathrm{k} \Omega$, and it is off-chip so that tuning during measurement is possible. Additionally, the current biasing circuit can be disabled by switching the enable signal En from Low to High. Consequently, an external bias voltage can be applied to the gate of the tail transistors in the preamplifier through a transmission gate. 


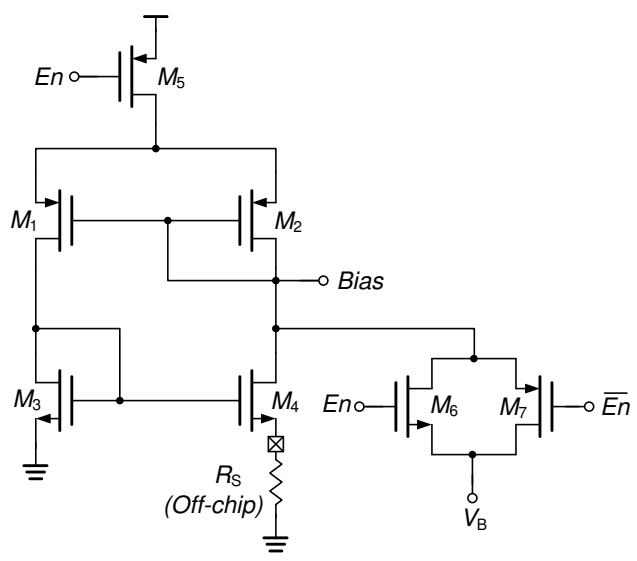

Figure 6.12: Schematic view of the biasing circuit.

\subsubsection{Sampling Switch}

Fig. 6.13 shows the well-known gate-source bootstrapping technique [72]. It comprises an NMOS sampling device, a capacitor, and five switches controlled by two non-overlapping clock phases. The detailed working procedure of the bootstrapping circuit is illustrated in Fig. 6.14. At $\Phi_{1}$, the gate of the sampling device is reset to ground, and the capacitor is charged to $V_{D D}$. At $\Phi_{2}$, the precharged capacitor is in series with the input voltage, applying a gate-source voltage about $V_{D D}$ to the sampling device. Hence, it guarantees that the sampling condunctance is independent of the input signal.

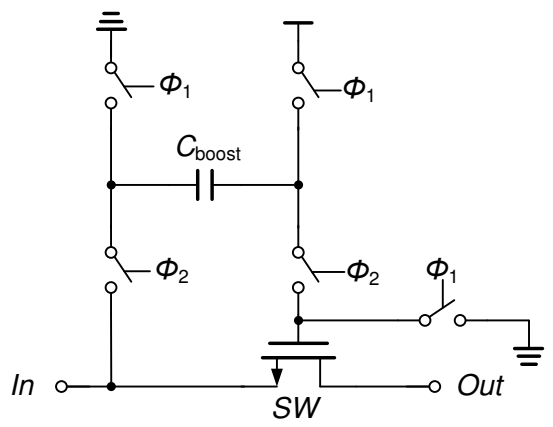

Figure 6.13: Simplified gate-source bootstrapping circuit.

The transistor-level implementation [73] is shown in Fig. 6.15. $M_{N 1}, M_{N 2}, M_{N 6}$, $M_{P 2}$, and $M_{P 3}$ correspond to the five ideal switches shown in Fig. 6.13. At $\Phi_{1}, M_{N 1}$ 


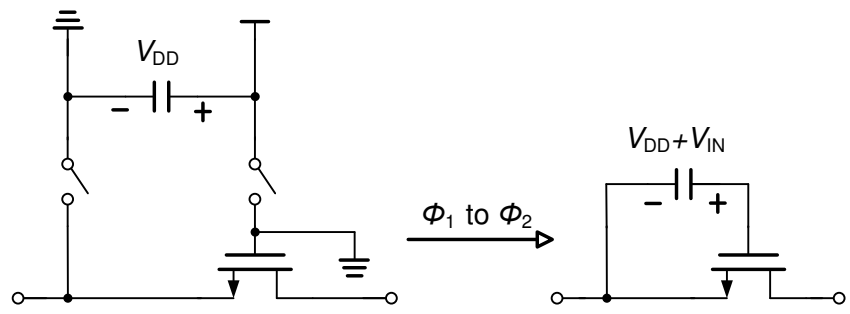

Figure 6.14: Two phases of gate-source bootstrapping circuit.

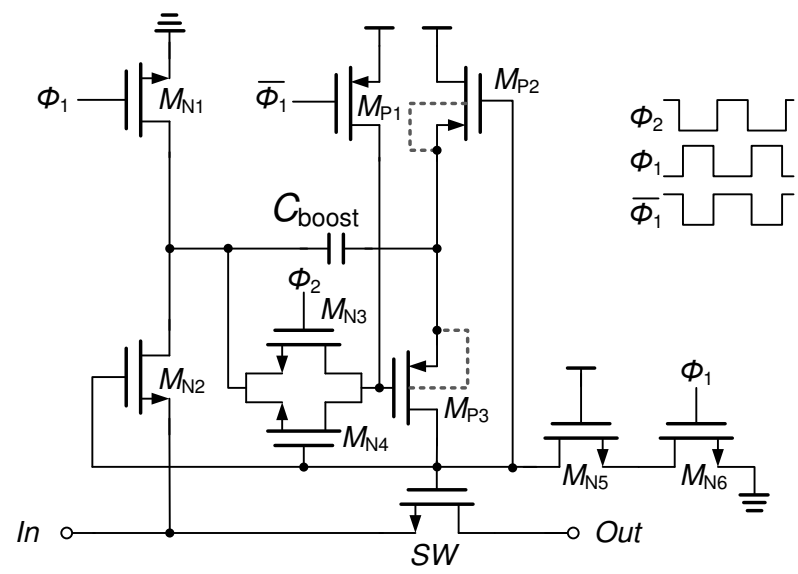

Figure 6.15: Schematic view of the bootstrapping circuit [73]. 
and $M_{P 2}$ is turned on, precharging the capacitor. At $\Phi_{2}, M_{N 3}$ acts as a start-up device to force $M_{P 3}$ to conduct. Then, $M_{P 2}$ is also turned on. The gate voltage of the sampling NMOS transistor is finally boosted to the sum of the input voltage and the supply voltage. In addition, transistor $M_{N 5}$ is added in series with $M_{N 6}$ to prevent the gate-drain voltage of the latter from reaching $2 V_{D D}$.

In this work, the capacitor is set to be $180 \mathrm{fF}$, and the sampling device is sized with $1 / 0.12$. The differential sampling circuit with $29-\mathrm{pF}$ capacitive load was simulated and the sampled differential voltages were recorded and performed in FFT. The worst-case SNDR is $104 \mathrm{~dB}$ under $80{ }^{\circ} \mathrm{C}$ at the slow process corner.

\subsubsection{Digital Control Logic}

The block diagram of the SAR control logic is illustrated in Fig. 6.16. It consists of a shift register, a set of postive-transparent latch, and a combinational switching logic. The shifter register generates approximation pulses, the latch stores the comparator decision, and the combinational logic generates control signals for the bottom-plate switches of the differential DAC. Total 17 clock cycles are required to complete one conversion: one for sampling and sixteen for bit decision.

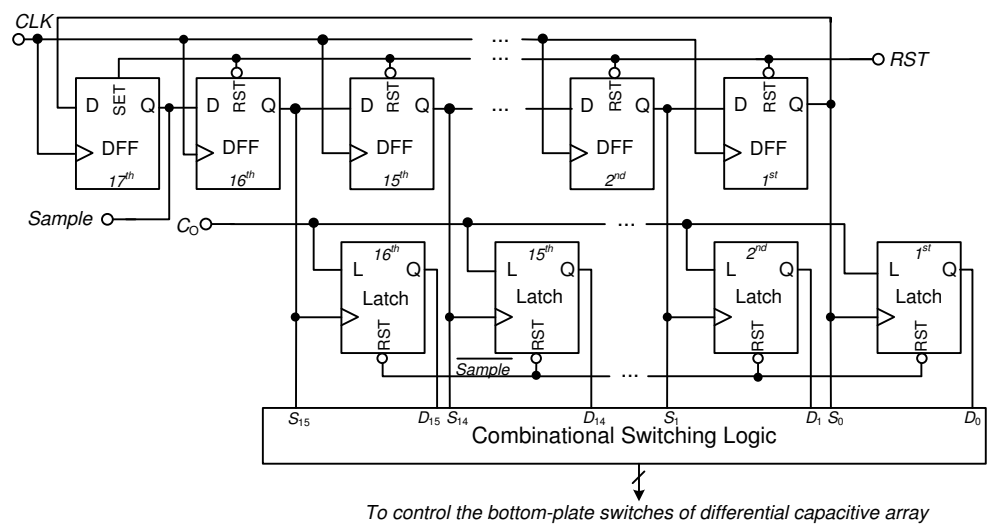

Figure 6.16: Block diagram of SAR control logic.

In Fig. 6.17, signals $S_{X}$ and $D_{X}$ are generated in the shift register and the latch, respectively; signals $N_{X}$ and $P_{X}$ are applied as control signals to the bottom-plate switching bank of the differential DAC. Two types of combinational gate are used: Type (a) shown in Fig. 6.17 is for the higher-weighted bits from $D_{15}$ to $D_{12}$ and the lower-weighted bits from $D_{3}$ to $D_{0}$; Type (b) is for the rest of the bits from $D_{11}$ to $D_{4}$. The secondary-bit approach for dynamic comparison threshold is applied till bit $D_{8}$. 


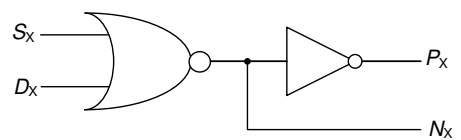

(a)

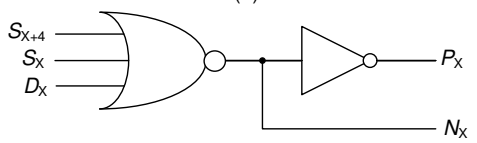

(b)

Figure 6.17: Gates used in the combinational switching logic.

\subsection{Measurement Results}

The prototype SAR ADC with a core area of $530 \times 530 \mu \mathrm{m}^{2}$ was fabricated in $65 \mathrm{~nm}$ CMOS process. It was packaged in a $1.27 \mathrm{~mm}$ pitch JLCC package. A photograph of the chip is shown in Fig. 6.18. The unmarked part around the ADC core includes the decoupling capacitors and the I/O buffers for the pads.

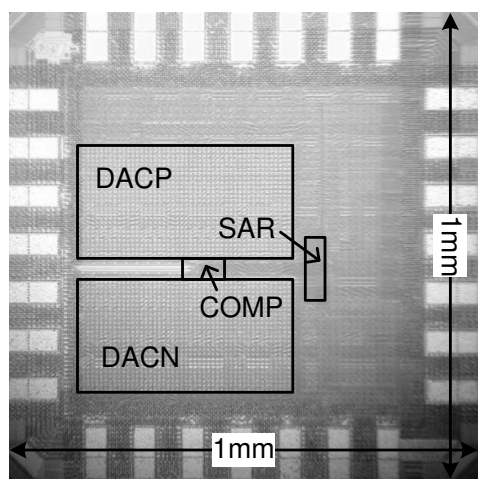

Figure 6.18: Chip photo of the ADC in 65nm CMOS technology.

Histogram test was conducted to measure the linearity of the ADC. A full-swing, differential sinusoidal input near DC frequency with amplitude of $1-\mathrm{V}$ was applied to the 10-kS/s ADC. Fig. 6.19 shows the measured DNL and INL error before and after calibration. Before calibration, the peak DNL error is +1.47/-0.64 LSB, and the peak INL error is $+1.63 /-2.77$ LSB. While after calibration, the peak DNL error changes to $+1.48 /-0.63 \mathrm{LSB}$, and the peak INL error to +0.88/-2.54 LSB. The calibration doesn't show much improvement of the ADC linearity, which indicates that the ADC is noiselimited instead of mismatch-limited. In view of this, the following measurement was performed on the ADC with the calibration disabled. 

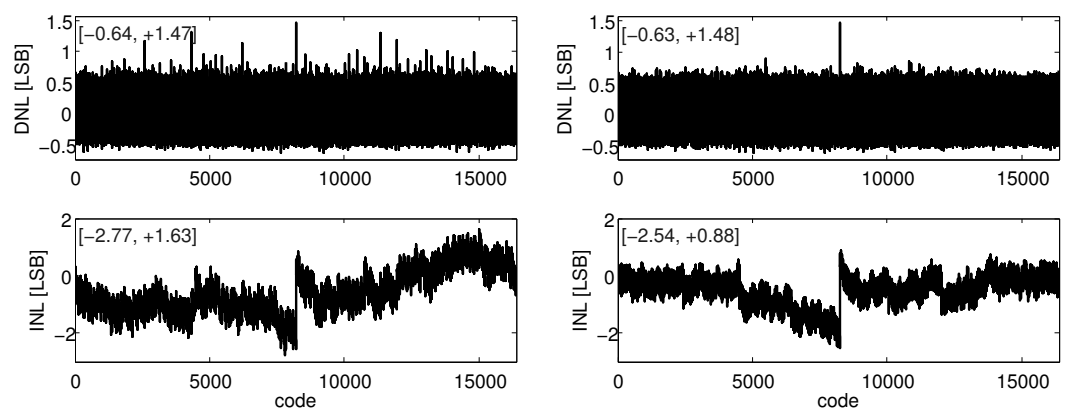

(a)

(b)

Figure 6.19: Measured DNL and INL error according to 14-bit resolution at $1 \mathrm{~V}$ : (a) before calibration (b) after calibration.

At 1-V supply, the FFT spectrums of the 10-kS/s ADC at near DC and Nyqsuit operations are depcited in Fig. 6.20. The amplitude of the test stimulus was set to be $-0.33 \mathrm{dBFS}$. The measured SNDR near Nyquist is $77.5 \mathrm{~dB}$, providing 12.6 ENOB. Fig. 6.21 shows the SFDR, SNR, and SNDR with respect to the input frequency. The SNDR remains almost constant over the entire bandwidth.

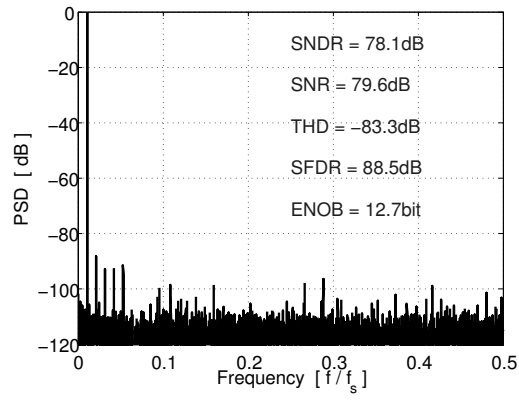

(a)

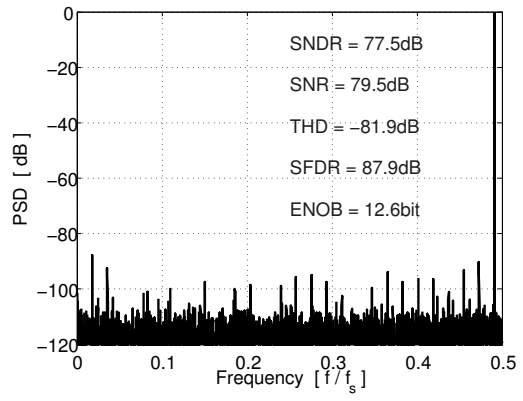

(b)

Figure 6.20: Measured 16384-point FFT spectrums of 10-kS/s 1-V ADC: (a) near DC (b) near Nyquist.

Reducing the supply voltage is an effective way to reduce the power consumption. Hence, the ADC performance was also measured at 0.8-V supply. The FFT spectrums are depcited in Fig. 6.22. The measured SNDR near Nyquist is $77.0 \mathrm{~dB}$, providing 12.5 ENOB. Fig. 6.23 shows the SFDR, SNR, and SNDR with respect to the input frequency. The static performance is shown in Fig. 6.24. The peak DNL error is $+2.28 /-0.90 \mathrm{LSB}$, and the peak INL error is +2.22/-2.26 LSB. 


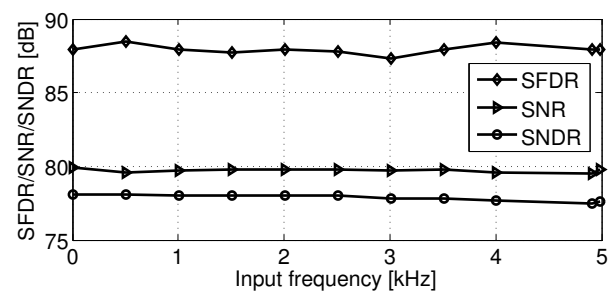

Figure 6.21: SFDR, SNR, and SNDR versus input frequency at $1 \mathrm{~V}$.

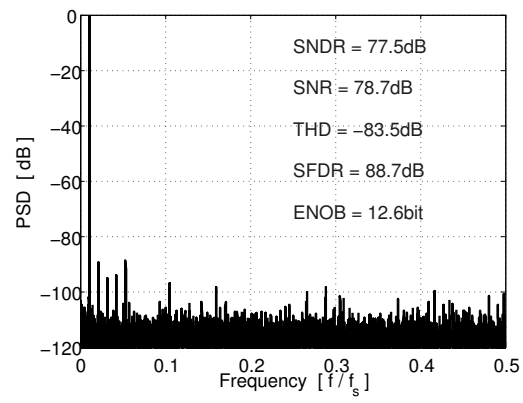

(a)

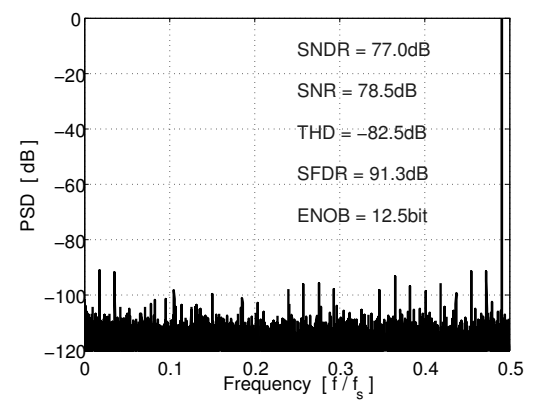

(b)

Figure 6.22: Measured 16384-point FFT spectrums of 10-kS/s 0.8-V ADC: (a) near DC (b) near Nyquist.

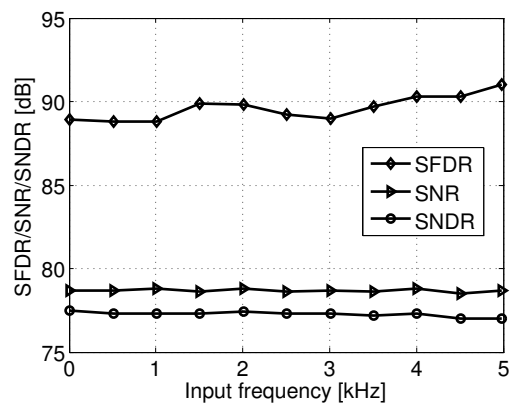

Figure 6.23: SFDR, SNR, and SNDR versus input frequency at $0.8 \mathrm{~V}$. 

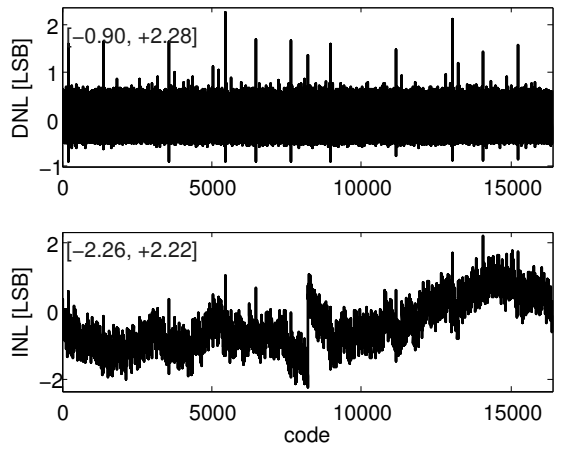

Figure 6.24: Measured DNL and INL error according to 14-bit resolution at $0.8 \mathrm{~V}$.

Table 6.3 shows the measured power consumption of the entire ADC as well as its individual blocks at two different supply voltages. The ADC consumes $2.48 \mu \mathrm{W}$ and $1.98 \mu \mathrm{W}$ at $1 \mathrm{~V}$ and $0.8 \mathrm{~V}$, respectively. About $78 \%$ of the total power is dissipated in the comparator, $20 \%$ in the DAC, and $2 \%$ in the digital control logic. During the calibration mode, the test logic only consumes $3 \mathrm{nW}$ at $1 \mathrm{~V}$. Table 6.5 summerizes the ADC performance.

Table 6.3: Measured ADC Power Consumption

\begin{tabular}{|l|c|c|}
\hline & Power at $1 \mathrm{~V}$ & Power at $0.8 \mathrm{~V}$ \\
\hline Comparator $[\mu \mathrm{W}]$ & 1.888 & 1.592 \\
\hline DAC $[\mu \mathrm{W}]$ & 0.549 & 0.356 \\
\hline SAR $[\mu \mathrm{W}]$ & 0.047 & 0.030 \\
\hline Total $[\mu \mathrm{W}]$ & 2.48 & 1.98 \\
\hline
\end{tabular}

Two scatter plots of FOM (Walden FOM and Thermal FOM) versus SNDR of previously reported Nyquist SAR ADCs [7] together with this work are shown in Fig. 6.25. This ADC achieves the highest SNDR compared to other Nyquist SAR ADCs. Table 6.5 provides a more detailed comparison between this ADC and other works with comparable accuracy.

It will be interesting to see the position of this ADC in Fig. 4.29 of Chapter 4, where the predicted mismatch-limited energy bounds together with Nyquist SAR ADC survery data from [7] and the previous two works are plotted. It is updated in Fig. 6.26. The three ADCs continue to approach the predicted bounds. 
Table 6.4: ADC Measurement Summary

\begin{tabular}{|l|c|c|}
\hline Technology & \multicolumn{2}{|c|}{ 65nm CMOS } \\
\hline Core area $\left[\mathrm{mm}^{2}\right]$ & \multicolumn{2}{|c|}{0.28} \\
\hline Resolution [bits] & \multicolumn{2}{|c|}{14} \\
\hline Input range & \multicolumn{2}{|c|}{$0-V_{D D}$} \\
\hline Sampling rate [kS/s] & \multicolumn{2}{|c|}{0.8} \\
\hline Supply voltage [V] & 1 & $+2.28 /-0.90$ \\
\hline DNL [LSB] & $+1.47 /-0.64$ & $+2.22 /-2.26$ \\
\hline INL [LSB] & $+1.63 /-2.77$ & +77.0 \\
\hline SNDR (at Nyquist) [dB] & 77.5 & 91.3 \\
\hline SFDR (at Nyquist) [dB] & 87.9 & 12.5 \\
\hline ENOB [bits] & 12.6 & 1.98 \\
\hline Total Power $[\mu \mathrm{W}]$ & 2.48 & 34.2 \\
\hline FOMW ${ }^{\dagger}[\mathrm{fJ} /$ conv.step] & 39.9 & 171.0 \\
\hline FOMS ${ }^{\ddagger}[\mathrm{dB}]$ & 170.5 & \\
\hline
\end{tabular}

${ }^{\dagger}$ Walden FOM $=\frac{\text { Power }}{2^{E N O B} f_{s}}$

${ }^{\ddagger}$ Thermal FOM $=S N D R+10 \log _{10} \frac{B W}{\text { Power }}$
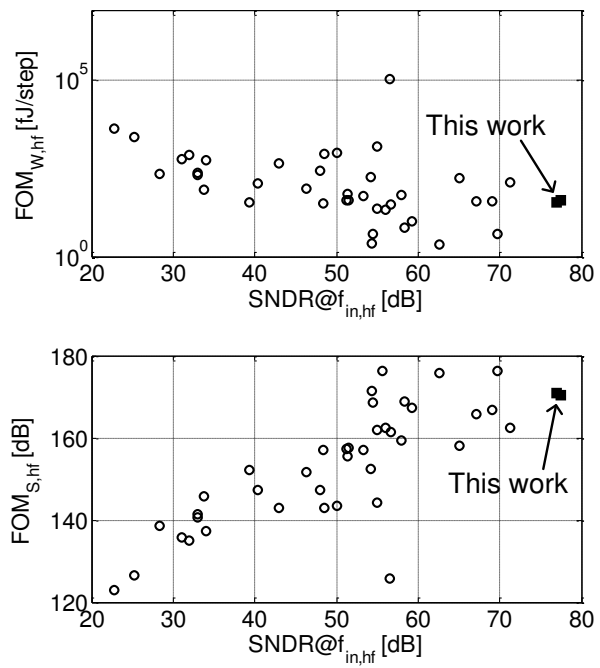

Figure 6.25: Walden FOM and thermal FOM versus SNDR@Nyquist of Nyquist SAR ADCs presented at ISSCC from data in [7]. 
Table 6.5: ADC Comparison

\begin{tabular}{|c|c|c|c|c|c|c|c|}
\hline & [74] & [70] & [75] & [69] & [76] & \multicolumn{2}{|c|}{ This work } \\
\hline Technology [nm] & $500 / 250$ & 65 & 130 & 90 & 65 & \multicolumn{2}{|c|}{65} \\
\hline Architecture & $\begin{array}{c}\text { SAR-assisted } \\
\text { pipelined }\end{array}$ & $\begin{array}{c}\text { Oversampled } \\
\text { SAR }\end{array}$ & $\begin{array}{c}\text { Nyquist } \\
\text { SAR }\end{array}$ & $\begin{array}{c}\text { Nyquist } \\
\text { SAR }\end{array}$ & $\begin{array}{c}\text { Nyquist } \\
\text { SAR }\end{array}$ & \multicolumn{2}{|c|}{$\begin{array}{l}\text { Nyquist } \\
\text { SAR }\end{array}$} \\
\hline Area $\left[\mathrm{mm}^{2}\right]$ & - & 0.18 & 0.059 & 0.097 & 0.55 & \multicolumn{2}{|c|}{0.28} \\
\hline Resolution [bit] & 18 & 14 & 12 & 13 & 14 & \multicolumn{2}{|c|}{14} \\
\hline Sampling rate $[\mathrm{S} / \mathrm{s}]$ & $12.5 \mathrm{M}$ & $128 \mathrm{k}$ & $22.5 \mathrm{M}$ & $50 \mathrm{M}$ & $80 \mathrm{M}$ & \multicolumn{2}{|c|}{$10 \mathrm{k}$} \\
\hline Supply voltage [V] & $5 / 2.5$ & 0.8 & 1.2 & 1.2 & 1.2 & 1 & 0.8 \\
\hline DNL [LSB] & 0.5 & - & - & - & - & 1.5 & 2.3 \\
\hline INL [LSB] & 2.2 & - & - & - & - & 2.8 & 2.3 \\
\hline SFDR $[\mathrm{dB}]$ & 82.0 & 87.1 & 90.3 & 84.0 & 80.3 & 87.3 & 88.8 \\
\hline SNDR [dB] & 80.0 & $79.1(\times 16$ OSR $)$ & 71.1 & 69.1 & 71.3 & 77.5 & 77.0 \\
\hline Power $[\mathrm{W}]$ & $105 \mathrm{~m}$ & $1.37 \mu$ & $3 \mathrm{~m}$ & $4.2 \mathrm{~m}$ & $31.1 \mathrm{~m}$ & $2.48 \mu$ & $1.98 \mu$ \\
\hline FOMW [fJ/conv.step] & 1027.8 & 23.2 & 50.8 & 36.1 & 129.5 & 39.9 & 34.2 \\
\hline FOMS $[\mathrm{dB}]$ & 157.7 & 173.8 & 165.9 & 166.8 & 162.4 & 170.5 & 171.0 \\
\hline
\end{tabular}

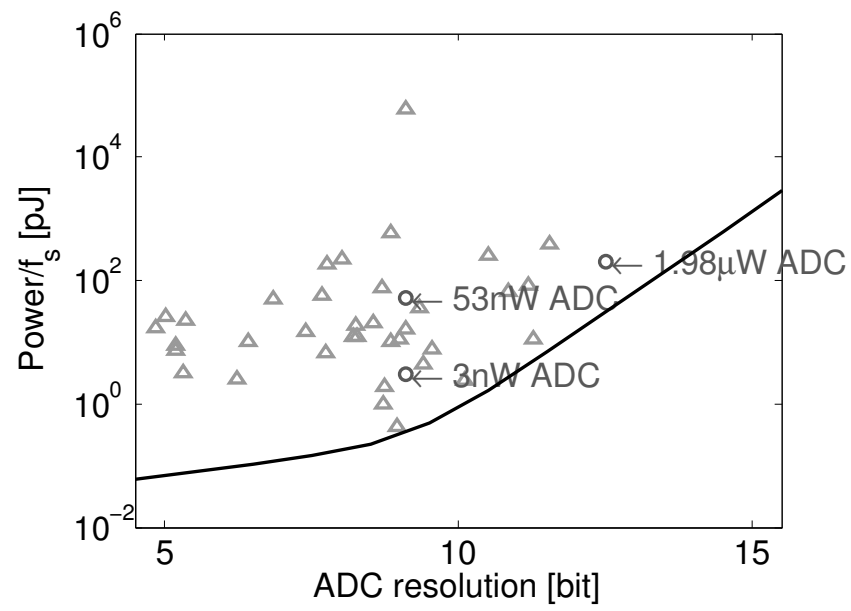

Figure 6.26: Predicted mismatch-limited SAR ADC energy bounds (solid line) together with Nyquist SAR ADC survey data $(\Delta)$ and the implemented ADCs (o). 


\section{Chapter 7}

\section{Conclusions and Future Directions}

\subsection{Conclusions}

Biomedical systems require low-speed ADCs with ultra-low-power operation. Among prevalent ADC architectures, SAR ADCs are favored due to their high energy efficiency. The error sources as well as the power consumption bounds of the SAR ADC have been discussed in Chapter 2 and Chapter 3, respectively. At low resolution, the power consumption is bounded by digital switching power. At medium-to-high resolution, the power consumption is bounded by thermal noise and capacitor mismatch.

Based on the understanding from the power analysis, two 10 bit $1 \mathrm{kS} / \mathrm{s}$ SAR ADCs, following the design strategy with maximum simplicity in the architecture, have been presented in Chapter 4. The first ADC, implemented in a $0.13 \mu \mathrm{m}$ CMOS process, achieves 9.1 ENOB with 53-nW power consumption by using a binary-weighted capacitive DAC with a top-plate sampling technique, a dual-supply voltage scheme allowing the SAR control logic to operate at $0.4 \mathrm{~V}$, as well as low-leakage circuit techniques and considerable design optimization. The leakage power constitutes $25 \%$ of the total power consumption. The second ADC, implemented in a $65 \mathrm{~nm}$ CMOS process, achieves 9.1 ENOB and makes a substantial (94\%) improvement in the power consumption, resulting in 3-nW total power. The ultra-low-power consumption is achieved by using a small split-array capacitive DAC, a bottom-plate sampling approach reducing charge injection error and allowing full-range input sampling without extra voltage sources, and a latch-based SAR control logic resulting in reduced power and low transistor count. Furthermore, a multi- $\mathrm{V}_{\mathrm{T}}$ circuit design approach allows the ADC to meet the target performance with a single supply voltage 
of $0.7 \mathrm{~V}$. The ADC can even operate down to a supply voltage of $0.6 \mathrm{~V}$, achieving an optimal energy efficiency of $4.5 \mathrm{fJ} /$ conversion-step with $8.8 \mathrm{ENOB}$ at $1 \mathrm{kS} / \mathrm{s}$.

In order to relax the mismatch requirement on the capacitor sizing while still ensuring enough linearity for high resolution, a bottom-up weight calibration technique has been proposed in Chapter 5. It utilizes redundancy generated by a non-binaryweighted capacitive network, and measures the actual weights of more significant capacitors using less significant capacitors. In addition, the effect of capacitor variation on the design of non-binary-weighted capacitive DACs has been analyzed. It shows that a larger capacitor variation requires a smaller radix and needs more conversion steps. Simulations based on a behavioral model of a 15-bit 1.85-radix ADC demonstrates that both SNDR and DNL/INL are improved after calibration.

In Chapter 6, a 14 bit $10 \mathrm{kS} / \mathrm{s}$ redundant SAR ADC in 65nm CMOS process has been presented. The ADC implements a uniform-geometry non-binary-weighted capacitive DAC for linearity enhancement and employs a secondary-bit approach to dynamically shift decision levels for error correction. Moreover, a comparator with bias control utilizes the redundancy to reduce the power consumption. The calibration technique proposed in Chapter 5 is applied to the ADC as well. However, since the ADC turns out to be noise-limited instead of mismatch-limited, the calibration doesn't help to improve the ADC performance much. Measurement results show that the ADC without calibration achieves $77 \mathrm{~dB}$ SNDR up to Nyquist with $1.98 \mu \mathrm{W}$ at $0.8 \mathrm{~V}$.

\subsection{Future Directions}

In order to design ultra-low-power SAR ADC, we start by investigating the error sources which limit the conversion accuracy, then analyze the ADC power bounds. Based on the understanding from the power analysis, the two ADCs presented in Chapter 4 achieve power consumption within nano-watt range. We have learned that imposing maximal simplicity in the ADC architecture and low transistor count in the digital logic is an efficient way to reduce the power consumption. However, there could be an alternative approach, which takes advantage of the fact that biomedical signals often exhibit small variations in magnitude for a large portion of time, such as ECG signal shown in Fig. 7.1. The burst-like property of the signal indicates that traditional successive approximation algorithm, which always starts conversion from the MSB and continues towards the LSB, may not be efficient enough compared to signal-activity-based algorithms [77, 78]. In [77], the ADC selects switching sequences to skip several conversion steps when the signal is within a predefined small window. In [78], the ADC uses a previous conversion result as the initial guess of the current sample and bit-cylces the LSBs first. However, there is a trade-off between complex algorithm and simple circuit. The optimum performance of such data-dependent energy-saving techniques applied to the SAR architecture is worthy of further investigation. 


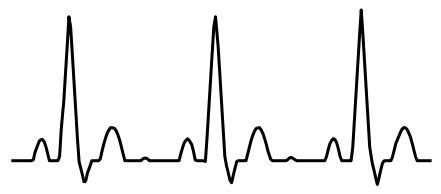

Figure 7.1: ECG signal.

Compared to other Nyquist ADCs, the SAR ADC can generate conversion residue without a feedback DAC. This special property can be utilized to apply noise shaping to the SAR architecture [79, 80]. As shown in Fig. 7.2, a capacitor denoted as $C_{R}$ is added besides the DAC array in order to sample the conversion residue, and the residue is further integrated. Applying the output of the integrator to the opposite input of the comparator, the corresponding linear model of an entire SAR ADC is illustrated in Fig. 7.3. It can be seen that the first-order noise shaping is achieved. However, there are practical issues degrading the efficiency of noise-shaping, such as the $\mathrm{kT} / \mathrm{C}$ noise introduced during the residue sampling and the finite amplifier gain of the integrator. In order to achieve high resolution, the residue sampling capacitor should be sized large enough to mitigate the thermal noise. In addition, the gain of the amplifier should be enhanced to ensure an integrator with good quality factor. This extra requirement makes the SAR ADC not amenable to scaling anymore. Nevertheless, the potential of noise-shaping technique applied to the SAR architecture has certainly not been fully explored. More efforts are needed to efficiently utilize this technique to further enhance the accuracy and lower the conversion energy of the SAR ADC.

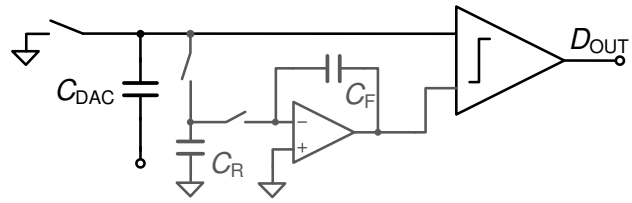

Figure 7.2: An additional capacitor and an integrator are added to the SAR architecture to perform 1st-order noise-shaping.

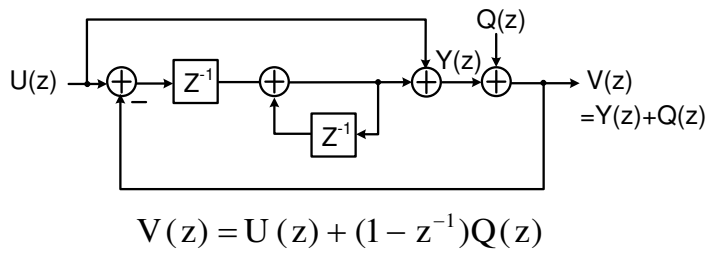

Figure 7.3: Linear model transfer functions of a 1st-order noise-shaping SAR ADC. 


\section{Appendix A}

\section{Calculation of the Bridge Capacitor (Eq. (6.1))}

The capacitors in both main-DAC and sub-DAC has the radix of $r$. The capacitors at the two terminals of the bridge capacitor should also follow the same radix, which further indicates the ratio of their corresponding weight is equal to $r$. As shown in Fig. A.1, the two consecutive weights are indicated as $W_{j}$ and $W_{i}$, respectively. Denoting the total MDAC capacitor as $C_{m}$ and SDAC capacitor as $C_{s}$, weight $W_{j}$ can be calculated as

$$
W_{j}=\frac{C_{u}}{C_{m}+C_{b} / / C_{s}} \approx \frac{C_{u}}{C_{m}+C_{b}},
$$

where $C_{b} / / C_{s}$ stands for the series connection of the two capacitor, and it is equal to

$$
C_{b} / / C_{s}=\frac{C_{b} C_{s}}{C_{b}+C_{s}} .
$$

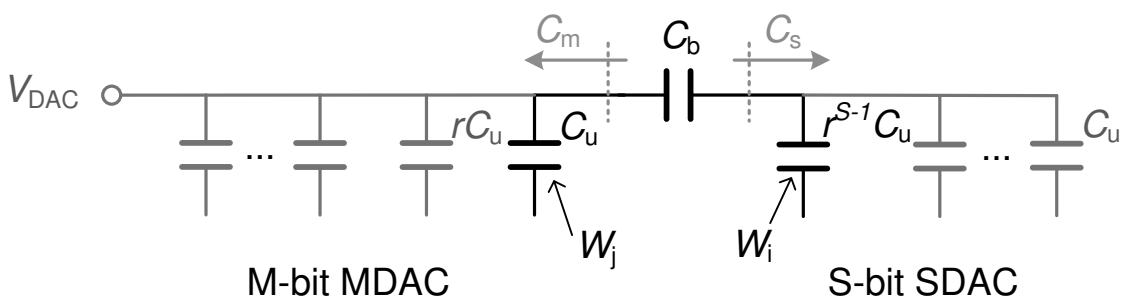

Figure A.1: Split capacitive DAC with one bridge capacitor. 
Moving to the weight $W_{i}$, it can be calculated as

$$
W_{i}=\frac{r^{S-1} C_{u}}{C_{b} / / C_{m}+C_{s}} \frac{C_{b}}{C_{b}+C_{m}} \approx \frac{r^{S-1} C_{u}}{C_{b}+C_{s}} \frac{C_{b}}{C_{b}+C_{m}} .
$$

The ratio between $W_{j}$ and $W_{i}$ is equal to $r$, which leads to

$$
\frac{W_{j}}{W_{i}}=\frac{C_{u}}{r^{S-1} C_{u}}\left(1+\frac{C_{s}}{C_{b}}\right)=r .
$$

Finally, the bridge capacitor can be calculated from Eq. (A.4) as

$$
C_{b}=\frac{C_{s}}{r \frac{r^{S-1} C_{u}}{C_{u}}-1}=\frac{C_{s}}{r^{S}-1}=\frac{C_{u}}{r-1} .
$$




\section{Appendix B}

\section{Paper Collections}

\section{Journals}

- Dai Zhang, Ameya Bhide, and Atila Alvandpour, "A 53-nW 9.1-ENOB 1-kS/s SAR ADC in 0.13- $\mu \mathrm{m}$ CMOS for Medical Implant Devices", in IEEE Journal of Solid-State Circuits, vol.47, no.7, pp.1585-1593, July, 2012.

- Dai Zhang and Atila Alvandpour, "Analysis and Calibration of Non-BinaryWeighted Capacitive DAC for High-Resolution SAR ADCs", accepted for publication in IEEE Transactions on Circuits and System - II: Express Briefs.

\section{Conferences}

- Dai Zhang and Atila Alvandpour, "A 3-nW 9.1-ENOB SAR ADC at 0.7 V and $1 \mathrm{kS} / \mathrm{s}$ ", accepted for publication in proceedings of the European Solid-State Circuit Conference (ESSCIRC), Bordeaux, France, September 2012.

- Dai Zhang, Christer Svensson, and Atila Alvandpour, "Power consumption bounds for SAR ADCs", in proceedings of the European Conference on Circuit Theory and Design (ECCTD), pp.556-559, Linköping, Sweden, August 2011.

- Dai Zhang, Ameya Bhide, and Atila Alvandpour, "Design of CMOS sampling switch for ultra-low power ADCs in biomedical applications", in proceedings of the Norchip Conference, pp.1-4, Tempera, Finland, November 2010. 


\section{Paper Collection}

The articles associated with this thesis have been removed for copyright reasons. For more details about these see:

http://urn.kb.se/resolve?urn=urn:nbn:se:liu:diva-110387 


\section{References}

[1] R. F. Yazicioglu, P. Merken, R. Puers, and C. Van Hoof. A $60 \mu \mathrm{W} 60 \mathrm{nV} / \mathrm{hz}$ readout front-end for portable biopotential acquisition systems. IEEE Journal of Solid-State Circuits, 42(5):1100-1110, 2007.

[2] R. F. Yazicioglu, Sunyoung Kim, T. Torfs, Hyejung Kim, and C. Van Hoof. A $30 \mu \mathrm{w}$ analog signal processor ASIC for portable biopotential signal monitoring. IEEE Journal of Solid-State Circuits, 46(1):209-223, 2011.

[3] A. C.-W. Wong, D. McDonagh, G. Kathiresan, O. C. Omeni, O. El-Jamaly, T. C.K. Chan, P. Paddan, and A. J. Burdett. A 1V, micropower system-on-chip for vital-sign monitoring in wireless body sensor networks. In IEEE International Solid-State Circuits Conference Digest of Technical Papers (ISSCC), pages 138-139, 2008.

[4] Hua Gao, R.M. Walker, P. Nuyujukian, K.A.A. Makinwa, K.V. Shenoy, B. Murmann, and T.H. Meng. HermesE: A 96-channel full data rate direct neural interface in $0.13 \mu \mathrm{m}$ CMOS. IEEE Journal of Solid-State Circuits, 47(4):1043 -1055, April 2012.

[5] R. Sarpeshkar, C. Salthouse, Ji-Jon Sit, M.W. Baker, S.M. Zhak, T.K.-T. Lu, L. Turicchia, and S. Balster. An ultra-low-power programmable analog bionic ear processor. IEEE Transactions on Biomedical Engineering, 52(4):711 -727, April 2005.

[6] J. G. Webster. Medical Instrumentation Application and Design. Wiley, 1998.

[7] B. Murmann, ADC performance survey 1997-2014, [Online]. http: / /www . stanford.edu/murmann/adcsurvey.html.

[8] Sheng-Jui Huang and Yung-Yu Lin. A $1.2 \mathrm{~V} 2 \mathrm{MHz}$ bw $0.084 \mathrm{~mm}^{2} \mathrm{CT} \Delta \Sigma$ ADC with $-97.7 \mathrm{dBc}$ THD and $80 \mathrm{~dB}$ dr using low-latency DEM. In IEEE International Solid-State Circuits Conference Digest of Technical Papers (ISSCC), pages 172-173, 2009. 
[9] A.P. Perez, E. Bonizzoni, and F. Maloberti. A 84dB SNDR 100kHz bandwidth low-power single op-amp third-order $\Delta \Sigma$ modulator consuming $140 \mu \mathrm{w}$. In IEEE International Solid-State Circuits Conference Digest of Technical Papers (ISSCC), pages $478-480$, Feb. 2011.

[10] P. Witte, J.G. Kauffman, J. Becker, Y. Manoli, and M. Ortmanns. A 72dbDR $\Delta \Sigma$ CT modulator using digitally estimated auxiliary DAC linearization achieving $88 \mathrm{fJ} /$ conv in a $25 \mathrm{MHz}$ BW. In IEEE International Solid-State Circuits Conference Digest of Technical Papers (ISSCC), pages 154-156, Feb. 2012.

[11] Tsung-Che Lu, Lan-Da Van, Chi-Sheng Lin, and Chun-Ming Huang. A 0.5V $1 \mathrm{KS} / \mathrm{s} 2.5 \mathrm{nW}$ 8.52-ENOB 6.8fJ/conversion-step SAR ADC for biomedical applications. In IEEE Custom Integrated Circuits Conference (CICC), pages $1-4,2011$.

[12] M. Yip and A.P. Chandrakasan. A resolution-reconfigurable 5-to-10b 0.4-to$1 \mathrm{~V}$ power scalable SAR ADC. In IEEE International Solid-State Circuits Conference Digest of Technical Papers (ISSCC), pages 190 -192, Feb. 2011.

[13] Pieter Harpe, Eugenio Cantatore, and Arthur van Roermund. A 2.2/2.7fJ/conversion-step 10/12b 40kS/s SAR ADC with data-driven noise reduction. In IEEE International Solid-State Circuits Conference Digest of Technical Papers (ISSCC), pages 270-271, 2013.

[14] A. Shikata, R. Sekimoto, T. Kuroda, and H. Ishikuro. A $0.5 \mathrm{v} 1.1 \mathrm{MS} / \mathrm{sec}$ 6.3fj/conversion-step SAR-ADC with tri-level comparator in 40nm CMOS. In VLSI Symposium on Circuits, pages 262 -263, June 2011.

[15] A. F. Yeknami. Low-Power Delta-Sigma Modulators for Medical Applications. PhD thesis, Linköping University, 2013.

[16] C.J.B. Fayomi and G.W. Roberts. Design and characterization of low-voltage analog switch without the need for clock boosting. In Midwest Symposium on Circuits and Systems (MWSCAS), volume 3, pages 315-318, July 2004.

[17] K. Roy, S. Mukhopadhyay, and H. Mahmoodi-Meimand. Leakage current mechanisms and leakage reduction techniques in deep-submicrometer CMOS circuits. Proceedings of the IEEE, 91(2):305 - 327, Feb. 2003.

[18] B.P. Ginsburg and A.P. Chandrakasan. 500-MS/s 5-bit ADC in 65-nm CMOS with split capacitor array DAC. IEEE Journal of Solid-State Circuits, 42(4):739 -747, April 2007.

[19] B. P. Ginsburg. Energy-Efficient Analog-to-Digital Conversion for UltraWideband Radio. PhD thesis, Massachusetts Institute of Technology, 2007. 
[20] A. Agnes, E. Bonizzoni, P. Malcovati, and F. Maloberti. A 9.4-ENOB 1V 3.8 $\mu \mathrm{W}$ $100 \mathrm{kS} / \mathrm{s}$ SAR ADC with time-domain comparator. In IEEE International SolidState Circuits Conference Digest of Technical Papers (ISSCC), pages 246-610, Feb. 2008.

[21] Yan Zhu, Chi-Hang Chan, U-Fat Chio, Sai-Weng Sin, Seng-Pan U, R. P. Martins, and F. Maloberti. A 10-bit 100-MS/s reference-free SAR ADC in $90 \mathrm{~nm}$ CMOS. IEEE Journal of Solid-State Circuits, 45(6):1111-1121, 2010.

[22] D. Zhang, A. Bhide, and A. Alvandpour. A 53-nW 9.1-ENOB 1-kS/s SAR ADC in 0.13- $\mu \mathrm{m}$ CMOS for medical implant devices. IEEE Journal of Solid-State Circuits, 47(7):1585 -1593, July 2012.

[23] Jieh-Tsorng Wu and B. A. Wooley. A 100-MHz pipelined CMOS comparator. IEEE Journal of Solid-State Circuits, 23(6):1379-1385, 1988.

[24] Rudy van de Plassche. CMOS Integrated Analog-to-Digital and Digital-toAnalog Converters. Kluwer Academic Publishers, 2003.

[25] Christer Svensson and J. Wikner. Power consumption of analog circuits: a tutorial. Analog Integrated Circuits and Signal Processing, 65:171-184, 2010.

[26] B. Razavi. Design of Analog CMOS Integrated Circuits. The McGraw-Hill, 2001.

[27] T. Kobayashi, K. Nogami, T. Shirotori, and Y. Fujimoto. A current-controlled latch sense amplifier and a static power-saving input buffer for low-power architecture. IEEE Journal of Solid-State Circuits, 28(4):523-527, 1993.

[28] P. Nuzzo, F. De Bernardinis, P. Terreni, and G. Van der Plas. Noise analysis of regenerative comparators for reconfigurable ADC architectures. IEEE Transactions on Circuits and Systems I: Regular Papers, 55(6):1441 -1454, July 2008.

[29] A. Nikoozadeh and B. Murmann. An analysis of latch comparator offset due to load capacitor mismatch. IEEE Transactions on Circuits and Systems II: Express Briefs, 53(12):1398 -1402, Dec. 2006.

[30] P. Nuzzo, G. Van der Plas, F. De Bernardinis, L. Van der Perre, B. Gyselinckx, and P. Terreni. A $10.6 \mathrm{~mW} / 0.8 \mathrm{pJ}$ power-scalable $1 \mathrm{GS} / \mathrm{s} 4 \mathrm{~b}$ ADC in $0.18 \mu \mathrm{m}$ CMOS with 5.8GHz ERBW. In 43rd ACM/IEEE Design Automation Conference (DAC), pages 873-878, 2006.

[31] J. Kim, B.S. Leibowitz, J. Ren, and C.J. Madden. Simulation and analysis of random decision errors in clocked comparators. IEEE Transactions on Circuits and Systems I: Regular Papers, 56(8):1844 -1857, Aug. 2009. 
[32] T. Sundstrom, B. Murmann, and C. Svensson. Power dissipation bounds for high-speed Nyquist analog-to-digital converters. IEEE Transactions on Circuits and Systems I: Regular Papers, 56(3):509 -518, March 2009.

[33] P.M. Figueiredo. Comparator metastability in the presence of noise. IEEE Transactions on Circuits and Systems I: Regular Papers, 60(5):1286-1299, 2013.

[34] C.L. Portmann and H.Y. Meng. Metastability in CMOS library elements in reduced supply and technology scaled applications. IEEE Journal of Solid-State Circuits, 30(1):39 -46, Jan. 1995.

[35] J.L. McCreary and P.R. Gray. All-MOS charge redistribution analog-to-digital conversion techniques - part I. IEEE Journal of Solid-State Circuits, 10(6):371 -379, Dec. 1975.

[36] M. Saberi, R. Lotfi, Khalil Mafinezhad, and W.A. Serdijn. Analysis of power consumption and linearity in capacitive Digital-to-Analog converters used in successive approximation ADCs. IEEE Transactions on Circuits and Systems I: Regular Papers, 58(8):1736-1748, 2011.

[37] K.-S. Tan, S. Kiriaki, M. De Wit, J.W. Fattaruso, C.-Y. Tsay, W.E. Matthews, and R.K. Hester. Error correction techniques for high-performance differential A/D converters. IEEE Journal of Solid-State Circuits, 25(6):1318-1327, 1990.

[38] J.A. McNeill, Ka Yan Chan, M.C.W. Coln, C.L. David, and C. Brenneman. All-digital background calibration of a successive approximation ADC using the split adc architecture. IEEE Transactions on Circuits and Systems I: Regular Papers, 58(10):2355-2365, 2011.

[39] Wenbo Liu, Pingli Huang, and Yun Chiu. A 12-bit, 45-MS/s, 3-mw redundant successive-approximation-register analog-to-digital converter with digital calibration. IEEE Journal of Solid-State Circuits, 46(11):2661-2672, 2011.

[40] D. A. Johns and K. Martin. Analog Integrated Circuit Design. John Wiley \& Sons, Inc., 1997.

[41] V. Stojanovic and V.G. Oklobdzija. Comparative analysis of master-slave latches and flip-flops for high-performance and low-power systems. IEEE Journal of Solid-State Circuits, 34(4):536-548, 1999.

[42] A.A. Abidi. High-frequency noise measurements on FET's with small dimensions. IEEE Transactions on Electron Devices, 33(11):1801-1805, 1986.

[43] C.H. Diaz, D.D. Tang, and J.Y.-C. Sun. CMOS technology for MS/RF SoC. IEEE Transactions on Electron Devices, 50(3):557-566, 2003. 
[44] Chih-Sheng Chang, Chih-Ping Chao, J.G.J. Chern, and J. Y C Sun. Advanced CMOS technology portfolio for RF IC applications. IEEE Transactions on Electron Devices, 52(7):1324-1334, 2005.

[45] L.S.Y. Wong, S. Hossain, A. Ta, J. Edvinsson, D.H. Rivas, and H. Naas. A very low-power CMOS mixed-signal IC for implantable pacemaker applications. IEEE Journal of Solid-State Circuits, 39(12):2446 - 2456, Dec. 2004.

[46] M. van Elzakker, E. van Tuijl, P. Geraedts, D. Schinkel, E. Klumperink, and B. Nauta. A $1.9 \mu \mathrm{W} 4.4 \mathrm{fJ} /$ conversion-step $10 \mathrm{~b} 1 \mathrm{MS} / \mathrm{s}$ charge-redistribution adc. In IEEE International Solid-State Circuits Conference Digest of Technical Papers (ISSCC), pages 244 -245, Feb. 2008.

[47] Chun-Cheng Liu, Soon-Jyh Chang, Guan-Ying Huang, and Ying-Zu Lin. A 10-bit 50-MS/s SAR ADC with a monotonic capacitor switching procedure. IEEE Journal of Solid-State Circuits, 45(4):731 -740, April 2010.

[48] K. Ishida, K. Kanda, A. Tamtrakarn, H. Kawaguchi, and T. Sakurai. Managing subthreshold leakage in charge-based analog circuits with low-VTH transistors by analog T-switch (AT-switch) and super cut-off CMOS (SCCMOS). IEEE Journal of Solid-State Circuits, 41(4):859 - 867, April 2006.

[49] G. Van der Plas and B. Verbruggen. A 150MS/s $133 \mu \mathrm{W} 7 \mathrm{~b}$ ADC in 90nm digital CMOS using a comparator-based asynchronous binary-search sub-ADC. In IEEE International Solid-State Circuits Conference Digest of Technical Papers (ISSCC), pages $242-244$, Feb. 2008.

[50] A Methodology for the Offset-Simulation of Comparators, [Online]. http: //www.designers-guide.org/Analysis/comparator.pdf.

[51] S.-W.M. Chen and R.W. Brodersen. A 6-bit 600-MS/s 5.3-mW asynchronous ADC in 0.13- $\mu \mathrm{m}$ CMOS. IEEE Journal of Solid-State Circuits, 41(12):2669 -2680 , Dec. 2006.

[52] A. Rossi and G. Fucili. Nonredundant successive approximation register for A/D converters. Electronics Letters, 32(12):1055 -1057, June 1996.

[53] S. Narendra, V. De, S. Borkar, D.A. Antoniadis, and A.P. Chandrakasan. Fullchip subthreshold leakage power prediction and reduction techniques for sub0.18- $\mu$ m CMOS. IEEE Journal of Solid-State Circuits, 39(3):501 - 510, March 2004.

[54] J. Doernberg, Hae-Seung Lee, and D. Hodges. Full-speed testing of A/D converters. IEEE Journal of Solid-State Circuits, 19(6):820-827, 1984. 
[55] D.C. Daly and A.P. Chandrakasan. A 6-bit, $0.2 \mathrm{~V}$ to $0.9 \mathrm{~V}$ highly digital flash ADC with comparator redundancy. IEEE Journal of Solid-State Circuits, 44(11):3030 -3038, Nov. 2009.

[56] J. Craninckx and G. Van der Plas. A 65fJ/conversion-step 0-to-50MS/s 0-to$0.7 \mathrm{~mW} 9 \mathrm{~b}$ charge-sharing SAR ADC in $90 \mathrm{~nm}$ digital CMOS. In IEEE International Solid-State Circuits Conference Digest of Technical Papers (ISSCC), pages $246-600$, Feb. 2007.

[57] Xiaodan Zou, Xiaoyuan Xu, Libin Yao, and Yong Lian. A 1-V 450-nW fully integrated programmable biomedical sensor interface chip. IEEE Journal of Solid-State Circuits, 44(4):1067 -1077, April 2009.

[58] J. Sauerbrey, D. Schmitt-Landsiedel, and R. Thewes. A 0.5-V 1- $\mu$ w successive approximation ADC. IEEE Journal of Solid-State Circuits, 38(7):1261 - 1265, July 2003.

[59] K. S. Tan. On board self-calibration of analog-to-digital and digital-to-analog converters, Aug. 1983.

[60] Hae-Seung Lee, D. Hodges, and P. R. Gray. A self-calibrating 15 bit CMOS A/D converter. IEEE Journal of Solid-State Circuits, 19(6):813-819, 1984.

[61] Christer Jansson. ADC calibration, Sep. 2012.

[62] Z. Boyacigiller, B. Weir, and P. Bradshaw. An error-correcting 14b/20us CMOS A/D converter. In IEEE International Solid-State Circuits Conference Digest of Technical Papers (ISSCC), volume XXIV, pages 62-63, 1981.

[63] R. K. Hester, K.-S. Tan, M. De Wit, J. W. Fattaruso, S. Kiriaki, and J. R. Hellums. Fully differential ADC with rail-to-rail common-mode range and nonlinear capacitor compensation. IEEE Journal of Solid-State Circuits, 25(1):173-183, 1990.

[64] P. E. Allen and D. R. Holberg. CMOS Analog circuit Design. Oxford University Press, 2nd edition, 2002.

[65] Thermal resistance - Wikipedia, [Online]. http://en.wikipedia.org/ wiki/Thermal_resistance.

[66] G.J. Hua. Non-binary capacitor array calibration for a high performance successive approximation analog-to-digital converter. $\mathrm{PhD}$ thesis, The University of Texas at Austin, 2003.

[67] Wenbo Liu and Yun Chiu. An equalization-based adaptive digital background calibration technique for successive approximation analog-to-digital converters. In Proc. ASICON, pages 289-292, 2007. 
[68] TI HealthTech Guide, [Online]. http://www.ti.com/lit/sg/ slyb108h/slyb108h.pdf.

[69] T. Morie, T. Miki, K. Matsukawa, Y. Bando, T. Okumoto, K. Obata, S. Sakiyama, and S. Dosho. A 71db-SNDR 50MS/s 4.2mW CMOS SAR ADC by SNR enhancement techniques utilizing noise. In IEEE International Solid-State Circuits Conference Digest of Technical Papers (ISSCC), pages 272-273, 2013.

[70] P. Harpe, E. Cantatore, and A. van Roermund. An oversampled 12/14b SAR ADC with noise reduction and linearity enhancements achieving up to $79.1 \mathrm{~dB}$ SNDR. In IEEE International Solid-State Circuits Conference Digest of Technical Papers (ISSCC), pages 194-195, 2014.

[71] E. Vittoz and J. Fellrath. CMOS analog integrated circuits based on weak inversion operations. IEEE Journal of Solid-State Circuits, 12(3):224-231, 1977.

[72] S. R. Norsworthy, R. Schreier, and G. C. Temes. Delta-Sigma Data Converters Theory, Design, and Simulation. John Wiley \& Sons, Inc., 1997.

[73] M. Dessouky and A. Kaiser. Input switch configuration suitable for rail-to-rail operation of switched op amp circuits. Electronics Letters, 35(1):8-10, 1999.

[74] C. P. Hurrell, C. Lyden, D. Laing, D. Hummerston, and M. Vickery. An 18 b 12.5 MS/s ADC with 93 dB SNR. IEEE Journal of Solid-State Circuits, 45(12):2647-2654, 2010.

[75] Wenbo Liu, Pingli Huang, and Yun Chiu. A 12b 22.5/45MS/s 3.0mW 0.059 $\mathrm{mm}^{2}$ CMOS SAR ADC achieving over 90dB SFDR. In IEEE International SolidState Circuits Conference Digest of Technical Papers (ISSCC), pages 380-381, 2010.

[76] R. Kapusta, Junhua Shen, S. Decker, Hongxing Li, and E. Ibaragi. A 14b 80MS/s SAR ADC with 73.6dB SNDR in 65nm CMOS. In IEEE International Solid-State Circuits Conference Digest of Technical Papers (ISSCC), pages 472-473, 2013.

[77] Guan-Ying Huang, Soon-Jyh Chang, Chun-Cheng Liu, and Ying-Zu Lin. A $1-\mu \mathrm{W} 10-$ bit $200-\mathrm{kS} / \mathrm{s}$ SAR ADC with a bypass window for biomedical applications. IEEE Journal of Solid-State Circuits, 47(11):2783-2795, 2012.

[78] F. M. Yaul and A. P. Chandrakasan. 11.3 A 10b 0.6nW SAR ADC with datadependent energy savings using LSB-first successive approximation. In IEEE International Solid-State Circuits Conference Digest of Technical Papers (ISSCC), pages 198-199, 2014. 
[79] J. A. Fredenburg and M. P. Flynn. A 90-MS/s 11-MHz-bandwidth 62-dB SNDR noise-shaping SAR ADC. IEEE Journal of Solid-State Circuits, 47(12):28982904, 2012.

[80] K. S. Kim, J. Kim, and S. H. Cho. nth-order multi-bit $\Sigma \Delta$ ADC using SAR quantiser. Electronics Letters, 46(19):1315-1316, 2010. 